Bruna Oneda

\title{
Efeitos isolados e associados da terapia de reposição oral estrogênica e do exercício físico aeróbio nas respostas hemodinâmicas e neurais em mulheres no período pós-menopausa
}

Tese apresentada à Faculdade de Medicina da Universidade de São Paulo para obtenção do título de Doutor em Ciências

Área de concentração: Nefrologia Orientadora: Tais Tinucci 


\section{Dados Internacionais de Catalogação na Publicação (CIP)}

Preparada pela Biblioteca da

Faculdade de Medicina da Universidade de São Paulo

\section{Creprodução autorizada pelo autor}

\section{Oneda, Bruna}

Efeitos isolados e associados da terapia de reposição oral estrogênica e do exercício físico aeróbico nas respostas hemodinâmicas e neurais em mulheres no período pósmenopausa / Bruna Oneda. -- São Paulo, 2010.

Tese(doutorado)--Faculdade de Medicina da Universidade de São Paulo.

Departamento de Clínica Médica.

Área de concentração: Nefrologia.

Orientadora: Tais Tinucci.

Descritores: 1.Pós-menopausa 2.Terapia de reposição hormonal 3.Terapia de reposição de estrogênios 4.Exercício 5.Sistema nervoso simpático 


\section{Dedicatória}

Dedico esse trabalho a meus pais, Sonia e Elaide, pelo amor incondicional e por serem meus exemplos nessa vida.

Às minhas irmãs Flávinha e Bia, por estarem do meu lado em todas as horas. 


\section{Agradecimentos}

Agradeço a todas as pessoas que contribuíram direto e indiretamente para a realização deste trabalho e, em especial:

À Taís Tinucci, minha orientadora, pela confiança, todo auxílio, incentivo e entusiasmo, mesmo nas horas mais difíceis.

Aos professores coordenadores do projeto Dr. Décio Mion Jr., Dra. Claudia Lúcia de Moraes Forjaz, Dra. Ângela Maggio da Fonseca e Dra Sandra B. Abrahão, pelos exemplos de pessoas e profissionais brilhantes.

À Fernanda Rocchi Bernardo que gentilmente cedeu seus dados e fez com que eu iniciasse esta etapa de meu trabalho.

Ao meu amigo Crivaldo Cardoso Jr., pela paciência, auxílio e exemplo de dedicação.

À minha amiga Josiane Lima de Gusmão, por estar presente em todas as horas alegres ou tristes dessa fase da minha vida e sempre com uma palavra de conforto.

À Tatiana Goveia Araújo, Silvana Barros, Maria Elisa e Aline Borjuca do Laboratório de Hipertensão pela ajuda e prontidão, em todos os momentos.

À Eliana Gonçalves e Pedro Henrique G Pereira da secretaria da pósgraduação.

Ao Marcelo Conteçote, pela correção ortográfica. 
A todas as voluntárias do estudo que viabilizaram a concretização do mesmo e me ensinaram muito sobre persistência e, principalmente, humildade.

Às pessoas que por não compreenderem a importância e significado deste processo na minha vida e, hoje não estão mais "ao meu lado", mas contribuíram enormemente para meu amadurecimento e fortalecimento.

$E$, finalmente, aos meus familiares e amigos que entenderam minhas ausências e torceram por mim e, mesmo distantes, permaneceram "sempre perto".

"Se procurar bem você acaba encontrando Não a explicação (duvidosa) da vida Mas a poesia (inexplicável) da vida" Carlos Drummond de Andrade 


\section{Sumário}

Lista de abreviaturas

Lista de figuras

Lista de tabelas

Resumo

Summary

1 INTRODUÇÃO............................................................... 1

1.1 Climatério e terapia hormonal.............................................. 1

1.2 Riscos e benefícios da terapia hormonal.................................... 3

1.3 Efeitos da terapia de estrogênica no sistema nervoso simpático e pressão arterial............................................................................... 7

1.4 Efeitos do exercício físico aeróbio nos sistema cardiovascular e nervoso autônomo da mulher na pós-menopausa............................... 10

1.5 Associação do exercício físico aeróbio e da terapia estrogênica........ 12

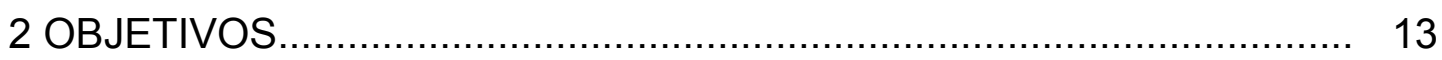

2.1 Gerais.................................................................................... 13

2.2 Específicos......................................................................... 13

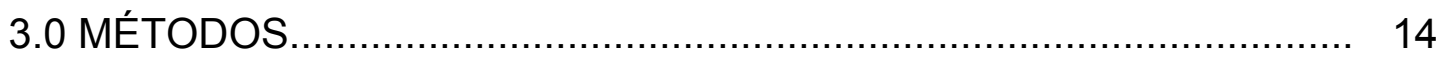

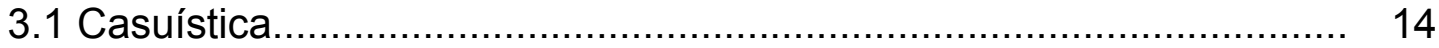

3.2 Critérios de inclusão ............................................................... 15

3.3 Protocolo experimental................................................... 15

3.3.1 Delineamento geral do estudo........................................... 15

$\begin{array}{ll}3.3 .2 \text { Sessões experimentais } & 17\end{array}$

3.4 Exames preliminares.......................................................... 19

3.4.1 Avaliação da menopausa...................................................... 19

3.4.2 Avaliação dos sintomas da menopausa...................................... 19

3.4.3 Avaliação de obesidade.......................................................... 19

3.4.4 Avaliação cardiovascular.................................................... 20

3.4.5 Avaliação de diabetes...................................................... 20

3.4.6 Avaliação de hipercolesterolemia.......................................... 20

3.4.7 Avaliação de hipertensão........................................................... 20 
3.4.8 Avaliação da capacidade aeróbia máxima................................ 21

3.5 Procedimentos experimentais............................................... 22

3.5.1 Terapia estrogênica (TRH) ou placebo (PLA)........................... 22

3.5.2 Treinamento físico (TF) ou sedentarismo (SED) ....................... 23

3.5.3 Exercícios estático e dinâmico com "handgrip"............................. 24

3.6 Medidas durante as sessões experimentais................................. 25

3.6.1 Pressão arterial e freqüência cardíaca...................................... 25

3.4.2 Fluxo sanguíneo do antebraço.............................................. 25

3.4.3 Atividade nervosa simpática periférica.................................. 26

3.7 Análise dos dados............................................................ 27

3.8 Análise estatística................................................................ 27

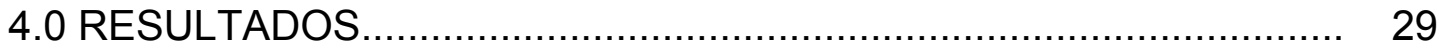

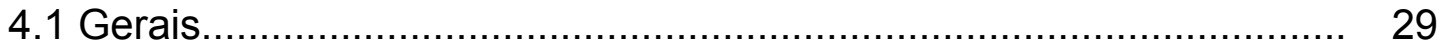

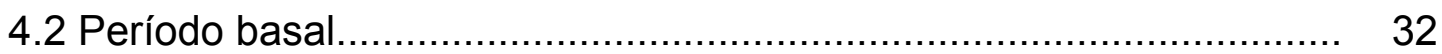

4.3 Exercícios estático e dinâmico com "handgrip"............................. 36

4.3.1 Atividade nervosa simpática periférica................................... 36

4.3.2 Pressão arterial sistólica................................................... 38

4.3.3 Pressão arterial diastólica...................................................... 41

4.3.4 Pressão arterial média.......................................................... 43

4.3.5 Frequência cardíaca............................................................ 46

4.3.6 Fluxo sanguíneo do antebraço............................................ 50

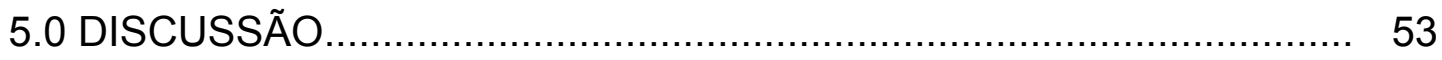

5.1 Período basal................................................................ 53

5.2 Exercícios estático e dinâmico com handgrip ............................. 58

5.3 Dificuldades e limitações do estudo................................................ 65

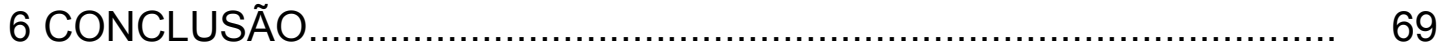

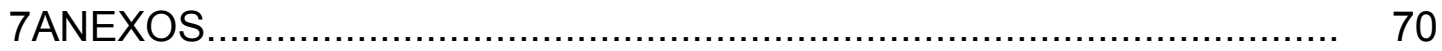

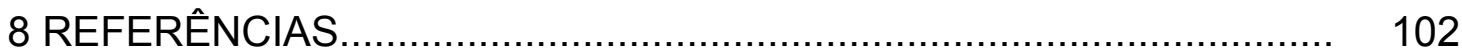




\section{LISTA DE ABREVIATURAS}

ANSP - Atividade nervosa simpática periférica

ED - Exercício dinâmico com "handgrip"

EE - Exercício estático com "handgrip"

FC - Freqüência cardíaca

CVM - Contração voluntária máxima

FSA - Fluxo sanguíneo do antebraço

FSH - Hormônio folículo estimulante

IMK - Índice menopausal de Kupperman

LH - Hormônio luteinizante

OCLU: Oclusão venosa

PAD - Pressão arterial diastólica

PAM - Pressão arterial média

PAS - Pressão arterial sistólica

PLA - Grupo placebo

RVP - Resistência vascular periférica

REC - Recuperação

REP - Repouso

SED - Grupo que permaneceu sedentário

TF - Grupo que realizou o treinamento físico aeróbio

TRH - Terapia de reposição estrogência/ grupo que recebeu TRH

$\mathrm{VO}_{2 \text { max }}$ - Consumo máximo de oxigênio 


\section{LISTA DE FIGURAS}

Figura 1 - Fluxograma geral do estudo 17

$\begin{array}{ll}\text { Figura } 2 \text { - Fluxograma das sessões experimentais } & 18\end{array}$

Figura 3 - Representação gráfica das respostas da ANSP dos grupos TF e SED nas sessões PRÉ e PÓS no período basal.......................................

Figura 4 - Representação gráfica das respostas do FSA dos grupos TF e

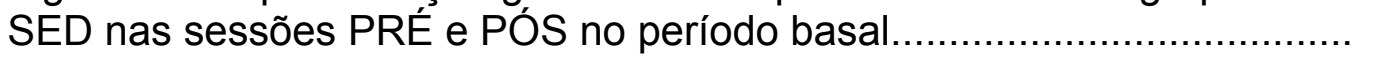

Figura 5 - Representação gráfica do comportamento da ANSP durante estágios do exercício estático e dinâmico com "handgrip" de todos os grupos, nas duas sessões.

Figura 6 - Representação gráfica do comportamento da PAS durante estágios do exercício estático e dinâmico com "handgrip" de todos os grupos, nas duas sessões

Figura 7 - Representação gráfica do comportamento da PAD durante estágios do exercício estático e dinâmico com "handgrip" de todos os grupos, nas duas sessões.

Figura 8 - Representação gráfica do comportamento da PAM durante estágios do exercício estático e dinâmico com "handgrip" de todos os grupos, nas duas sessões.

Figura 9 - Representação gráfica das respostas de $\mathrm{FC}(\mathrm{bpm})$ durante os estágios do exercício estático com "handgrip" dos grupos SED-PLA e SED-TRH.

Figura 10 - Representação gráfica das respostas de FC (bpm) durante os estágios do exercício estático com "handgrip" dos grupos TF-PLA e TF-TRH

Figura 11 - Representação gráfica das respostas de FC (bpm) durante estágios do exercício dinâmico com "handgrip" dos grupos PLA e TRH nas sessões PÓS.

Figura 12 - Representação gráfica do comportamento da FC durante estágios do exercício estático e dinâmico com "handgrip" de todos os grupos, nas duas sessões.

Figura 13 - Representação gráfica do comportamento do FSA durante estágios do exercício estático e dinâmico com "handgrip" de todos os grupos, nas duas sessões. 


\section{LISTA DE TABELAS}

Tabela 1 - Características da amostra

Tabela 2 - Médias dos valores dos exames laboratoriais no início (PRÉ) e após 6 meses de estudo (PÓS).

Tabela 3 - Variáveis analisadas no estudo no período basal, divididas por grupo nas sessões PRÉ e PÓS

Tabela 4 - Respostas de ANSP (impulsos/min) dos grupos durante os estágios do exercício estático com "handgrip" nas sessões PRÉ e PÓS...

Tabela 5 - Respostas de ANSP (impulsos/min) dos grupos durante os estágios do exercício dinâmico com "handgrip" nas sessões PRÉ e PÓS.

Tabela 6 - Respostas de PAS $(\mathrm{mm} \mathrm{Hg})$ dos grupos durante os estágios do exercício estático com "handgrip" nas sessões PRÉ e PÓs

Tabela 7 - Respostas de PAS ( $\mathrm{mm} \mathrm{Hg}$ ) dos grupos durante os estágios do exercício dinâmico com "handgrip" nas sessões PRÉ e PÓS.

Tabela 8 - Respostas de PAD ( $\mathrm{mm} \mathrm{Hg}$ ) dos grupos durante os estágios do exercício estático com "handgrip" nas sessões PRÉ e PÓS

Tabela 9 - Respostas de PAD ( $\mathrm{mm} \mathrm{Hg}$ ) dos grupos durante os estágios do exercício dinâmico com "handgrip" nas sessões PRÉ e PÓS.

Tabela 10 - Respostas de PAM (mm Hg) dos grupos durante os estágios do exercício estático com "handgrip" nas sessões PRÉ e PÓS

Tabela 11 - Respostas de PAM (mm Hg) dos grupos durante os estágios do exercício dinâmico com "handgrip" nas sessões PRÉ e PÓS

Tabela 12 - Respostas de FC (bpm) dos grupos durante os estágios do exercício estático com "handgrip" nas sessões PRÉ e PÓS

Tabela 13 - Respostas de FC (bpm) dos grupos durante os estágios do exercício dinâmico com "handgrip" nas sessões PRÉ e PÓS.

Tabela 14 - Respostas de FSA [ml(min.100ml)] dos grupos durante os 50 estágios do exercício estático com "handgrip" nas sessões PRÉ e PÓS...

Tabela 15 - Respostas de FSA [ml(.min.100ml)] dos grupos durante os estágios do exercício dinâmico com "handgrip" nas sessões PRÉ e PÓS. 


\section{RESUMO}

Oneda B. Efeitos isolados e associados da terapia de reposição oral estrogênica do exercício físico aeróbio nas respostas hemodinâmicas e neurais em mulheres no período pós-menopausa [tese]. São Paulo: Faculdade de Medicina, Universidade de São Paulo; 2010.

A pós-menopausa é marcada por alterações fisiológicas hemodinâmicas e metabólicas. A terapia de reposição estrogênica é uma forma de amenizar as conseqüências da deficiência hormonal e o exercício físico contribui significativamente para a redução do risco cardiovascular. $\mathrm{O}$ objetivo desse estudo foi avaliar em mulheres pós-menopausadas os efeitos isolados e associados da terapia oral estrogênica (TRH) e do treinamento físico aeróbio (TF) nas respostas hemodinâmicas e neurais basais e durante os exercícios

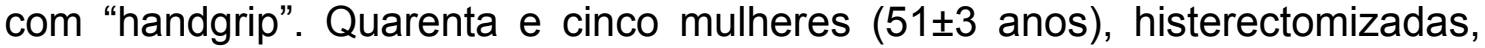
com e sem ovários, saudáveis, realizaram uma sessão experimental e, posteriormente foram divididas em 4 grupos SED-PLA $(n=11)$, SED-TRH $(n=14)$, TF-PLA $(n=12)$ e TF-TRH $(n=8)$. Os grupos TRH e receberam valerato de estradiol $1 \mathrm{mg} / \mathrm{dia}$; PLA receberam placebo; TF, realizaram exercício aeróbio em cicloergômetro por 50 minutos, 3 vezes por semana e SED permaneceram sedentárias. Todas as voluntárias participaram de uma segunda sessão experimental após 6 meses de acompanhamento. Nas sessões experimentais foram avaliadas a atividade nervosa simpática periférica (ANSP microneurografia), pressão arterial, freqüência cardíaca (FC - método oscilométrico - Dixtal no membro inferior), fluxo sangüíneo do antebraço (FSA pletismografia) em um período basal e durante exercícios estático e dinâmico com "handgrip" a $30 \%$ da força de contração máxima. Para análise estatística foi utilizada ANOVA. O TF isoladamente diminuiu ANSP de $40 \pm 7$ a $34 \pm 4$ impulsos/min, $\quad(P=0,01)$ e aumentou FSA de $1,92 \pm 0,96$ a $2,65 \pm 1,34$ $\mathrm{ml}(\min .100 \mathrm{ml}), \mathrm{P}=0,03$ no período basal. TRH e TF associados reduziram a FC no período basal de $65 \pm 8$ para $62 \pm 7 \mathrm{bpm}(\mathrm{P}=0,01)$ e durante o exercício estático e dinâmico com "handgrip". A TRH de maneira isolada ou associada ao TF diminuiu as respostas de FC durante os exercícios estático e dinâmico com "handgrip. Em conclusão, as intervenções de maneira isolada ou associada promovem alterações hemodinâmicas e neurais que podem contribuir para redução do risco cardiovascular de mulheres pós-menopausadas saudáveis.

Descritores: Pós-menopausa, terapia de reposição hormonal, terapia de reposição de estrogênios, exercício, sistema nervoso simpático 


\section{SUMMARY}

Oneda B. Effects of estrogen replacement therapy in hemodinamic and neural responses to acute aerobic exercise in post-menopausal women. [thesis]. São Paulo: “Faculdade de Medicina, Universidade de São Paulo”; 2010.

The post-menopause is marked by physiological hemodynamic and metabolic changes. The estrogen replacement therapy is a way to reduce the consequences of hormone deficiency and physical exercise contributes significantly to the reduction of cardiovascular risk. The aim of this study was to evaluate in post-menopausal women the isolated and associated effects of oral estrogen therapy (TRH) and physical training (TF) in the neural and hemodynamic responses during baseline and "handgrip" exercises. Forty-five women (51 \pm 3 years), hysterectomized, with or without ovaries, healthy, participated of an initial session and then they were divided into 4 groups SEDPLA $(n=11)$, SED-TRH $(n=14)$, TF-PLA $(n=12)$ and TF-TRH $(n=8)$. The TRH groups received estradiol valerate $1 \mathrm{mg} /$ day; PLA placebo; TF, performed aerobic exercise on a cycle ergometer for 50 minutes, 3 times a week and SED remained sedentary. All subjects participated in a second experimental session after 6 months of follow-up. In the experimental sessions peripheral sympathetic nerve activity (ANSP - microneurography), blood pressure, heart rate (FC oscillometry - Dixtal lower limb), forearm blood flow (FSA - plethysmography) were evaluated at the baseline period and during static and dynamic "handgrip" exercises at $30 \%$ of the maximum force. ANOVA was used for the statistica analysis. The TF alone decreased ANSP from $40 \pm 7$ to $34 \pm 4$ bursts $/ \mathrm{min}, \mathrm{P}=$ 0.01 and increased FSA $1.92 \pm 0.96$ to $2.65 \pm 1.34 \mathrm{ml}(\min .100 \mathrm{ml}), P=0.03$ at the baseline. The association of TRH and TF reduced HR at the baseline from $65 \pm 8$ to $62 \pm 7 \mathrm{bpm}(P=0.01)$ and during exercise with static and dynamic "handgrip". HRT alone or associated with TF decreased the HR responses during static and dynamic "handgrip exercises. In conclusion, the interventions alone or in an associated way promote neural and hemodynamic changes that may contribute to cardiovascular risk reduction in healthy postmenopausal women.

Descriptors: Postmenopause, hormone replacement therapy, estrogen replacement therapy, exercise, sympathetic nerve system 


\section{INTRODUÇÃO}

\subsection{Climatério e terapia hormonal}

As mulheres no período entre os 40 e 50 anos de idade começam a sofrer irregularidades no ciclo menstrual e falha na ovulação devido à diminuição da produção ovariana de $17 \beta$-estradiol e como mecanismo compensatório, ocorre uma elevação do nível sérico do hormônio folículo estimulante (FSH) e também do hormônio luteinizante $(\mathrm{LH})^{1}$. Esse período é denominado climatério e o último ciclo menstrual é chamado de menopausa ${ }^{2}$. As alterações hormonais resultam em alguns sintomas que são de grande importância diagnóstica. Os sintomas vasomotores afetam cerca de 60 a $80 \%$ das mulheres na pós-menopausa e pode ser experienciado por $90 \%$ delas na peri-menopausa ${ }^{3}$. A Peri-menopausa é caracterizada pelo intervalo de um a dois anos antes da menopausa que as irregularidades mentruais são comuns, e um ano após, quando ainda pode ocorrer novo fluxo ${ }^{4}$. Com o passar do tempo e o aumento do hipoestrogenismo ocorre hipotrofia das mamas e do aparelho urogenital, alterações na pele e aceleração na perda da massa óssea. A redução na massa óssea é responsável pelo aparecimento da osteoporose pósmenopausal, que pode resultar em fraturas ósseas e suas conseqüências danosas para a mulher ${ }^{2}$. As modificações fisiológicas, metabólicas e endócrinas causadas pela deficiência do estrogênio culminam com a predisposição do surgimento ou agravamento de diversas doenças, 
sobretudo as de ordem cardiovascular ${ }^{5}$. Sabe-se que a deficiência estrogênica provoca aumento do colesterol total e dos triglicérides, além do aumento das lipoproteínas de baixa densidade (LDL) e diminuição das lipoproteínas de alta densidade $(\mathrm{HDL})^{6}$. Observa-se também o acúmulo de gordura na região visceral, diminuição da secreção e da sensibilidade do organismo à ação da insulina e a hiperinsulinemia compensatória ${ }^{6}$. Além das modificações metabólicas, há aumento da reabsorção renal de sódio ${ }^{7}$, aumento na atividade nervosa simpática e alterações no leito vascular que contribuem para a elevação da pressão arterial ${ }^{6,8,9}$.

A utilização crônica do estrogênio, também conhecida como terapia de reposição estrogênica, surgiu com o objetivo de tratar e prevenir os sinais e sintomas do climatério².

A reposição hormonal pode ser feita pela via oral ou pela via não-oral. A via oral utiliza comprimidos contendo estrogênio ou estrogênio / progestagênio que são absorvidos e metabolizados pelo intestino antes de caírem na circulação sistêmica. A via não-oral ou parenteral inclui a transdérmica (na apresentação de adesivos ou gel), implantes subcutâneos, nasal, vaginal e intra-uterina (DIU) ${ }^{10}$

De acordo com Fonseca et al., (2002) ${ }^{2}$, os esquemas terapêuticos mais empregados são:

a) Contínuo simples: quando se utiliza apenas um hormônio, estrogênio ou progestagênio de forma contínua;

b) Combinado contínuo: quando se empregam doses fixas de progestagênio e estrogênio associados; 
c) Cíclico simples: administra-se de forma cíclica apenas um hormônio, estrogênio ou progestagênio;

d) Combinado cíclico contínuo: quando se utiliza estrogênio de forma contínua e progestagênio de forma cíclica;

e) Combinado cíclico seqüencial: administração do estrogênio durante 21 a 25 dias e nos últimos 10 a 12 dias associa-se progestagênio, simulando a fisiologia menstrual.

Dessa forma, com o objetivo principal de aliviar os sintomas que incomodam as mulheres e contribuir para a saúde da mulher no climatério, a terapia hormonal surgiu como principal forma de tratamento ${ }^{2}$, no entanto, sua utilização deve ser feita de forma individualizada e com muita cautela, considerando seus riscos e benefícios.

\subsection{Riscos e benefícios da terapia hormonal}

Diversos estudos têm sido conduzidos nos últimos 20 anos no intuito de investigar os efeitos da terapia estrogênica nos sintomas e na saúde da mulher no climatério. Um dos principais sintomas vasomotores deste período são os fogachos que são caracterizados por intenso calor que se inicia no tórax e aumenta em direção ao pescoço e face ${ }^{11}$. Os fogachos parecem atingir cerca de $75 \%$ das mulheres caucasianas ${ }^{12}$ e para a Sociedade Norte Americana da Menopausa (NAMS) os sintomas vasomotores são a indicação primária para administração hormonal ${ }^{13}$. Utian et al., (2001), ${ }^{14}$ verificaram redução dos sintomas vasomotores independentemente da dose 
de estrogênio associada ao progestagênio. Porém, doses mais altas do estrogênio isolado reduziram estes sintomas mais efetivamente.

No que diz respeito ao sistema cardiovascular, um estudo que acompanhou 121.700 enfermeiras verificou diminuição de doença arterial coronariana e mortalidade nas mulheres que faziam uso de terapia estrogênica ${ }^{15}$. Esse resultado foi um grande incentivo para a realização de outras pesquisas para avaliar a ação do estrogênio na redução do risco cardiovascular, porém os estudos seguintes não obtiveram resultados positivos. O "Heart disease and estrogen replacement study" (HERS) $)^{16}$ foi o primeiro estudo que visava avaliar os efeitos do hormônio na prevenção secundária de doença coronariana. Ele acompanhou 2.763 mulheres que receberam estrogênio equino conjugado ou placebo e mostrou aumento do risco de eventos cardiovasculares durante o primeiro ano da terapia. Uma grande crítica à metodologia desse estudo foi que a média de idade das participantes era muito avançada (67 anos), portanto com longo tempo da pós-menopausa ${ }^{17}$.

Outro protocolo publicado posteriormente foi o $\mathrm{ESPRIT}^{18}$ que utilizou $2 \mathrm{mg} /$ dia de esrogênio a partir de 30 dias após primeiro infarto do miocárdio em mulheres de 50 a 69 anos e também não verificou benefício do uso do hormônio. Em seguida, Viscoli et al., $(2001)^{19}$ administraram dose de $1 \mathrm{mg} /$ dia de $17 ß$ estradiol em 664 mulheres pós-menopausadas que já haviam sofrido isquemia cardíaca e verificaram após 2,8 anos que o hormônio não diminuía os riscos de reincidência dos eventos e ainda, aumentava o risco de derrame fatal. 
Os protocolos que objetivaram avaliar os efeitos do estrogênio na prevenção cardiovascular primária também não obtiveram resultados positivos e o estudo "Women's Health Initiative" (WHI) com a intenção de acompanhar 2 grupos de mulheres por 8,4 anos, apesar de inúmeras limitações em sua metodologia, não demonstrou benefícios da terapia hormonal no que diz respeito ao sistema cardiovascular. Um dos grupos foi composto por 16.608 mulheres com útero intacto que utilizaram estrogênio equino conjugado $(0,625 \mathrm{mg} / \mathrm{dia})$ associado a $2,5 \mathrm{mg} / \mathrm{dia}$ de acetato de medroxiprogestagênio ${ }^{20}$ e outro grupo composto por 10.739 mulheres histerectomizadas que utilizaram $0,625 \mathrm{mg} / \mathrm{dia}$ de estrogênio eqüino conjugado versus placebo ${ }^{21}$. $\mathrm{O}$ braço do estudo que associou o estrogênio à progestagênio foi interrompido após 5,2 anos, pois o índice global de riscos superou os benefícios sendo encontrada elevada ocorrência de doença arterial coronariana, derrame cerebral e embolismo pulmonar. $\mathrm{O}$ braço do estudo que avaliou a ação do estrogênio isolado foi interrompido com 6,8 anos devido ao elevado risco de derrame cerebral e a inexistência de benefícios para doença coronariana ${ }^{21}$. Assim como no estudo HERS, a metodologia do estudo WHI foi amplamente criticada, principalmente no que diz respeito à idade das pacientes envolvidas no estudo, que foi de 68,5 anos. Além disso, o medicamento não foi administrado nos primeiros anos após a menopausa o que contrasta com uma situação clínica normal ${ }^{17}$.

Apesar das críticas, os estudos serviram como base para que recomendações e diretrizes sobre sua utilização fossem criadas e a terapia estrogênica não mais foi utilizada como forma de prevenção primária ou 
secundária de doenças cardiovasculares ${ }^{22}$. As pesquisas também contribuíram para o despertar a consciência para o uso da terapia com bastante cautela ${ }^{13}$.

Além dos achados relacionados ao risco cardiovascular evidenciados pelo $\mathrm{WHI}$, este estudo demonstrou que o estrogênio associado à progestagênio aumentou o risco de câncer de mama, fato que não ocorreu em usuárias do estrogênio isolado ${ }^{20,21}$. O "Million Women Study"23 publicado em 2003 com 1.084.110 mulheres do Reino Unido, verificou que as duas formas de utilização do estrogênio elevaram o risco de câncer de mama e que o risco aumenta quanto maior o tempo de utilização da terapia. Neste protocolo, o risco só não foi aumentado nas usuárias de estrogênio isolado que na data de entrada do estudo usavam o hormônio há menos de um ano.

O principal benefício encontrado nos estudos do WHI foi a diminuição do risco de fratura de quadril com utilização das duas formas de terapia ${ }^{20,21}$. O estrogênio associado à progestagênio também diminuiu o risco de câncer colo-retal, fato não observado no uso da terapia estrogênica isolada.

A agência europeia para avaliação de produtos médicos (EMEA) recomenda: "a dose mínima e efetiva de hormônio durante o período mais curto independentemente da fórmula" ${ }^{24}$. Apesar dessa definição não ser universalmente aceita são consideradas baixas doses de estrogênio: $1 \mathrm{mg} /$ dia de estradiol, $0,312 \mathrm{mg} /$ dia de estrogênio equino conjugado, 0,025 $\mathrm{mg} / \mathrm{dia}$ de um adesivo de estrogênio ou uma dose de $0,75 \mathrm{mg} / \mathrm{dia}$ de estradiol gel administrado transdermicamente ${ }^{25}$. 
Dessa forma, ponderando os riscos e benefícios da terapia hormonal, a Sociedade Norte Americana da Menopausa recomenda que o uso da terapia seja consistente com os objetivos do tratamento e que os e riscos e benefícios sejam avaliados individualmente, considerando causa da menopausa, tempo desde a menopausa, sintomas e aspectos (como sexualidade e sono) que podem impactar na qualidade de vida além dos riscos de doença coronariana, derrame cerebral, diabetes melitus e outras condições $^{13}$. Além disso, devem ser considerados o fator idade e a via de administração do hormônio ${ }^{25}$.

\subsection{Efeitos da terapia estrogênica no sistema nervoso simpático e} pressão arterial

O sistema nervoso simpático é um importante regulador metabólico e hemodinâmico, local e sistêmico ${ }^{26}$. A atividade nervosa simpática aumentada eleva a pressão arterial e contribuí para o desenvolvimento e manutenção da hipertensão arterial sistêmica pela estimulação do coração, vasos periféricos, rins, causando aumento no débito cardíaco, resistência vascular e retenção hídrica ${ }^{27}$. Além disso, falha no balanço autonômico (aumento no tônus simpático acompanhado de redução do tônus parassimpático) tem sido associada a varias anormalidades metabólicas, hemodinâmicas, que resultam no aumento da morbidade e mortalidade cardiovascular ${ }^{28}$. O controle neural do aparelho circulatório pode ser avaliado em humanos por meio de dosagens de neuro-transmissores, pelo registro intraneural da atividade nervosa simpática periférica (ANSP) para o músculo 
(microneurografia), e pela análise espectral da frequência cardíaca ${ }^{29}$. Estudos que utilizaram a técnica de microneurografia verificaram ANSP aumentada em indivíduos com hipertensão essencial ${ }^{30,}{ }^{31}$. Alem disso, estudos evidenciaram que há diferença na atividade simpática entre os gêneros e modificações com o envelhecimento ${ }^{32,33}$. Até a menopausa, a ANSP em repouso ${ }^{32}$ é menor nas mulheres do que nos homens de mesma idade. Após este evento, no entanto, ocorre um grande aumento do efluxo nervoso simpático das mulheres, que acaba sendo comparável ao dos homens da mesma faixa etária e maior do que mulheres no período prémenopausa de mesma idade ${ }^{34}$.

Um estudo que utilizou estrogênio oral e transdérmico por oito semanas verificou que esta última forma de administração reduziu em $30 \%$ a atividade simpática em mulheres pós-menopausadas ${ }^{8}$.

Com relação à administração oral do estrogênio, não foram observadas alterações na ANSP de mulheres pós-menopausadas após seis meses de tratamento com estrogênio e uma semana de progestagênio nos meses 3 e 6 do estudo ${ }^{35}$.

Acredita-se, então, que os esteróides gonadais influenciem de alguma forma a atividade nervosa simpática. No entanto, as discrepâncias nos resultados dos estudos parecem estar relacionadas ao tipo, às diferentes vias de administração dos hormônios, bem como ao tempo de tratamento. Portanto, ainda não estão claros os efeitos do estrogênio na função autonômica ${ }^{36}$. 
A hipertensão arterial, considerada principal fator de risco cardiovascular, até aproximadamente os 45 anos atinge uma maior porcentagem de homens ${ }^{37}$ porém, entre os 45 e 54 anos acomete uma porcentagem semelhante em ambos os sexos. A partir dos 55 anos de idade, as mulheres apresentam uma incidência maior de hipertensão arterial do que os homens $(51 \% \text { vs } 45 \%)^{37}$. Acredita-se que esse aumento relacionase ao processo natural do envelhecimento ${ }^{38}$ e também pode ser influenciado pela deficiência estrogênica ${ }^{39}$.

Não existe, no entanto, um consenso sobre influência da terapia hormonal na pressão arterial. Existem inúmeros estudos indicando que a terapia hormonal reduz ${ }^{40,41}$, eleva ${ }^{42,43}$ ou não afeta ${ }^{44-46}$ a pressão arterial em mulheres normotensas. $O$ que se sabe até o momento é que o estrogênio exerce algumas ações benéficas no leito vascular ${ }^{47}$. Sua ação direta promove vasodilatação aguda, aumentando a síntese e bioatividade do óxido nítrico ${ }^{48} \mathrm{e}$ a longo prazo regula produção da óxido nítrico sintase ${ }^{49}$. Em humanos, foi verificado aumento nos níveis séricos de óxido nítrico após utilizar diversas formas de terapia de reposição hormonal por 12 meses ${ }^{50}$. Esses resultados, portanto podem influenciar a diminuição da pressão arterial. Além disso, sabe-se que o estrogênio inibe a vasoconstrição induzida pela endotelina ${ }^{51}$.

O tipo e as formas de administração de hormônio que produzem os maiores efeitos no leito vascular ainda precisam de mais esclarecimentos. 


\subsection{Efeitos do exercício físico aeróbio nos sistemas cardiovascular} e nervoso autônomo da mulher na pós-menopausa

A eficiência dos exercícios físicos na prevenção de risco cardiovascular já é comprovada pela literatura e por isso eles são amplamente recomendados ${ }^{52}$. O exercício aeróbio (dinâmico) é o tipo de exercício que produz sabidamente maior benefício cardiovascular. Segundo o "American College of Sports Medicine"53, esses exercícios são caracterizados por atividades realizadas com movimentos cíclicos, envolvendo grandes grupos musculares, com intensidade leve a moderada (50\% a $80 \%$ do $\mathrm{VO}_{2}$ máx.) e com longa duração (de 20 a 60 minutos).

Uma única sessão desses exercícios (exercício agudo) pode reduzir a

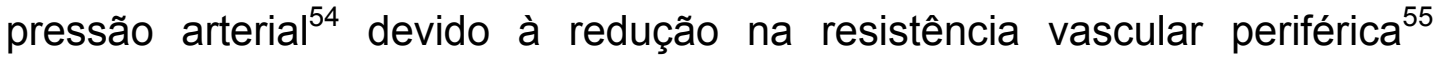
promovida pela atenuação da atividade nervosa simpática periférica ${ }^{56}$. Sabese que esses resultados podem ser potencializados quando os exercícios são feitos periodicamente, isto é, com periodicidade regular. Estudos envolvendo exercícios em mulheres pós-menopausada ainda são escassos, porém uma revisão sobre o assunto encontrou 28 estudos que o abordavam e os resultados foram unânimes confirmando que um programa de exercícios melhora o condicionamento cardiovascular (melhora no $\mathrm{VO}_{2}$ máximo entre 4 e 32\% com 30-65 minutos de exercícios entre 2 e 5 dias da semana), aumenta a força muscular, contribui para redução de peso, melhora a massa óssea e ainda reduz os níveis de pressão arterial ${ }^{57}$. Sabese também que os exercícios físicos regulares melhoram a qualidade de vida da mulher pós-menopausada ${ }^{58}$. 
Mais de sete mil pacientes que participaram do estudo WHI entre 50 a 79 anos e realizavam pelo menos 2,5 horas de caminhadas rápidas na semana tiveram $30 \%$ de redução de eventos cardiovasculares em 3,2 anos de acompanhamento do estudo. O "Estudo das enfermeiras" que acompanhou 72488 mulheres por 8 anos que realizavam tanto 3 horas semanais de caminhada rápida quanto 1 hora e meia de exercícios vigorosos tiveram 30 a $40 \%$ de redução nas taxas de infarto no miocárdio comparado às mulheres sedentárias ${ }^{59}$. No WHI as mulheres que caminhavam no mínimo de 1 hora na semana tiveram redução de $50 \%$ no risco de doença cardíaca comparado à mulheres que não praticavam atividades físicas vigorosas ${ }^{60}$.

No que diz respeito à pressão arterial, um estudo observou que caminhada por 5 dias em 15 semanas por 30-60 minutos a $65 \%$ do $\mathrm{VO}_{2}$ máximo reduziu $3 \mathrm{~mm} \mathrm{Hg}$ na pressão arterial diastólica, porém caminhadas em intensidades de 55 ou $45 \%$ do $\mathrm{VO}_{2}$ máximo não verificaram efeitos na pressão arterial $^{61}$. Outros estudos com caminhada ${ }^{62,63}$ ou que associaram caminhada a algum outro tipo de exercício aeróbio ${ }^{64}$ entre 30-60 minutos não promoveram mudanças na pressão arterial.

Dessa forma, sabe-se que os exercícios são benéficos ao sistema cardiovascular, mas ainda não há um consenso sobre seus reais efeitos nas mulheres pós-menopausadas. 


\subsection{Associação do exercício físico aeróbio e da terapia estrogênica}

A associação dos exercícios físicos com a terapia estrogênica foi muito pouco investigada até o momento. No que diz respeito ao condicionamento físico, o estrogênio parece não influenciar a capacidade aeróbia de mulheres pós-menopausadas. Foi o que observou um estudo que utilizou terapia hormonal combinada após a realização de 2 semanas de exercícios ( 5 vezes por semana) em bicicleta ergométrica ${ }^{65}$. Snabes et al. ${ }^{66}$ também não verificaram melhora no consumo máximo de oxigênio e nem no tempo total de exercício após 3 meses de terapia estrogênica (estradiol micronizado, $2 \mathrm{mg} / \mathrm{dia})$. Mulheres com doença coronariana que realizaram exercício aeróbico entre 75 e $80 \%$ do $\mathrm{VO}_{2}$ máximo, por 5 dias por 26 semanas não sofreram influência na melhora da capacidade aeróbia com uso de terapia hormonal (estrogênio ou estrogênio associado ao progestagênio) ${ }^{67}$.

Um estudo de nosso laboratório não observou efeito da terapia estrogênica (1mg/dia) nas respostas de pressão arterial, fluxo sanguíneo do antebraço e atividade nervosa simpática periférica após uma sessão de exercício aeróbio em bicicleta ergométrica ${ }^{68}$. A avaliação dessas variáveis hemodinâmicas e neurais na associação da terapia estrogênica e exercício crônico ainda não foram estudadas. 


\title{
2 OBJETIVOS
}

\begin{abstract}
2.1 Gerais
Avaliar e comparar os efeitos isolados e associados da reposição hormonal oral estrogênica e do exercício físico aeróbio em mulheres no período da pós-menopausa.
\end{abstract}

\subsection{Específicos}

Avaliar e comparar os efeitos isolados e associados da reposição hormonal oral estrogênica e do exercício físico aeróbio em mulheres no período da pós-menopausa:

1- nos valores basais de atividade nervosa simpática periférica, fluxo sangüíneo do antebraço, pressão arterial e freqüência cardíaca;

2- nas respostas neurais e hemodinâmicas ao exercício físico estático e dinâmico com "handgrip" a 30\% da contração voluntária máxima. 


\section{MÉTODOS}

\subsection{Casuística}

Foram estudadas 45 voluntárias, na faixa etária entre 45 e 60 anos, histerectomizadas, com e sem ovários, não obesas, não fumantes, normocolesterolêmicas e normotensas na admissão, e que não realizavam atividades físicas regularmente. A pacientes deveriam ser histerectomizadas, pois o estrogênio isolado (medicamento utilizado no estudo) não é recomendado para mulheres que possuem útero. Foram excluídas, as voluntárias que apresentaram problemas cardíacos, diagnóstico de câncer de mama ou de ovários e que eram hipersensíveis às drogas utilizadas no estudo.

A casuística foi selecionada dentre as mulheres atendidas pelo ambulatório de Ginecologia do Hospital das Clínicas da Faculdade de Medicina da Universidade de São Paulo. Esse protocolo é parte de um estudo temático que foi submetido e aprovado pelo Comitê de Ética do Hospital das Clínicas da Faculdade de Medicina da Universidade de São Paulo, auxílio FAPESP ( $\left.n^{\circ} 01 / 14989-7\right)$ (Anexo A).

Todas as voluntárias, antes de iniciarem os experimentos, foram esclarecidas, em uma entrevista, a respeito de todos os procedimentos experimentais, riscos e benefícios envolvidos no estudo e assinaram o Termo de Consentimento Livre e Esclarecido (Anexo B). 


\subsection{Critérios de inclusão}

A partir da realização dos exames preliminares, foram incluídas no estudo:

- mulheres de idade entre 45 e 60 anos,

- histerectomizadas, como ou sem ovários,

- na pós-menopausa;

- não obesas;

- sem problemas cardíacos;

- não diabéticas;

- não hipercolesterolêmicas;

- normotensas

\subsection{Protocolo experimental}

\subsubsection{Delineamento geral do estudo}

Inicialmente, as voluntárias selecionadas submeteram-se aos exames preliminares descritos posteriormente (item 3.4) e foram excluídas aquelas que não se enquadraram nos critérios de inclusão do estudo (item 3.2). Na seqüência, todas as voluntárias foram encaminhadas para a realização da sessão experimental - sessão PRÉ. Após a realização dessa sessão, a alocação das voluntárias foi realizada de forma aleatória nos quatro grupos experimentais: SED-PLA (sedentário + placebo), SED-TRH (sedentário + terapia hormonal estrogênica), TF-PLA (treinamento físico + placebo), TF- 
TRH (treinamento físico + terapia hormonal estrogênica). A alocação da medicação foi duplo-cega, ou seja, nem a voluntária, nem os pesquisadores tinham conhecimento de qual medicação cada voluntária estava usando.

Durante o estudo, todas as voluntárias foram acompanhadas a cada dois meses em consultas clínicas. Além disso, as voluntárias que compuserem os grupos TF-PLA e TF-TRH participaram do protocolo de treinamento físico conforme descrito no item 3.5.2.

Após seis meses de estudo, todas as voluntárias foram reavaliadas sessão PÓS. Nessa reavaliação foram repetidos os exames preliminares (item 3.3) e a sessão experimental.

A Figura 1 ilustra o delineamento geral do estudo. 
Figura 1 - Fluxograma geral do estudo

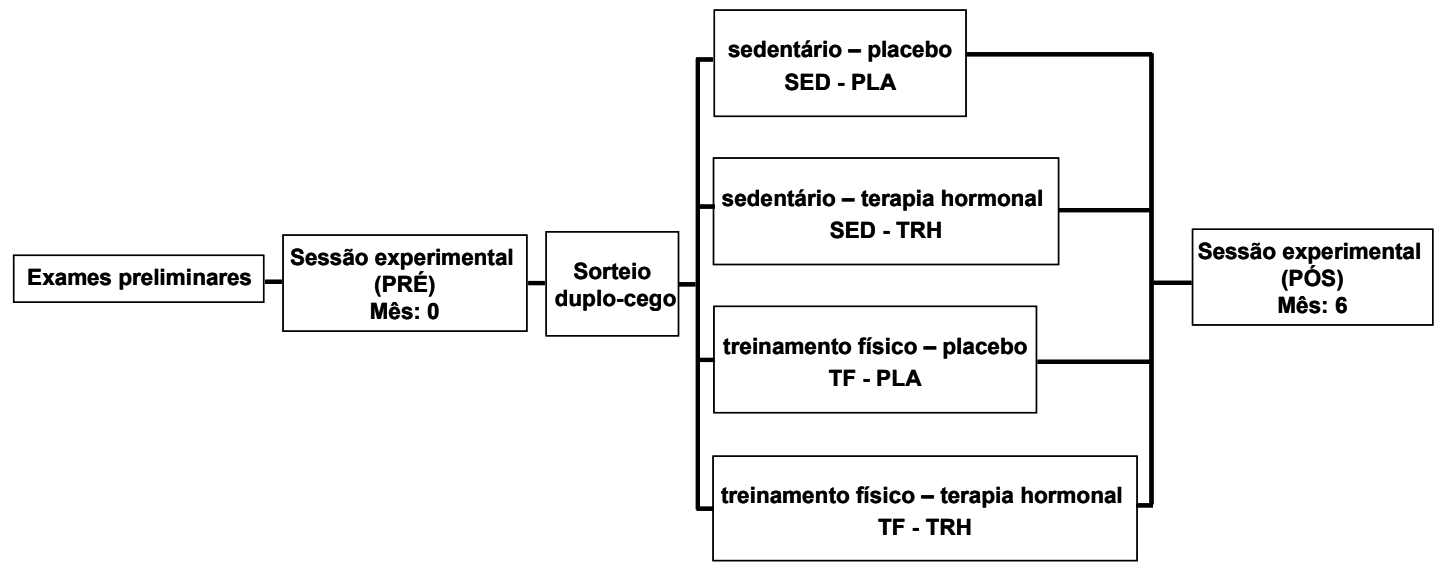

\subsubsection{Sessões Experimentais}

O fluxograma das sessões experimentais está ilustrado na Figura 2.

Nas duas sessões experimentais as voluntárias deveriam chegar ao laboratório após duas horas da ultima refeição. Nenhum tipo de exercício físico poderia ser realizado nas 48 horas precedentes ao experimento.

Após esvaziar a bexiga a voluntária era orientada a deitar na maca na posição supinada. Era então realizado o teste de contração voluntária máxima (íten 3.5.3) e colocados os equipamentos de medida de pressão arterial sistólica (PAS), diastólica (PAD) e média (PAM), freqüência cardíaca (FC) e fluxo sanguíneo do antebraço (FSA) e os procedimentos iniciais da microneurografia eram iniciados. Uma vez localizado o sinal nervoso, eram realizados 10 minutos de repouso. Neste período, denominado de basal, a atividade simpática periférica era registrada continuamente, a pressão arterial, freqüência cardíaca e o fluxo sanguíneo do antebraço eram medidos de minuto em minuto nos últimos 5 minutos. Em seguida, eram realizados os exercícios estático e dinâmico com "handgrip" em ordem aleatória conforme 
descrito no item 3.5.3, com período de pelo menos 5 minutos de intervalo entre eles para que as variáveis hemodinâmicas e neurais retornassem aos valores basais.

Figura 2 - Fluxograma das sessões experimentais

Chegada da paciente ao laboratório;

Esvaziamento da bexiga;

Realização do teste de contração voluntária máxima;

Colocação dos equipamentos

\section{Microneurografia}
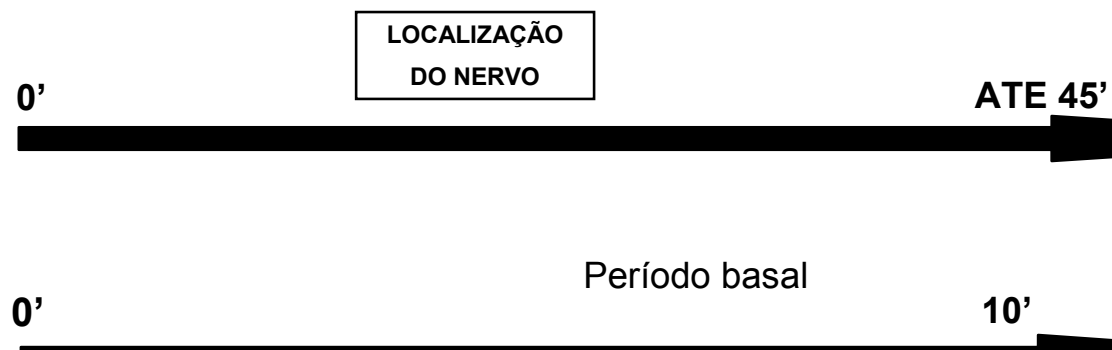

Medidas: PAS, PAD, PAM, FC, ANSP, FSA

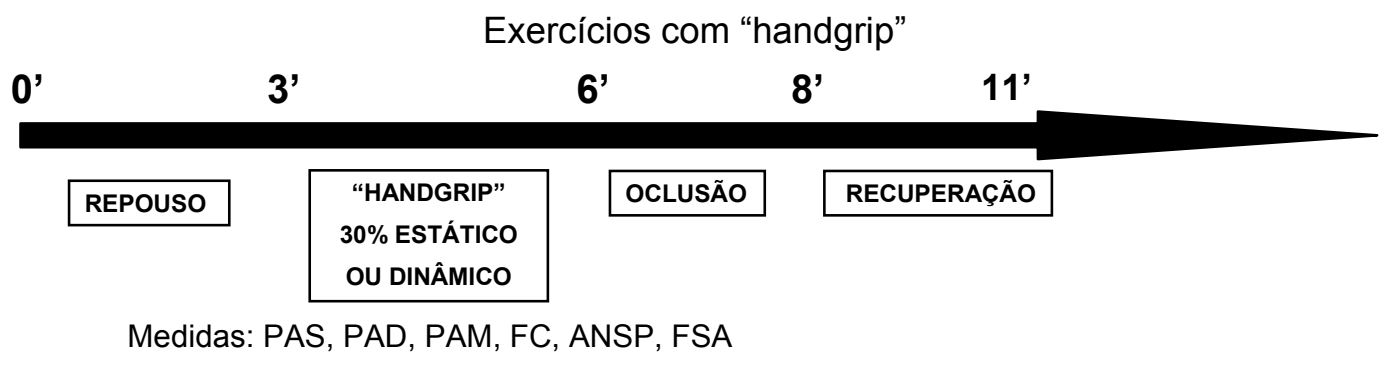




\subsection{Exames Preliminares}

\subsubsection{Avaliação de menopausa}

A presença da menopausa foi avaliada através de abordagem direta (dosagens hormonais). Foram incluídas no estudo as voluntárias que apresentaram elevações de FSH $(>35 \mu \mathrm{m} / \mathrm{ml})$ e LH $(>25 \mu \mathrm{m} / \mathrm{ml})$ e diminuição de Estradiol $(<40 \mathrm{pg} / \mathrm{ml})$. Essa avaliação ficou a cargo de médicas ginecologistas do Ambulatório de Ginecologia e Obstetrícia do Hospital das Clínicas da Faculdade de Medicina da Universidade de São Paulo.

\subsubsection{Avaliação dos sintomas da menopausa}

Os sintomas da menopausa foram avaliados pelo índice de Kupperman apresentado no Anexo C. Esse questionário foi preenchido na forma de entrevista.

\subsubsection{Avaliação de obesidade}

O peso e a estatura foram medidos numa balança (Welmy) e o índice de massa corporal (IMC) foi calculado pelo quociente entre o peso $(\mathrm{kg})$ e 0

quadrado da estatura $\left(\mathrm{m}^{2}\right)$. Não foram incluídas no estudo, as mulheres com IMC superior a $30 \mathrm{~kg} / \mathrm{m}^{2}$.

\subsubsection{Avaliação cardiovascular}

A condição cardiovascular foi avaliada por exame clínico e pela execução do eletrocardiograma de repouso e exercício. Foram excluídas as mulheres que apresentaram alterações indicativas de problemas cardíacos. 


\subsubsection{Avaliação de diabetes}

Foi realizada uma coleta de sangue após jejum de 12 horas. Foram excluídas do estudo as mulheres diabéticas, segundo o critério do "Expert Committee of the Diagnosis and Classification of Diabetes Mellitus" ${ }^{\prime \prime}$, que consiste de glicemia de jejum superior a $125 \mathrm{mg} / \mathrm{dL}$.

\subsubsection{Avaliação de hipercolesterolemia}

Juntamente com a coleta de sangue para glicemia foi coletada uma amostra de sangue, para a dosagem de triglicérides, colesterol total e frações. Foram excluídas do estudo, as mulheres que apresentassem valores de colesterol total acima de $239 \mathrm{mg} / \mathrm{dL}$, seguindo-se os critérios do "National Cholesterol Education Program"70, que na data do início do estudo eram os vigentes.

\subsubsection{Avaliação de hipertensão}

As voluntárias tiveram suas pressões arteriais medidas por três vezes, após cinco minutos de repouso na posição sentada, em duas visitas ao laboratório, como sugere o "Joint of the National Committee on Detection, Evaluation and Treatment of High Blood Pressure"71. A pressão arterial foi verificada pelo método auscultatório, utilizando-se esfigmomanômetro de coluna de mercúrio e considerando-se as fases I e V dos sons de Korotkoff para a identificação das pressões arteriais sistólica e diastólica, respectivamente. Apenas foram incluídas no estudo, as voluntárias normotensas na admissão, ou seja, aquelas que apresentaram valores de 
pressão arterial médio das seis medidas realizadas inferiores a $140 \mathrm{~mm} \mathrm{Hg}$ para a pressão arterial sistólica e $90 \mathrm{~mm} \mathrm{Hg}$ para a pressão arterial diastólica.

\subsubsection{Avaliação da capacidade aeróbia máxima}

Foi avaliada por um teste ergoespirométrico, realizado em cicloergômetro (Corival Cycle), empregando-se o protocolo escalonado com incremento de $30 \mathrm{~W}$ a cada 3 minutos até a exaustão física. Essa avaliação foi precedida pela execução de um eletrocardiograma de repouso (Cardio Perfect) com registro das 12 derivações padrão (D1, D2, D3, aVL, aVR, aVF, V1, V2, V3, V4, V5 e V6). Durante o esforço físico, a freqüência cardíaca e o eletrocardiograma foram continuamente monitorados e registrados ao final de cada estágio. As pressões arteriais sistólica e diastólica foram medidas pelo método auscultatório, através de um esfigmomanômetro de coluna de mercúrio imediatamente antes do registro da freqüência cardíaca. Os gases expirados foram coletados e analisados a cada ciclo respiratório pelo analisador de gases computadorizado da Medical Graphics Corporation (CPX/D) durante todo o protocolo de teste. A capacidade aeróbia máxima foi avaliada pelo consumo de oxigênio no final do exercício $\left(\mathrm{VO}_{2}\right.$ pico), calculados em média de 30 segundos. A determinação dos limiares ventilatórios seguiram às recomendações de Bhambhani e Singh ${ }^{72}$, ou seja, o limiar anaeróbio foi determinado pela perda de linearilidade de incremento da razão de troca respiratória $\left(\mathrm{RER}=\mathrm{VCO}_{2} / \mathrm{VO}_{2}\right)$, pela primeira perda da linearilidade da ventilação (VE), pela menor pressão expirada final de 
oxigênio $\left(\mathrm{PetO}_{2}\right)$ e pelo menor equivalente ventilatório de oxigênio $\left(\mathrm{VE} / \mathrm{VO}_{2}\right)$, enquanto que o ponto de compensação respiratória foi determinado pela segunda perda da linearilidade de incremento da ventilação (VE) e da freqüência respiratória $(F R)$, pelo menor equivalente respiratório de dióxido de carbono $\left(\mathrm{VE} / \mathrm{VCO}_{2}\right)$ e pela maior pressão expirada final de dióxido de carbono $\left(\mathrm{PetCO}_{2}\right)$.

\subsection{Procedimentos experimentais}

\subsubsection{Terapia estrogênica (TRH) ou placebo (PLA)}

Para as voluntárias alocadas no grupo $\mathrm{TRH}$, foi administrado valerato estradiol (comercializado como Primogyna®) $1 \mathrm{mg} / \mathrm{dia}$ enquanto que, para aquelas do grupo PLA, foi administrado placebo. Os dois grupos eram orientados a ingerir uma drágea ao dia por via oral, de forma contínua e durante 6 meses. Essa administração foi realizada de forma aleatória e duplo-cega.

A dose da medicação utilizada no estudo foi aquela já testada em ensaios clínicos de apoio, ou seja, estavam em conformidade com os documentos aprovados pelo Ministério da Saúde para comercialização no mercado brasileiro. Da mesma forma, as características farmacológicas do placebo estavam em conformidade com as exigências das autoridades sanitárias e sua utilização se justifica por se tratar de metodologia científica apurada e precisa.

Para cada mulher recrutada, foi atribuído um número de alocação de acordo com uma lista de aleatorização previamente elaborada 
eletronicamente. A partir da visita de alocação e a cada visita subseqüente, as voluntárias recebiam, gratuitamente, 02 frascos contendo cada um, 28 drágeas da medicação do estudo (valerato de estradiol $1 \mathrm{mg}$ ) ou placebo. Ao receber a droga, a voluntária estava assumindo o compromisso de utilizá-las apenas para fins do estudo. Todos os frascos vazios ou contendo drágeas não utilizadas deveriam ser devolvidos ao investigador na visita subseqüente, condição para que 02 novos frascos fossem fornecidos à voluntária.

A medicação do estudo ou o placebo eram suspensos e a mulher seria excluída do estudo nas seguintes situações:

- Ocorrência de qualquer efeito adverso sério;

- Perda de seguimento do estudo;

As condutas clínicas relacionadas à terapia hormonal estrogênica ficaram a cargo da Profa. Dra. Angela Maggio da Fonseca do serviço de Ginecologia do Hospital das Clínicas da Faculdade de Medicina da Universidade de São Paulo (HC/FMUSP).

\subsubsection{Treinamento físico (TF) ou sedentarismo (SED)}

Durante o estudo, as voluntárias alocadas no grupo TF foram submetidas a treinamento físico com freqüência de três sessões semanais. Cada sessão era composta de cinco minutos de aquecimento em cicloergômetro, 20 a 50 minutos de exercício aeróbio em cicloergômetro, cinco minutos de recuperação e 10 minutos de alongamento/relaxamento. A intensidade inicial do treinamento era fixada pela freqüência cardíaca correspondente ao Limiar Anaeróbio (LA) no teste máximo e progredia gradualmente até atingir a 
freqüência cardíaca correspondente ao Ponto de Compensação Respiratória (PCR). Para tanto, a progressão da potência e duração do treinamento eram sempre ajustados pela diminuição da freqüência cardíaca durante as sessões de treinamento. As mulheres alocadas no grupo SED eram instruídas a não modificar a sua prática de atividade física no período do estudo.

\subsubsection{Exercícios estático e dinâmico com "handgrip"}

Os exercícios estático ou dinâmico com "handgrip" foram realizados para testar a alça eferente simpática do reflexo pressorreceptor ${ }^{29}$. O exercício estático caracteriza-se por contração muscular mantida, isto é, sem relaxamento, por tempo pré-estabelecido, enquanto que o exercício dinâmico caracteriza-se por uma seqüência de contrações e relaxamento da musculatura. Ambos podem ser realizados num percentual da força de contração máxima do indivíduo e são capazes de promover grande aumento da pressão arterial causada tanto por uma diminuição parassimpática como por um aumento da atividade eferente simpática ${ }^{73}$.

A medida da contração voluntária máxima foi realizada em três tentativas consecutivas no início da sessão ("handgrip", Stoelting).

Exercício estático: a voluntária realizava a preensão estática da mão não dominante por 3 minutos $30 \%$ da contração voluntária máxima.

Exercício dinâmico: a voluntária realizava a preensão dinâmica na freqüência de 40 contrações/min por três minutos em 30\% da contração voluntária máxima, com auxílio de um metrônomo. 
Tanto o exercício estático (EE) como o dinâmico (ED) eram precedidos de 3 minutos de registro de repouso (REP) e seguido de 2 minutos de oclusão (OCLU) da circulação do braço não dominante realizada com manguito pneumático a uma pressão de $200 \mathrm{~mm} \mathrm{Hg}$ e 3 minutos de recuperação (REC). O REP, EE ou ED, OCLU e REC foram chamados de estágios do exercício estático ou dinâmico.

\subsection{Medidas durante sessões experimentais}

\subsubsection{Pressão arterial e freqüência cardíaca}

Pressão arterial sistólica, diastólica e média e freqüência cardíaca foram medidas por um aparelho oscilométrico automático (Dixtal, 2710) validado ${ }^{74}$, regularmente calibrado e comparado com coluna de mercúrio. Esse equipamento foi colocado na perna esquerda das voluntárias.

\subsubsection{Fluxo sangüíneo do antebraço}

O fluxo sanguíneo do antebraço (FSA) foi medido pela técnica da pletismografia de oclusão venosa $a \mathrm{ar}^{75}$. Resumidamente consiste em: o braço direito da voluntária é mantido acima do nível do coração e dois manguitos são posicionados, um no punho e outro no braço. Um manguito de látex preenchido com ar até uma pressão de $4 \mathrm{~cm}$ de água é colocado no antebraço. Para se determinar o fluxo sanguíneo, o manguito do punho é inflado até $200 \mathrm{~mm} \mathrm{Hg}$, interrompendo a circulação sangüínea para a mão, e o manguito do braço é inflado (até uma pressão sub-diastólica de 40 a 60 $\mathrm{mmHg}$ ) e desinflado em intervalos de 7 a 8 segundos. Esse procedimento 
provoca a oclusão do retorno venoso sem interromper a entrada arterial de sangue no antebraço, ocasionando dilatação nessa região, que provoca aumento na pressão interna no manguito de látex, que é registrado no polígrafo (GOULD RS3000). O fluxo sangüíneo do antebraço é calculado através da velocidade de incremento da pressão no manguito de látex.

\subsubsection{Atividade nervosa simpática periférica}

A atividade nervosa simpática periférica (ANSP) foi medida pela técnica de microneurografia ${ }^{76,}{ }^{77}$. Essa técnica consiste na colocação de microeletrodos de tungstênio $(30-40 \mathrm{~mm}$ de comprimento, $0,2 \mathrm{~mm}$ de diâmetro com ponta de $1-5 \mu \mathrm{m})$, flexíveis, que permitem a inserção percutânea sem anestesia local em nervos periféricos para o registro de potenciais de ação ocasionando desconforto mínimo.

O procedimento envolve três passos básicos:

1- Estimulação elétrica percutânea do nervo para mapeamento de seu trajeto (40 a $120 \mathrm{~V}, 0,2 \mathrm{~ms}$ e $1 \mathrm{~Hz})$, promove contrações musculares involuntárias e/ou parestesia na região inervada, que orientam na demarcação do trajeto;

2- Estimulação interna (4-5V, 0,2ms e 1Hz). Inserção de dois eletrodos, um ativo (percutâneamente intraneural) e um de referência (subcutaneamente, $1-2 \mathrm{~cm}$ do eletrodo ativo);

3- Ajustes finos da posição do eletrodo ativo a fim de obter um local de registro satisfatório ${ }^{78}$. 
Esse procedimento deveria ser completado em, no máximo, 45 minutos. Caso o nervo não fosse localizado, a sessão era então cancelada e remarcada para 15 dias. Caso o nervo fosse localizado, seu sinal era amplificado, filtrado e integrado sendo registrado em papel através de um polígrafo GOULD RS 3000. A atividade nervosa simpática foi medida, contando-se o número de impulsos nervosos por minuto.

\subsection{Análise dos dados}

No período basal, os valores de atividade nervosa simpática periférica, pressões arteriais, freqüência cardíaca, fluxo sangüíneo do antebraço e resistência vascular do antebraço foram calculados pela média das medidas

realizadas durante os últimos 5 minutos do período para garantir a estabilização das variáveis fisiológicas.

Durante os exercícios estático e dinâmico com "handgrip" foram realizadas as médias dos 3 minutos de repouso, dos 3 minutos de realização dos exercícios, dos 2 minutos de oclusão da circulação sanguínea do antebraço e dos 3 minutos de recuperação de cada um deles.

\subsection{Análise estatística}

No período basal, os valores da atividade nervosa simpática periférica, pressões arteriais (sistólica, média e diastólica), freqüência cardíaca e fluxo sanguíneo do antebraço, nas sessões PRÉ e PÓS, foram comparados pela análise de variância (ANOVA) de três fatores, tendo como fatores principais independentes: terapia (placebo - PLA ou terapia hormonal estrogênica - 
TRH) e condição (sedentário - SED ou treinado - TF), e dependente: sessões (início - PRÉ e seis meses de acompanhamento - PÓS).

Para os exercícios estático e dinâmico com "handgrip", foi realizada análise de variância ANOVA de quatro fatores, tendo como fatores principais independentes: terapia (PLA ou TRH) e o treinamento físico (SED ou TF), e como fatores dependentes: a sessão (PRÉ e PÓS) e os estágios (repouso, exercício, oclusão e recuperação).

Quando houve significância, a mesma foi localizada empregando-se o teste de "post- hoc" de Newman-Keuls e foi considerado como significativo o índice de $\mathrm{P}<0,05$. Em todas as circunstâncias, os dados foram apresentados como média e desvio padrão (dp) da média. 


\section{RESULTADOS}

\subsection{Gerais}

Os dados desta tese foram coletados no período de setembro de 2002 a dezembro de 2008.

Um número de 430 pacientes realizou entrevista para participar do estudo. Destas, 61 preenchiam os critérios de inclusão do estudo. Um total de 60 pacientes realizaram a sessão PRÉ sendo que cada grupo continha 20 pacientes. Cinqüenta e uma sessões PRÉ foram bem sucedidas. Destas, 12 foram de pacientes do grupo SED-PLA, 14 do grupo SED-TRH, 14 do grupo TF-PLA e 11 do grupo TF-TRH. No período entre as sessões PRÉ e PÓS, 3 voluntárias foram excluídas: 1 paciente do grupo TF-PLA devido a crises freqüentes de asma que a impossibilitavam de realizar o treinamento físico, uma do grupo TF-PLA e outra do grupo TF-TRH, desistiram do estudo.

Dessa forma, 48 pacientes realizaram a sessão PÓS e, destas 45 sessões foram bem sucedidas compondo a amostra do estudo: 11 pacientes do grupo SED-PLA, 14 do grupo SED-TRH, 12 do grupo TF-PLA e 8 do grupo TF-TRH.

Foram consideradas sessões bem sucedidas, quando o nervo era localizado e apresentava um registro legível, que pudesse ser analisado, futuramente. Foram consideradas sessões mal sucedidas, quando o nervo 
não era localizado, ou que não se obtinha um bom registro, após duas tentativas consecutivas, com a mesma paciente, em intervalo de 15 dias. Também foram consideradas sessões mal sucedidas quando ocorria algum problema técnico em qualquer equipamento, que impedisse a continuidade da sessão experimental.

O tempo entre a histerectomia e o ingresso no estudo foi de $8 \pm 6$ anos.

Os quatro grupos não apresentaram diferenças estatisticamente significante no peso, estatura, IMC, PAS, PAD e PAM quando comparado PRÉ e PÓS.

O índice de Kupperman na sessão PÓS foi significativamente mais baixo do que na sessão PRÉ nos dois grupos que utilizaram terapia hormonal $(P<0,01$ para SED-TRH e $P=0,03$ para TF-TRH).

$\mathrm{O} \mathrm{VO}_{2}$ máximo foi mais alto nos grupos que realizaram treinamento físico ( $P=0,04$ para TF-TRH e $P<0,01$ para TF-PLA) quando sessão PRÉ e PÓS foram comparadas. Esses dados são apresentados na Tabela 1. A terapia estrogênica não alterou as respostas de $\mathrm{VO}_{2}$ máximo das voluntárias $(P=0,2)$. 


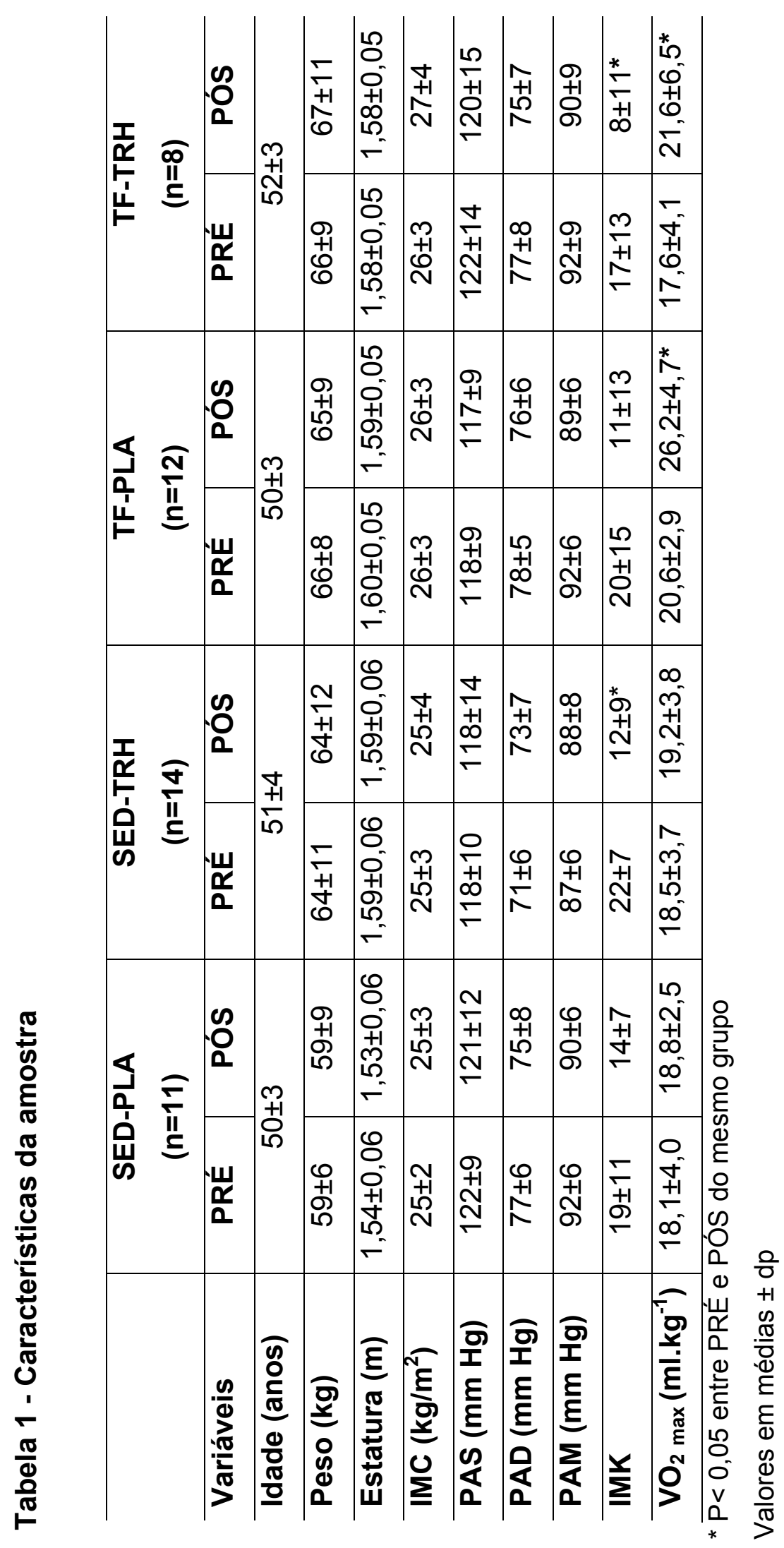


A Tabela 2 apresenta os resultados dos exames laboratoriais dos grupos nas sessões PRÉ e PÓS. O nível de estradiol foi significativamente mais alto na sessão PÓs dos grupos SED-TRH $(P<0,01)$ e TF-TRH $(P=0,04)$. Os níveis de LH no grupo SED-TRH $(P=0,04)$ foram significativamente mais baixos na sessão PÓS.

Tabela 2 - Médias dos valores dos exames laboratoriais dos grupos no início (PRÉ) e após 6 meses de estudo(PÓS)

\begin{tabular}{l|c|c|c|c|c|c|c|c}
\hline & & \multicolumn{2}{c|}{ SED-TRH } & \multicolumn{2}{c}{ TF-PLA } & \multicolumn{2}{c}{ TF-TRH } \\
\cline { 2 - 9 } & PRÉ & PÓS & PRÉ & PÓS & PRÉ & PÓS & PRÉ & PÓS \\
\hline Colesterol total (mg/dL) & $216 \pm 21$ & $211 \pm 34$ & $215 \pm 18$ & $212 \pm 27$ & $202 \pm 27$ & $196 \pm 25$ & $207 \pm 31$ & $199 \pm 23$ \\
\hline Glicemia (mg/dL) & $88 \pm 10$ & $91 \pm 12$ & $89 \pm 10$ & $86 \pm 9$ & $87 \pm 6$ & $90 \pm 8$ & $88 \pm 10$ & $86 \pm 7$ \\
\hline Estradiol (pg/ml) & $17 \pm 8$ & $17 \pm 10$ & $14 \pm 2$ & $47 \pm 28^{*}$ & $18 \pm 8$ & $17 \pm 4$ & $19 \pm 11$ & $48 \pm 31^{*}$ \\
\hline LH $(\mu \mathrm{m} / \mathrm{ml})$ & $36 \pm 16$ & $37 \pm 13$ & $42 \pm 17$ & $37 \pm 17^{*}$ & $36 \pm 16$ & $32 \pm 15$ & $37 \pm 11$ & $37 \pm 17$ \\
\hline FSH $(\mu \mathrm{m} / \mathrm{ml})$ & $85 \pm 29$ & $88 \pm 27$ & $84 \pm 35$ & $68 \pm 34$ & $77 \pm 30$ & $74 \pm 43$ & $81 \pm 25$ & $64 \pm 19$ \\
\hline
\end{tabular}

* $\mathrm{P}<0,05$ entre PRÉ e PÓS do mesmo grupo

Valores em média $\pm d p$

\subsection{Período basal}

As médias de todas as variáveis analisadas no estudo, separadas por grupo encontram-se na Tabela 3.

A média de ANSP na sessão PÓS dos dois grupos que treinaram (TRH e PLA) foi significativamente menor do que na sessão PRÉ dos mesmos grupos $(P=0,01)$, conforme demonstra Figura 3 . 
Figura 3 - Representação gráfica das respostas da ANSP dos grupos TF e SED nas sessões PRÉ e PÓS no período basal

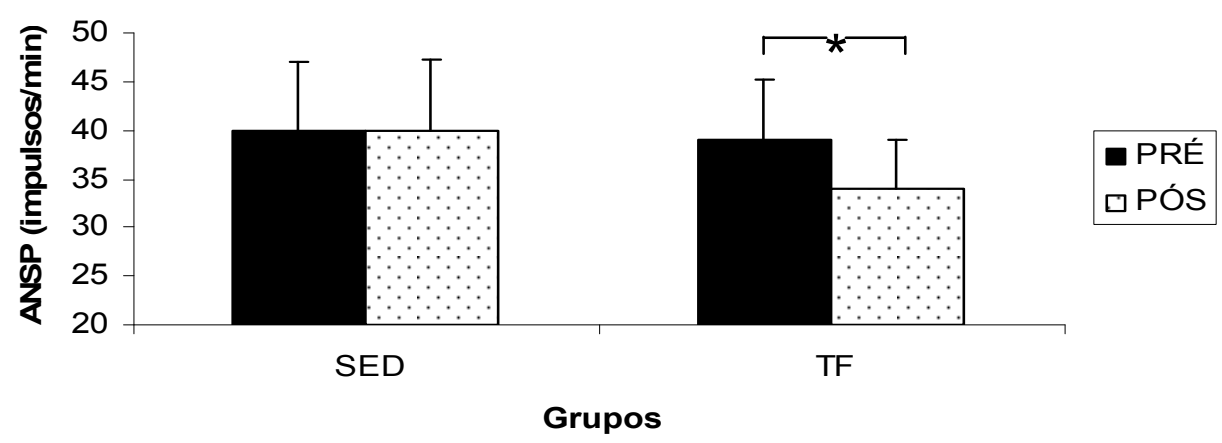

${ }^{*} \mathrm{P}<0,05$

Quando os grupos foram analisados isoladamente, foi observado que a ANSP e a PAS do grupo TF-PLA-PÓS foram significativamente menores que o mesmo grupo, na sessão $P R E ́$ ( $P=0,02$ e $P=0,03$ respectivamente).

A FC do grupo TF-TRH-PÓS foi menor do que o mesmo grupo na sessão PRÉ $(P=0,01)$.

A média de FC da sessão PÓS de todos os grupos $(65 \pm 10 \mathrm{bpm})$ foi significativamente mais baixa do que a sessão PRÉ (68 $\pm 11 \mathrm{bpm})$, independentemente do treinamento físico e da terapia hormonal $(P=0,04)$.

A média de PAD de todos os grupos juntos, na sessão PÓS foi significativamente menor do que sessão PRÉ $74 \pm 8$ vs $76 \pm 9 \mathrm{~mm} \mathrm{Hg}$ $(P=0,02)$, independentemente do treinamento e da terapia hormonal.

O FSA foi significativamente maior na sessão PÓS dos dois grupos que realizaram o treinamento físico $[2,65 \pm 1,34 \mathrm{ml}(\min .100 \mathrm{ml})]$, comparado ao 
PRÉ dos mesmos grupos [1,92 $0,96 \mathrm{ml}(\min .100 \mathrm{ml})]$, independentemente do tipo de medicamento utilizado (Figura 4) .

Uma análise dos grupos separadamente observou que a ANSP do grupo TF-PLA-PÓS foi significativamente menor que o mesmo grupo, na sessão PRÉ $(P=0,01)$.

A FC do grupo TF-TRH-PÓS foi menor do que o mesmo grupo na sessão PRÉ $(P=0,01)$. Estes dados encontram-se na Tabela 3.

Figura 4 - Representação gráfica das respostas do FSA dos grupos TF e SED nas sessões PRÉ e PÓS no período basal

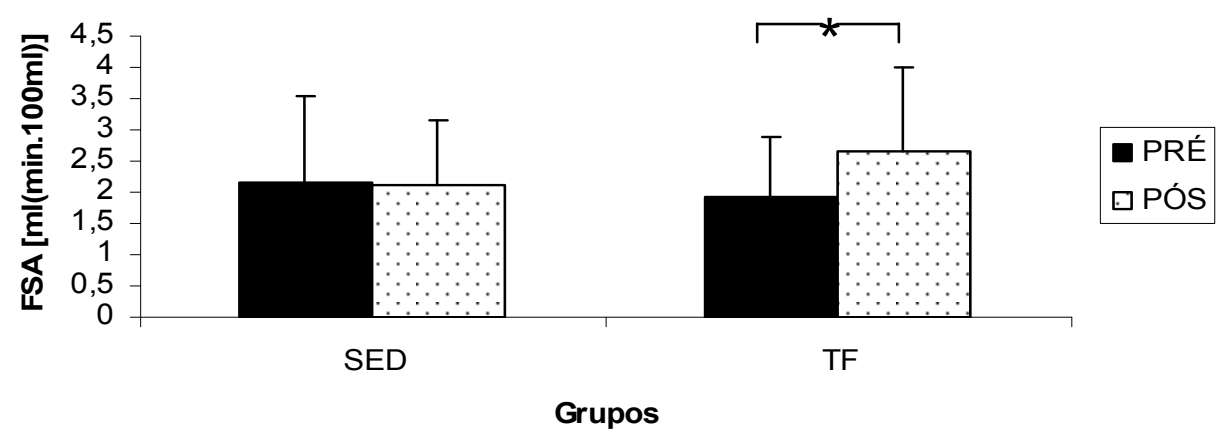

${ }^{*} \mathrm{P}<0,05$ 


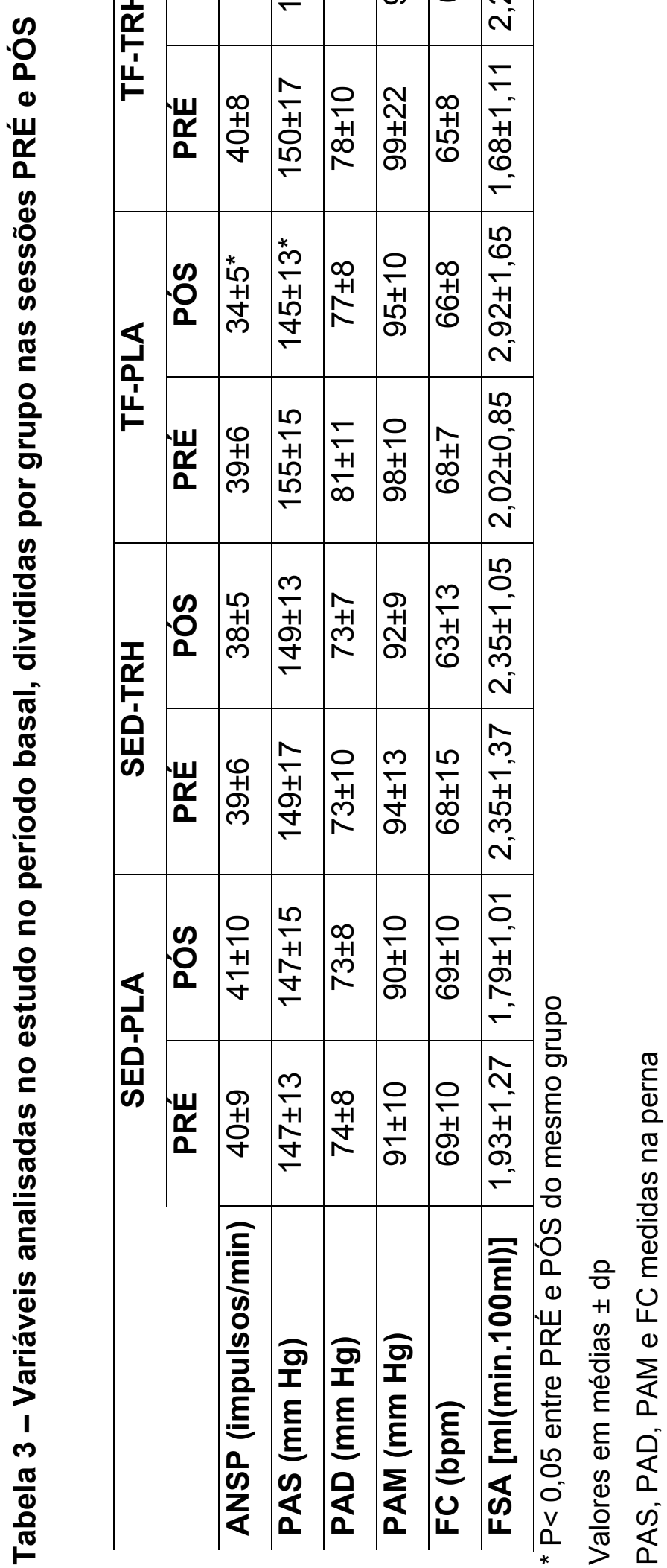




\subsection{Exercícios estático e dinâmico com "handgrip"}

\subsubsection{Atividade nervosa simpática periférica}

Isoladamente, nem o treinamento físico $(P=0,2)$, nem o medicamento $(P=0,6)$ influenciaram a ANSP durante os estágios do exercício estático. Não houve diferença entre sessões PRÉ e PÓS $(P=0,2)$ durante os mesmos estágios.

Todos os grupos evidenciaram aumento na ANSP na realização do EE $(P<0,01)$. A ANSP se manteve elevada na OCLU $(P=0,7$ vs $E E)$ e na REC diminuiu seu valor ( $\mathrm{P}<0,01$ vs $\mathrm{EE}$ e $\mathrm{P}=0,5$ vs $\mathrm{REP}$ ). $\mathrm{O}$ comportamento dessas variáveis pode ser observado na Figura 3. A Tabela 4 demonstra as médias de ANSP nos estágios do exercício estático dos 4 grupos.

Tabela 4 - Respostas de ANSP (impulsos/min) dos grupos durante os estágios do exercício estático com "handgrip" nas sessões PRÉ e PÓS

\begin{tabular}{c|ccccc}
\hline Grupo & Sessões & Rep & EE30\% & Oclu & Rec \\
\hline SED-PLA & PRÉ & $42 \pm 9$ & $48 \pm 8$ & $48 \pm 10$ & $44 \pm 10$ \\
& PÓS & $41 \pm 10$ & $47 \pm 8$ & $47 \pm 8$ & $41 \pm 7$ \\
\cline { 2 - 6 } SED-TRH & PRÉ & $42 \pm 10$ & $46 \pm 10$ & $45 \pm 8$ & $43 \pm 10$ \\
& PÓS & $39 \pm 6$ & $44 \pm 8$ & $45 \pm 7$ & $39 \pm 6$ \\
\cline { 2 - 6 } TF-PLA & PRÉ & $38 \pm 9$ & $46 \pm 11$ & $47 \pm 8$ & $40 \pm 13$ \\
& PÓS & $36 \pm 6$ & $44 \pm 8$ & $43 \pm 4$ & $37 \pm 6$ \\
\cline { 2 - 6 } TF-TRH & PRÉ & $40 \pm 7$ & $42 \pm 8$ & $44 \pm 8$ & $39 \pm 7$ \\
& PÓS & $38 \pm 9$ & $41 \pm 9$ & $40 \pm 10$ & $38 \pm 8$ \\
\hline
\end{tabular}

Valores em média $\pm d p$ 
Com relação aos estágios dos exercícios dinâmicos, de maneira isolada, nem o TF $(P=0,9)$ nem a TRH $(P=0,6)$ influenciaram a ANSP. Não houve diferença na ANSP entre as sessões PRÉ e PÓS $(P=0,6)$.

Foi observada uma elevação da ANSP na realização do ED $(P<0,01$ vs REP). Na OCLU, a ANSP se manteve elevada ( $P=0,8$ comparado ao ED) e na REC a ANSP foi diminuída comparado à OCLU ( $P<0,01$ vs OCLU), porém esse valor permaneceu maior do que o repouso inicial $(P=0,02$ vs REP). O comportamento dessa variável pode ser observado na Figura 5. A Tabela 5 demonstra as médias de ANSP dos quatro grupos nas duas sessões.

Tabela 5 - Respostas de ANSP (impulsos/min) dos grupos durante os estágios do exercício dinâmico com "handgrip" nas sessões PRÉ e PÓS

\begin{tabular}{c|ccccc}
\hline Grupo & Sessões & Rep & ED30\% & Oclu & Rec \\
\hline SED-PLA & PRÉ & $41 \pm 12$ & $45 \pm 11$ & $44 \pm 10$ & $46 \pm 14$ \\
& PÓs & $37 \pm 7$ & $43 \pm 7$ & $44 \pm 8$ & $39 \pm 11$ \\
\hline SED-TRH & PRÉ & $37 \pm 11$ & $40 \pm 13$ & $40 \pm 15$ & $36 \pm 13$ \\
& PÓs & $35 \pm 7$ & $39 \pm 9$ & $38 \pm 8$ & $37 \pm 8$ \\
\hline TF-PLA & PRÉ & $36 \pm 7$ & $40 \pm 5$ & $71 \pm 8$ & $36 \pm 9$ \\
& PÓs & $38 \pm 8$ & $43 \pm 8$ & $43 \pm 8$ & $42 \pm 4$ \\
\hline TF-TRH & PRÉ & $39 \pm 9$ & $43 \pm 10$ & $43 \pm 9+$ & $40 \pm 8$ \\
& PÓs & $39 \pm 4$ & $40 \pm 10$ & $39 \pm 8$ & $39 \pm 6$ \\
\hline
\end{tabular}

Valores em média $\pm \mathrm{dp}$

Quando as médias dos estágios do exercício estático foram comparadas aos exercícios dinâmicos, observou-se que a ANSP foi significativamente mais alta no EE e OCLU após EE, comparadas a ED $(P=0,03)$ e OCLU após ED ( $P=0,01)$, conforme demonstra Figura 5. 
Figura 5 - Representação gráfica do comportamento da ANSP durante estágios do exercício estático e dinâmico com "handgrip" de todos os grupos, nas duas sessões

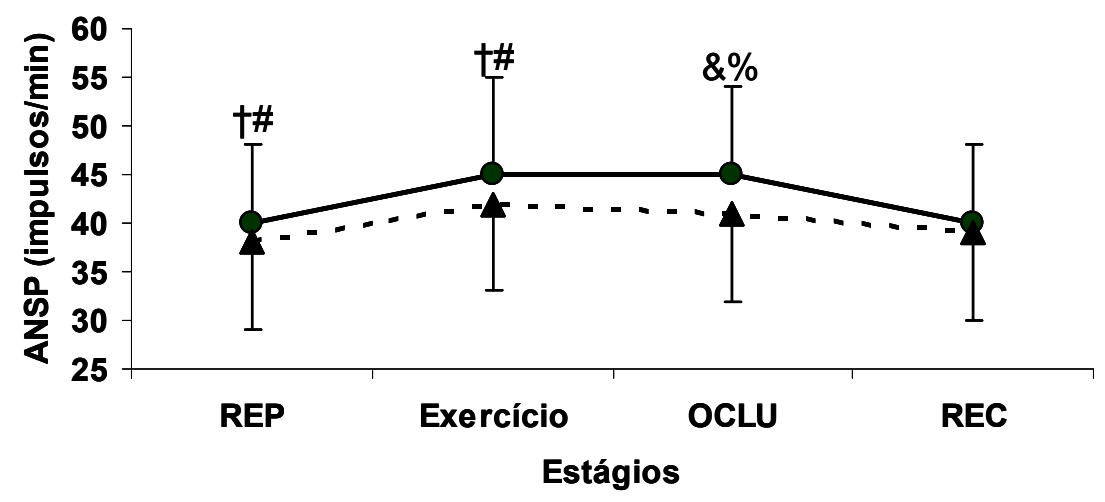

- Exercício estático ---- Exercício dinâmico
† $\mathrm{P}<0,05$ entre estágios dos exercícios estático e dinâmico
\# $\mathrm{P}<0,05$ comparado ao estágio REP para exercícios estático e dinâmico
\& $\mathrm{P}<0,05$ comparado ao estágio exercício para exercícios estático e dinâmico
$\% \mathrm{P}<0,05$ comparado ao estágio REP apenas para exercício dinâmico

\subsubsection{Pressão arterial sistólica}

O treinamento físico $(P=0,4)$ e o uso do medicamento $(P=0,8)$ não influenciaram a PAS na realização dos estágios do EE. Não houve diferença entre as sessões PRÉ e PÓS $(P=0,3)$. Todos os grupos sofreram aumento de PAS durante a realização do $E E(P<0,01$ vs repouso). A PAS se manteve elevada no período da OCLU ( $P=0,5$ vs EE) e na REC diminuiu seu valor, retornando a valor semelhante ao REP ( $P=0,9$ vs REP). $O$ comportamento dessas variáveis está ilustrado na Figura 6 e os valores de cda grupo nas sessões PRÉ e PÓS encontram-se na Tabela 6. 
Tabela 6 - Respostas de PAS $(\mathrm{mm} \mathrm{Hg})$ dos grupos durante os estágios do exercício estático com "handgrip" nas sessões PRÉ e PÓS

\begin{tabular}{c|ccccc}
\hline Grupo & Sessões & Rep & EE30\% & Oclu & Rec \\
\hline SED-PLA & PRÉ & $148 \pm 17$ & $158 \pm 16$ & $153 \pm 17$ & $146 \pm 15$ \\
& PÓS & $148 \pm 13$ & $164 \pm 11$ & $160 \pm 15$ & $142 \pm 19$ \\
\hline SED-TRH & PRÉ & $150 \pm 17$ & $163 \pm 19$ & $161 \pm 20$ & $153 \pm 19$ \\
& PÓS & $150 \pm 15$ & $161 \pm 11$ & $156 \pm 13$ & $147 \pm 17$ \\
\hline TF-PLA & PRÉ & $155 \pm 22$ & $169 \pm 15$ & $172 \pm 19$ & $157 \pm 21$ \\
& PÓS & $145 \pm 14$ & $159 \pm 14$ & $164 \pm 20$ & $150 \pm 11$ \\
\hline TF-TRH & PRÉ & $153 \pm 25$ & $161 \pm 18$ & $165 \pm 21$ & $155 \pm 26$ \\
& PÓS & $154 \pm 24$ & $167 \pm 26$ & $164 \pm 28$ & $152 \pm 27$ \\
\hline
\end{tabular}

Valores em média $\pm d p$

De maneira isolada, nem o TF $(P=0,3)$ e nem a TRH $(P=0,7)$ influenciaram a PAS durante os estágios do ED. Não houve diferença entre as sessões PRÉ e PÓS $(P=1,0)$

Os grupos aumentaram PAS durante a realização do ED $(P<0,01$ vs repouso). A PAS se manteve elevada na OCLU ( $P=0,2$ vs ED) e, na REC retornou a valor semelhante ao inicial ( $P<0,01$ vs OCLU e $P=0,6$ vs REP), conforme demonstra Figura 6. A Tabela 7 apresenta as médias de cada grupo, em todos os estágios, sãs sessões PRÉ e PÓS. 
Tabela 7 - Respostas de PAS $(\mathrm{mm} \mathrm{Hg})$ dos grupos durante os estágios do exercício dinâmico com "handgrip" nas sessões PRÉ e PÓS.

\begin{tabular}{c|ccccc}
\hline Grupo & Sessões & Rep & ED30\% & Oclu & Rec \\
\hline SED-PLA & PRÉ & $147 \pm 13$ & $157 \pm 10$ & $153 \pm 15$ & $148 \pm 15$ \\
& PÓS & $150 \pm 11$ & $157 \pm 13$ & $154 \pm 17$ & $151 \pm 16$ \\
\hline SED-TRH & PRÉ & $149 \pm 18$ & $158 \pm 19$ & $158 \pm 19$ & $154 \pm 18$ \\
& PÓS & $146 \pm 14$ & $160 \pm 14$ & $155 \pm 14$ & $145 \pm 17$ \\
\hline TF-PLA & PRÉ & $150 \pm 20$ & $168 \pm 15$ & $164 \pm 21$ & $154 \pm 21$ \\
& PÓS & $150 \pm 16$ & $158 \pm 14$ & $159 \pm 16$ & $148 \pm 15$ \\
\hline TF-TRH & PRÉ & $156 \pm 26$ & $167 \pm 20$ & $166 \pm 21$ & $155 \pm 26$ \\
& PÓS & $161 \pm 12$ & $173 \pm 11$ & $173 \pm 11$ & $164 \pm 14$ \\
\hline
\end{tabular}

Valores em média $\pm d p$

Não houve diferença estatisticamente significante na PAS em nenhum dos estágios quando o EE foi comparado ao ED, conforme demonstra figura abaixo.

Figura 6 - Representação gráfica do comportamento da PAS durante estágios do exercício estático e dinâmico com "handgrip" de todos os grupos, nas duas sessões.

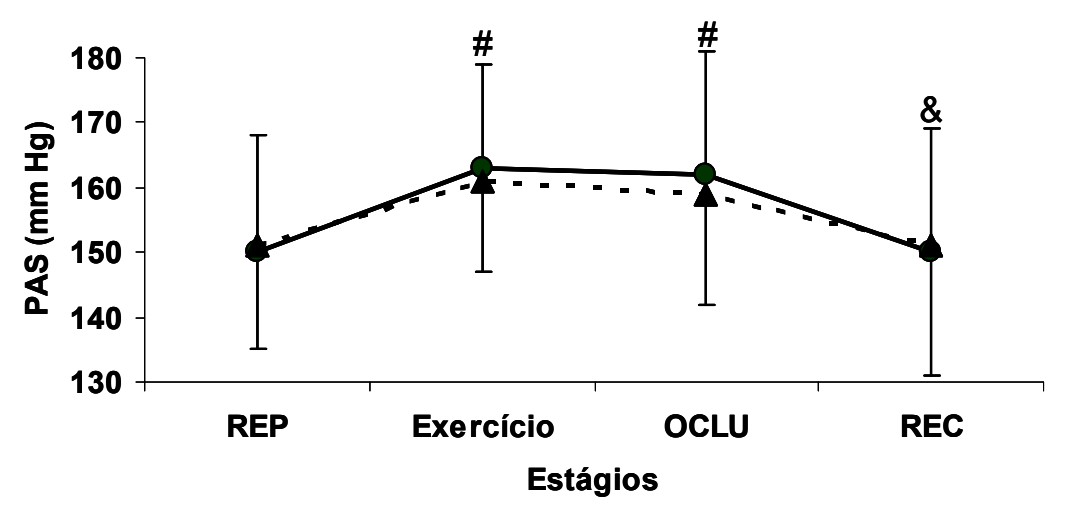

Exercício estático Exercício dinâmico

\# P $<0,05$ comparado ao estágio REP para exercícios estático e dinâmico $\& \mathrm{P}<0,05$ comparado ao estágio exercício para exercícios estático e dinâmico 


\subsubsection{Pressão arterial diastólica}

De maneira isolada, o TF $(P=0,9)$ e a TRH $(P=0,3)$ não influenciaram a PAD dos grupos durante os estágios do EE.

O comportamento da PAD nos estágios foi semelhante para todos os grupos: houve um aumento da PAD durante a realização do $\mathrm{EE}(\mathrm{P}<0,01$ vs repouso), na OCLU a PAD sofre uma atenuação ( $P<0,01$ vs $E E)$, mas se manteve elevada comparada ao REP $(P<0,01)$ e na REC a pressão retorna a valor semelhante ao repouso inicial $(P<0,01$ vs oclusão e $P=0,6$ vs repouso) (Figura 7). A Tabela 8 apresenta as médias de PAD dos grupos, em todos os estágios nas sessões PRÉ e PÓS.

Tabela 8 - Respostas de PAD (mm Hg) dos grupos durante os estágios do exercício estático com "handgrip" nas sessões PRÉ e PÓS

\begin{tabular}{c|ccccc}
\hline Grupo & Sessões & Rep & EE30\% & Oclu & Rec \\
\hline SED-PLA & PRÉ & $75 \pm 11$ & $85 \pm 10$ & $80 \pm 10$ & $74 \pm 10$ \\
& PÓS & $74 \pm 9$ & $87 \pm 14$ & $82 \pm 12$ & $75 \pm 10$ \\
\hline SED-TRH & PRÉ & $80 \pm 13$ & $94 \pm 17$ & $93 \pm 21$ & $83 \pm 15$ \\
& PÓS & $73 \pm 7$ & $86 \pm 8$ & $81 \pm 9$ & $74 \pm 6$ \\
\hline TF-PLA & PRÉ & $81 \pm 11$ & $99 \pm 14$ & $94 \pm 17$ & $82 \pm 12$ \\
& PÓS & $77 \pm 5$ & $90 \pm 11$ & $88 \pm 13$ & $78 \pm 6$ \\
\hline TF-TRH & PRÉ & $83 \pm 18$ & $92 \pm 18$ & $89 \pm 23$ & $81 \pm 19$ \\
& PÓS & $74 \pm 10$ & $91 \pm 17$ & $81 \pm 14$ & $73 \pm 11$ \\
\hline
\end{tabular}

Valores em média $\pm \mathrm{dp}$

$\mathrm{O} T F$ e a TRH isoladamente não influenciaram os valores de PAD ( $P=0,1$ e $P=0,8$ respectivamente) durante a realização dos estágios do ED. Todos os grupos sofreram aumento de PAD durante a realização do ED $(P<0,01$ vs REP). Na OCLU, a PAD sofreu uma diminuição ( $P<0,01$ vs $E D)$, 
mas se manteve elevada comparada ao REP ( $P<0,01$ vs REP). Na REC, o valor diminui e se assemelhou ao REP inicial $(\mathrm{P}<0,01$ vs OCLU e $\mathrm{P}=0,4$ vs REP) (Figura 7). A Tabela 9 apresenta as médias de PAD dos grupos, nas sessões PRÉ e PÓS.

Tabela 9 - Respostas de PAD $(\mathrm{mm} \mathrm{Hg})$ dos grupos durante os estágios do exercício dinâmico com "handgrip" nas sessões PRÉ e PÓS

\begin{tabular}{c|ccccc}
\hline Grupo & Sessões & Rep & ED30\% & Oclu & Rec \\
\hline SED-PLA & PRÉ & $75 \pm 8$ & $83 \pm 6$ & $79 \pm 9$ & $75 \pm 8$ \\
& PÓS & $76 \pm 8$ & $83 \pm 8$ & $80 \pm 9$ & $75 \pm 9$ \\
\hline SED-TRH & PRÉ & $76 \pm 13$ & $83 \pm 14$ & $83 \pm 14$ & $79 \pm 17$ \\
& PÓS & $74 \pm 5$ & $86 \pm 8$ & $79 \pm 8$ & $73 \pm 9$ \\
\hline TF-PLA & PRÉ & $85 \pm 11$ & $92 \pm 12$ & $87 \pm 10$ & $83 \pm 12$ \\
& PÓS & $80 \pm 10$ & $87 \pm 12$ & $85 \pm 12$ & $79 \pm 9$ \\
\hline TF-TRH & PRÉ & $82 \pm 19$ & $89 \pm 18$ & $87 \pm 18$ & $81 \pm 18$ \\
& PÓS & $77 \pm 6$ & $89 \pm 1$ & $80 \pm 7$ & $75 \pm 7$ \\
\hline
\end{tabular}

Valores em média $\pm d p$

Quando o comportamento da PAD foi comparado entre os estágios dos exercícios estático e dinâmico de todos os grupos, foi verificado que nos estágios REP, OCLU e REC os valores não apresentaram diferenças estatisticamente significante $(P=0,7, P=0,2$ e $P=0,9$ respectivamente), porém durante o EE a PAD foi significativamente maior do que $E D(P=0,04)$, conforme também demonstra a Figura 7. 
Figura 7 - Representação gráfica do comportamento da PAD durante estágios do exercício estático e dinâmico com "handgrip" de todos os grupos, nas duas sessões

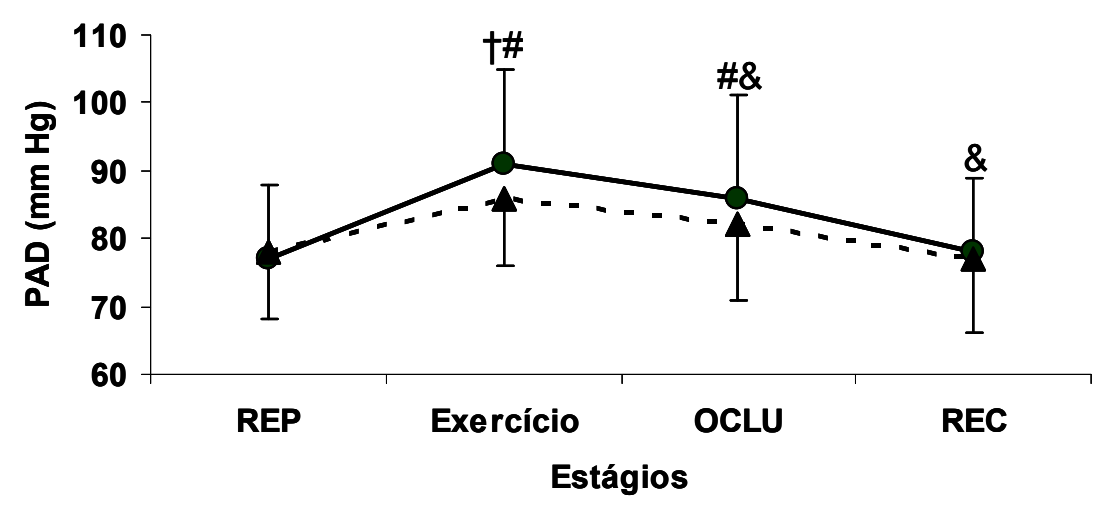

\footnotetext{
- Exercício estático ------ Exercício dinâmico

† $P<0,05$ entre estágios dos exercícios estático e dinâmico

\# $\mathrm{P}<0,05$ comparado ao estágio REP para exercícios estático e dinâmico

\& $P<0,05$ comparado ao estágio exercício para exercícios estático e dinâmico
}

\subsubsection{Pressão arterial média}

De maneira isolada, nem o TF $(P=0,3)$ nem a TRH $(P=0,9)$ influenciaram a PAM dos grupos durante a realização dos estágios do EE. A PAM aumentou na realização do EE ( $P<0,01$ vs REP). Na OCLU, a PAM diminui comparada ao EE $(P<0,01)$, porém se manteve elevada se comparada ao REP $(P<0,01)$. Na REC, a PAM diminuiu $(P<0,01$ vs $O C L U)$ e retornou a um valor semelhante ao repouso inicial ( $P=0,9$ vs REP). As médias dos quatro grupos nas duas sessões estão expostas na Tabela 10. 
Tabela 10 - Respostas de PAM $(\mathrm{mm} \mathrm{Hg})$ dos grupos durante os estágios do exercício estático com "handgrip" nas sessões PRÉ e PÓS

\begin{tabular}{c|ccccc}
\hline Grupo & Sessões & Rep & EE30\% & Oclu & Rec \\
\hline SED-PLA & PRÉ & $94 \pm 11$ & $101 \pm 7$ & $99 \pm 13$ & $92 \pm 10$ \\
& PÓS & $90 \pm 9$ & $104 \pm 13$ & $97 \pm 6$ & $88 \pm 8$ \\
\hline SED-TRH & PRÉ & $97 \pm 13$ & $112 \pm 17$ & $110 \pm 22$ & $99 \pm 15$ \\
& PÓS & $92 \pm 9$ & $106 \pm 7$ & $98 \pm 7$ & $93 \pm 7$ \\
\hline TF-PLA & PRÉ & $99 \pm 17$ & $121 \pm 15$ & $115 \pm 15$ & $102 \pm 9$ \\
& PÓS & $96 \pm 7$ & $108 \pm 8$ & $105 \pm 15$ & $97 \pm 8$ \\
\hline TF-TRH & PRÉ & $99 \pm 21$ & $106 \pm 36$ & $108 \pm 31$ & $101 \pm 20$ \\
& PÓS & $94 \pm 12$ & $107 \pm 19$ & $102 \pm 16$ & $91 \pm 11$ \\
\hline
\end{tabular}

Valores em média $\pm \mathrm{dp}$

A PAM nos estágios do exercício dinâmico não foi influenciada pelo TF $(P=0,6)$ nem pelo medicamento $(P=0,8)$ quando analisados de maneira isolada. A média da PAM não foi diferente entre sessões PRÉ e PÓS $(P=0,1)$.

Todos os grupos aumentaram a PAM durante a realização do ED ( $P<0,01$ vs REP). Na oclusão, a PAM diminui $(P<0,01$ vs $E D)$, mas se manteve mais alta do que o repouso $(\mathrm{P}<0,01$ vs $\mathrm{REP})$. Na recuperação, valor de PAM retornou ao nível do repouso $(\mathrm{P}<0,01$ vs OCLU e $\mathrm{P}=0,2$ vs REP).

Não houve diferença estatisticamente significante quando os estágios do exercício estático foram comparados ao exercício dinâmico, conforme demonstra a Figura 8. 
Tabela 11 - Respostas de PAM (mm Hg) dos grupos durante os estágios do exercício dinâmico com "handgrip" nas sessões PRÉ e PÓS

\begin{tabular}{c|ccccc}
\hline Grupo & Sessões & Rep & ED30\% & Oclu & Rec \\
\hline SED-PLA & PRÉ & $93 \pm 8$ & $101 \pm 8$ & $91 \pm 11$ & $92 \pm 7$ \\
& PÓS & $95 \pm 10$ & $100 \pm 9$ & $97 \pm 9$ & $95 \pm 11$ \\
\hline SED-TRH & PRÉ & $102 \pm 34$ & $107 \pm 36$ & $107 \pm 36$ & $101 \pm 34$ \\
& PÓS & $97 \pm 32$ & $106 \pm 35$ & $98 \pm 33$ & $97 \pm 32$ \\
\hline TF-PLA & PRÉ & $104 \pm 13$ & $109 \pm 13$ & $105 \pm 11$ & $105 \pm 12$ \\
& PÓS & $98 \pm 11$ & $106 \pm 10$ & $102 \pm 12$ & $98 \pm 10$ \\
\hline TF-TRH & PRÉ & $101 \pm 20$ & $106 \pm 23$ & $100 \pm 31$ & $97 \pm 24$ \\
& PÓS & $96 \pm 6$ & $101 \pm 14$ & $96 \pm 14$ & $92 \pm 11$ \\
\hline
\end{tabular}

Valores em média $\pm d p$

Figura 8 - Representação gráfica do comportamento da PAM durante estágios do exercício estático e dinâmico com "handgrip" de todos os grupos, nas duas sessões

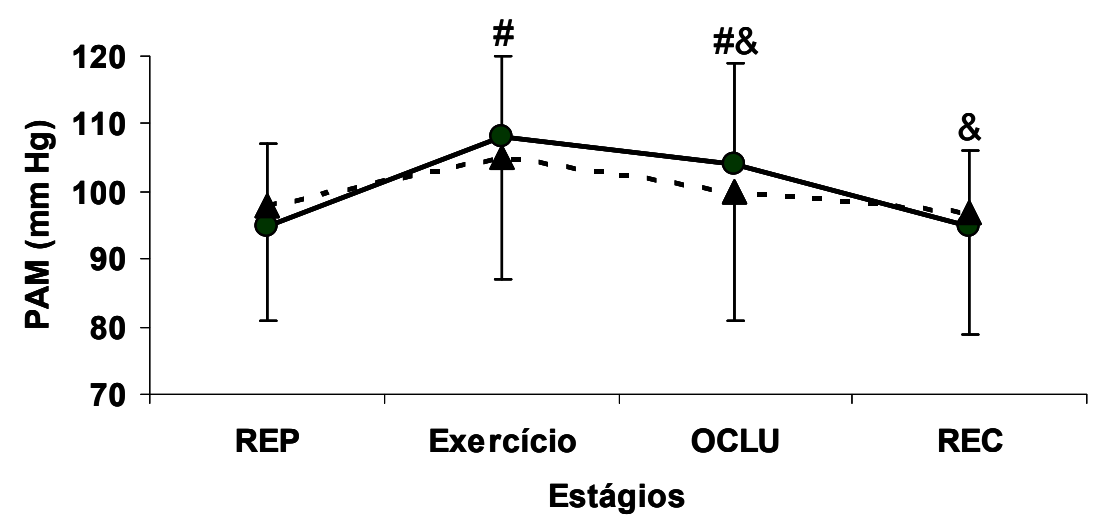

Exercício estático Exercício dinâmico

\# P $<0,05$ comparado ao estágio REP para exercício estático e dinâmico $\& \mathrm{P}<0,05$ comparado ao estágio exercício para exercício estático e dinâmico 


\subsubsection{Frequência cardíaca}

Isoladamente, nem o TF $(P=0,4)$ nem a TRH $(P=0,1)$ influenciaram a FC durante os estágios do EE. Não houve diferenças entre sessões PRÉ e PÓS $(P=0,2)$.

Houve aumento da FC durante a realização do $E E(P<0,01$ vs REP). Na oclusão, houve uma diminuição da $\mathrm{FC}(\mathrm{P}<0,01$ vs $\mathrm{EE})$, porém, ela se manteve elevada comparada ao REP $(P<0,01)$. Na recuperação, a FC voltou a valor semelhante ao REP ( $P<0,01$ vs OCLU e $\mathrm{P}=0,05$ vs REP).

Tabela 12 - Respostas de FC (bpm) dos grupos durante os estágios do exercício estático com "handgrip" nas sessões PRÉ e PÓS

\begin{tabular}{c|ccccc}
\hline Grupo & Sessões & Rep & EE30\% & Oclu & Rec \\
\hline SED-PLA & PRÉ & $69 \pm 9$ & $75 \pm 9$ & $73 \pm 8$ & $70 \pm 7$ \\
& PÓS & $69 \pm 6$ & $77 \pm 6$ & $72 \pm 7$ & $70 \pm 6$ \\
\hline SED-TRH & PRÉ & $62 \pm 11$ & $72 \pm 13$ & $66 \pm 13$ & $63 \pm 12$ \\
& PÓS & $61 \pm 11$ & $70 \pm 12$ & $61 \pm 11$ & $63 \pm 11$ \\
\hline TF-PLA & PRÉ & $66 \pm 6$ & $79 \pm 10$ & $68 \pm 6$ & $68 \pm 5$ \\
& PÓS & $64 \pm 10$ & $71 \pm 9$ & $65 \pm 7$ & $66 \pm 10$ \\
\hline TF-TRH & PRÉ & $60 \pm 9$ & $70 \pm 8$ & $66 \pm 10$ & $62 \pm 9$ \\
& PÓS & $59 \pm 8$ & $67 \pm 7$ & $63 \pm 5$ & $60 \pm 6$ \\
\hline
\end{tabular}

Valores em média $\pm d p$

Houve interação entre treinamento, medicamento e estágios, de forma que a FC do grupo SED-TRH no REP, EE, OCLU e REC foram significativamente menores $(61 \pm 11,71 \pm 13,61 \pm 12,63 \pm 11 \quad b p m$, respectivamente) do que o grupo SED-PLA $(69 \pm 7,76 \pm 7,73 \pm 7,70 \pm 6$ bpm, respectivamente), nos mesmos estágios $(P=0,01)$ (Figura 9). 
O Grupo TF-TRH apresentou FC mais baixa no REP $(59 \pm 8 b p m)$ e EE $(68 \pm 7 \mathrm{bpm})$ quando comparado ao TF-PLA $(65 \pm 8,75 \pm 10 \mathrm{bpm}$ respectivamente) $(\mathrm{P}=0,01$ para ambos) (Figura 10$)$.

Figura 9 - Representação gráfica das respostas de FC (bpm) durante os estágios do exercício estático com "handgrip" dos grupos SEDPLA e SED-TRH

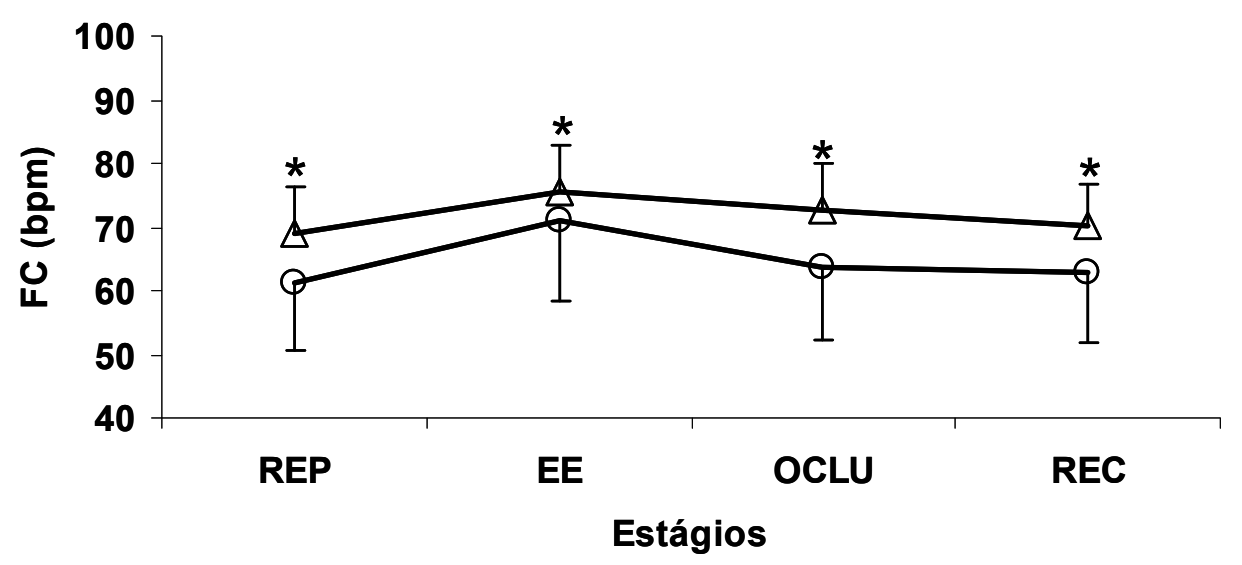

\section{$\triangle$ SED-PLA $\bigcirc$ SED-TRH $\quad{ }^{*} P<0,05$}

Figura 10 - Representação gráfica das respostas de FC (bpm) durante os estágios do exercício estático com "handgrip" dos grupos TFPLA e TF-TRH

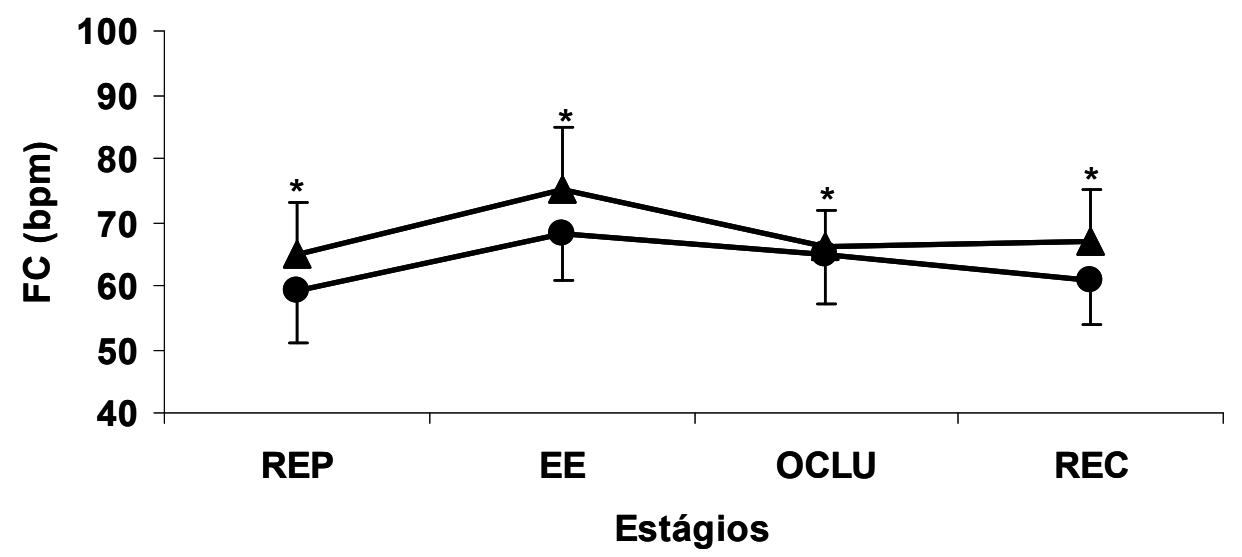

TF-PLA TF-TRH $\quad{ }^{*} P<0,05$ 
Houve um aumento da FC durante a realização do ED $(P<0,01$ vs repouso), na OCLU a FC sofreu uma redução ( $P<0,01$ vs $E E)$ atingindo valor semelhante ao REP ( $\mathrm{P}=0,4$ vs repouso).

Tabela 13 - Respostas de FC (bpm) dos grupos durante os estágios do exercício dinâmico com "handgrip" nas sessões PRÉ e PÓS

\begin{tabular}{c|ccccc}
\hline Grupo & Sessões & Rep & ED30\% & Oclu & Rec \\
\hline SED-PLA & PRÉ & $67 \pm 5$ & $74 \pm 6$ & $70 \pm 5$ & $69 \pm 4$ \\
& PÓS & $69 \pm 5$ & $73 \pm 6$ & $70 \pm 5$ & $70 \pm 6$ \\
\hline SED-TRH & PRÉ & $62 \pm 15$ & $69 \pm 13$ & $64 \pm 14$ & $63 \pm 14$ \\
& PÓS & $63 \pm 16$ & $71 \pm 11$ & $62 \pm 13$ & $60 \pm 12$ \\
\hline TF-PLA & PRÉ & $66 \pm 7$ & $75 \pm 7$ & $66 \pm 5$ & $67 \pm 7$ \\
& PÓS & $66 \pm 9$ & $73 \pm 9$ & $66 \pm 12$ & $67 \pm 11$ \\
\hline TF-TRH & PRÉ & $58 \pm 7$ & $70 \pm 9$ & $62 \pm 9$ & $59 \pm 9$ \\
& PÓS & $60 \pm 5$ & $69 \pm 7$ & $61 \pm 4$ & $59 \pm 5$ \\
\hline
\end{tabular}

Valores em média $\pm \mathrm{dp}$

Houve interação entre medicamento, sessão e estágios $(P=0,03)$, de forma que nos quatro estágios, o grupo TRH na sessão PÓS apresentou FC mais baixa (REP: $62 \pm 13$, ED: $71 \pm 10$, OCLU: $61 \pm 11$ e REC: $60 \pm 10$ bpm) comparada ao grupo PLA na mesma sessão $(68 \pm 7,73 \pm 7,68 \pm 8,69 \pm 7$ bpm, respectivamente), $(\mathrm{P}<0,01$ para todos) (Figura 11). 
Figura 11 - Representação gráfica das respostas de FC (bpm) durante estágios do exercício dinâmico com "handgrip" dos grupos PLA e TRH nas sessões PÓS

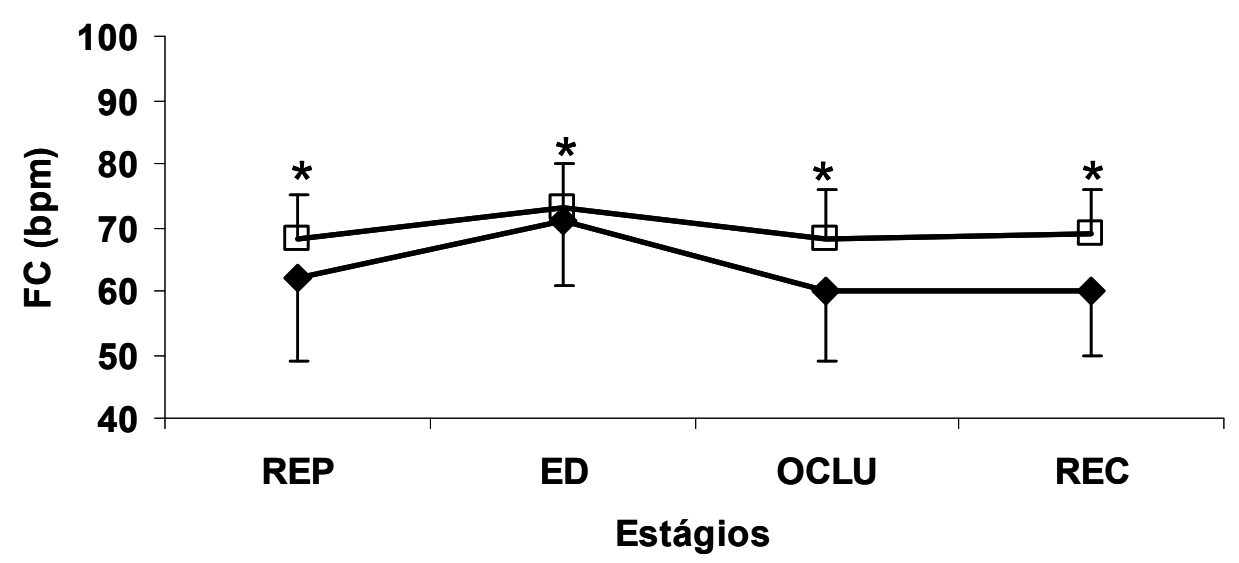

$\square$ PLA-PÓS $>$ TRH-PÓS * P<0,05

Não houve diferença estatisticamente significante entre os estágios do exercício estático e do exercício dinâmico, conforme demonstra Figura 12.

Figura 12 - Representação gráfica do comportamento da FC durante estágios do exercício estático e dinâmico com "handgrip" de todos os grupos, nas duas sessões

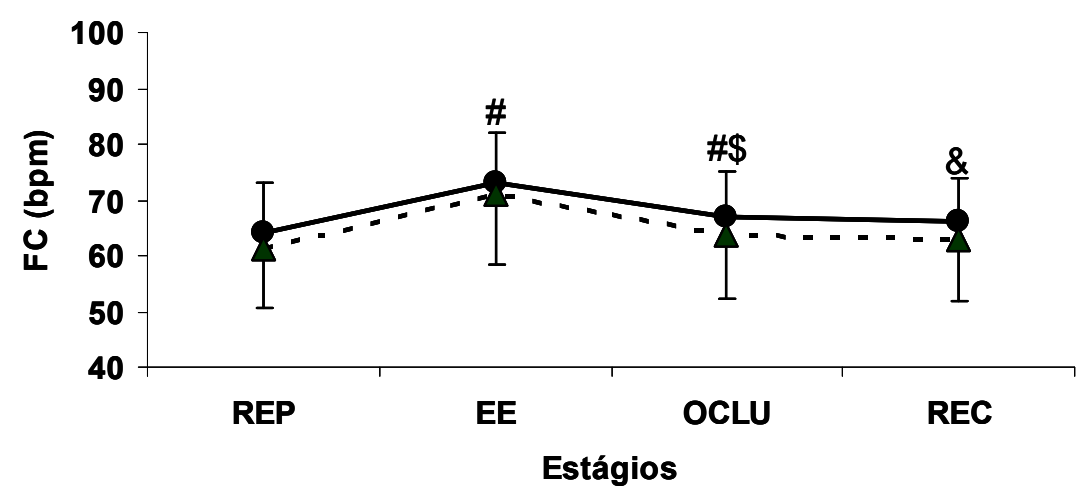

- Exercício estático ------ Exercício dinâmico

\# $\mathrm{P}<0,05$ comparado ao estágio REP para exercício estático e dinâmico

\& $\mathrm{P}<0,05$ comparado ao estágio exercício para exercício estático e dinâmico $\$ \mathrm{P}<0,05$ comparado ao estágio REP apenas para exercício estático 


\subsubsection{Fluxo sanguíneo do antebraço}

O FSA aumentou durante a realização do $\mathrm{EE}(\mathrm{P}=0,03$ vs $\mathrm{REP})$ e na oclusão diminuiu seu valor ( $\mathrm{P}=0,02$ vs $\mathrm{EE})$, assemelhando-se à recuperação ( $P=0,2$ vs $R E C)$ e ao repouso ( $P=0,9$ vs $R E P)$.

O TF $(P=0,8)$ e a TRH $(P=0,1)$ isoladamente não influenciaram as respostas de FSA nos estágios do exercício estático. A Tabela 14 demosntra as médias dos grupos, em todos os estágios, nas duas sessões.

Tabela 14 - Respostas de FSA [ml(min.100ml)] dos grupos durante os estágios do exercício estático com "handgrip" nas sessões PRÉ e PÓS

\begin{tabular}{c|ccccc}
\hline Grupo & Sessões & Rep & EE30\% & Oclu & Rec \\
\hline SED-PLA & PRÉ & $3,0 \pm 1,8$ & $3,0 \pm 2,0$ & $2,9 \pm 1,6$ & $2,6 \pm 1,4$ \\
& PÓs & $2,0 \pm 1,0$ & $2,6 \pm 1,6$ & $2,3 \pm 1,4$ & $2,1 \pm 1,4$ \\
\hline SED-TRH & PRÉ & $20, \pm 0,8$ & $2,1 \pm 1,0$ & $1,9 \pm 1,1$ & $1,7 \pm 1,0$ \\
& PÓs & $2,5 \pm 1,0$ & $2,6 \pm 1,2$ & $2,1 \pm 0,8$ & $2,0 \pm 0,6$ \\
\hline TF-PLA & PRÉ & $2,5 \pm 0,7$ & $2,9 \pm 0,4$ & $2,5 \pm 0,5$ & $2,3 \pm 0,6$ \\
& PÓS & $3,1 \pm 0,6$ & $3,7 \pm 0,5$ & $2,9 \pm 0,8$ & $2,9 \pm 0,8$ \\
\hline TF-TRH & PRÉ & $1,8 \pm 1,1$ & $2,1 \pm 0,9$ & $2,0 \pm 1,3$ & $1,8 \pm 1,0$ \\
& PÓS & $1,9 \pm 0,8$ & $2,7 \pm 1,7$ & $2,2 \pm 0,8$ & $1,5 \pm 0,6$ \\
\hline
\end{tabular}

Valores em média $\pm d p$

Nem o TF $(P=0,6)$, nem a TRH $(P=0,4)$ afetaram de maneira isolada o FSA durante os estágios do ED. Houve aumento do FSA durante a realização do $E D$ ( $P<0,01$ vs repouso), na OCLU, o FSA sofre uma redução $(P<0,01$ vs ED) e atinge valor semelhante a REC $(P=0,4)$ e REP $(P=0,7)$.

A Tabela 15 apresenta a média dos grupos, durante os estágios, nas duas sessões. 
Tabela 15 - Respostas de FSA [ml(min.100ml)] dos grupos durante os estágios do exercício dinâmico com "handgrip" nas sessões PRÉ e PÓS

\begin{tabular}{c|ccccc}
\hline Grupo & Sessões & Rep & ED30\% & Oclu & Rec \\
\hline SED-PLA & PRÉ & $2,5 \pm 1,3$ & $2,9 \pm 1,8$ & $2,9 \pm 1,9$ & $2,3 \pm 1,5$ \\
& PÓs & $2,0 \pm 0,9$ & $2,6 \pm 1,7$ & $2,0 \pm 1,3$ & $1,5 \pm 0,9$ \\
\cline { 2 - 6 } SED-TRH & PRÉ & $2,1 \pm 1,4$ & $2,5 \pm 1,6$ & $2,3 \pm 1,6$ & $2,2 \pm 1,7$ \\
& PÓS & $2,4 \pm 0,6$ & $2,5 \pm 0,3$ & $2,0 \pm 0,3$ & $1,7 \pm 0,2$ \\
\cline { 2 - 6 } TF-PLA & PRÉ & $2,7 \pm 1,0$ & $3,4 \pm 0,9$ & $2,5 \pm 0,9$ & $2,3 \pm 0,7$ \\
& PÓs & $2,7 \pm 0,6$ & $3,6 \pm 1,1$ & $2,9 \pm 1,1$ & $3,2 \pm 1,2$ \\
\cline { 2 - 6 } TF-TRH & PRÉ & $2,5 \pm 1,5$ & $2,7 \pm 1,6$ & $2,6 \pm 1,4$ & $2,1 \pm 1,3$ \\
& PÓs & $2,1 \pm 0,9$ & $2,7 \pm 1,6$ & $2,1 \pm 1,3$ & $2,4 \pm 2,1$ \\
\hline \multicolumn{7}{l}{ Valores em média $\pm d p$} \\
\end{tabular}

Não houve diferenças estatisticamente significante quando os estágios do exercício estático dos quatro grupos, nas duas sessões foram comparados com os estágios do exercício dinâmico, conforme demonstra a figura a seguir. 
Figura 13 - Representação gráfica do comportamento do FSA durante estágios do exercício estático e dinâmico com "handgrip" de todos os grupos, nas duas sessões

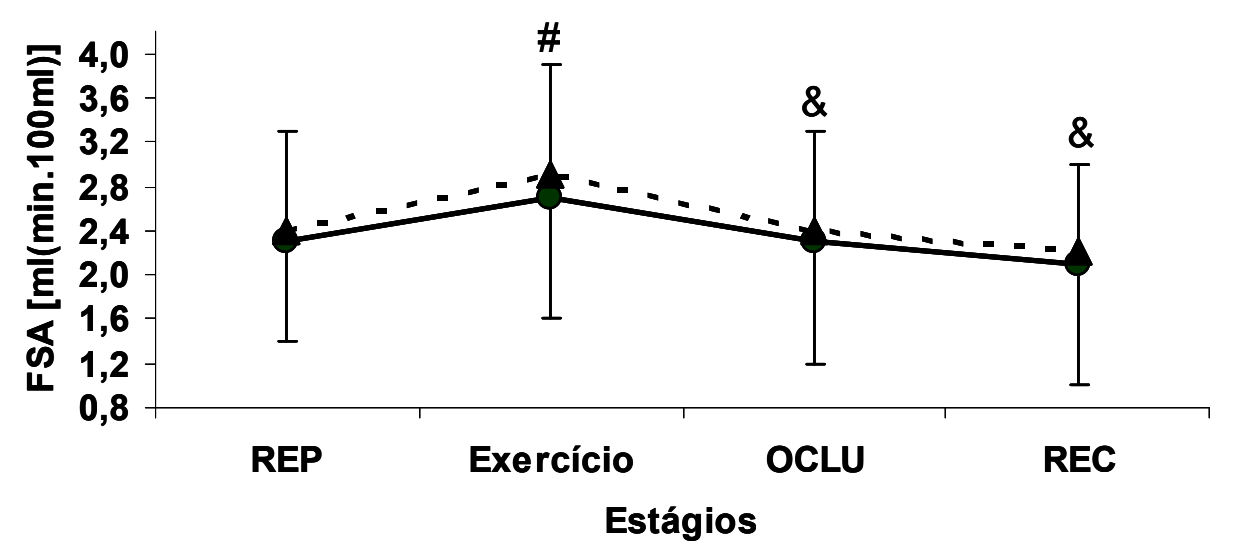

Exercício estático

- - - - Exercício dinâmico

\# $\mathrm{P}<0,05$ comparado ao repouso para exercício estático e dinâmico

\& $\mathrm{P}<0,05$ comparado ao exercício para exercício estático e dinâmico 


\section{DISCUSSÃO}

No que diz respeito à adesão das voluntárias às intervenções propostas, esta tese teve grande êxito tanto para a terapia medicamentosa (placebo e terapia hormonal) quanto para a condição (controle e treinamento aeróbico).

Em relação ao uso da terapia hormonal, a adesão à terapia medicamentosa foi completa e a efetividade foi confirmada, pois houve aumento dos níveis plasmáticos de estrogênio.

Em relação ao treinamento físico, a adesão às sessões foi superior a $90 \%$ nos dois grupos que se submeteram a esta condição. Essa adesão está foi conseguida a partir de estratégias de reposição de aulas. A efetividade do treinamento aeróbico foi comprovada através da verificação de aumento na na capacidade aeróbia dessas voluntárias.

O fato da terapia estrogênica não ter influenciado as respostas de capacidade aeróbia máxima $\left(\mathrm{VO}_{2 \text { máx }}\right)$, confome verificado em estudos prévios $^{65,66}$.

\subsection{Período Basal}

Os principais resultados encontrados no período basal deste estudo foram que o treinamento físico isoladamente:

- reduziu a ANSP e aumentou o FSA 
A terapia estrogênica associada ao treinamento físico:

- diminuiu a FC basal

A diminuição da ANSP observada neste estudo relacionada aos efeitos do treinamento físico está de acordo com outros achados da literatura, tanto em pacientes saudáveis ${ }^{79}$ quanto em indivíduos com insuficiência cardíaca $^{80}$. Embora, alguns autores tenham encontrado resultados conflitantes $^{81,82}$, a redução da ANSP após o treinamento físico parece estar relacionada ao tempo do treinamento e a massa muscular envolvida ${ }^{81}$. $\mathrm{Na}$ literatura, não existem estudos que tenham avaliado a influência do treinamento aeróbio em cicloergômetro na ANSP de mulheres pósmenopausadas. Outro estudo do nosso laboratório mostrou que nestas mulheres, após uma única sessão de exercício aeróbio em cicloergômetro, a ANSP está diminuída ${ }^{68}$. Em outros protocolos, o treinamento físico reduziu as concentrações de norepinefrina plasmática $^{83}$ e atividade simpática medida pela técnica de análise espectral ${ }^{84}$. No presente estudo também não se encontrou efeito da terapia estrogênica oral na ANSP, fato que o nosso grupo já tinha verificado após uma única sessão de exercícios aeróbios realizada depois de 6 meses de uso da terapia hormonal. Da mesma maneira, um estudo que utilizou diferentes vias de administração do estrogênio $^{8}$ encontrou reduções significativas apenas após o uso do estrogênio transdérmico, o que não ocorreu com a administração oral e que nos leva a acreditar que a via transdérmica da administração do estrogênio, diferentemente da via oral, pode influenciar a ANSP de mulheres no 
climatério. A explicação para isso é que quando o estrogênio é administrado oralmente, é rapidamente hidrolisado em estradiol durante sua primeira passagem pelo fígado. O estradiol é convertido em estrona, que é uma forma menos potente do hormônio ${ }^{85}$. Essa passagem hepática expõe o fígado a uma dose supra fisiológica de estrogênio, que resulta numa diminuição na síntese de fator de crescimento de insulina (IGF-1) ${ }^{86}$. A administração de IGF-1 em ratos diminuiu a atividade nervosa simpática ${ }^{87}$ enquanto que a deficiência deste hormônio é associada à hiperatividade simpática em humanos ${ }^{88}$.

No período basal, também foi observado que o treinamento físico não alterou as pressões sistólica, diastólica e média das mulheres estudadas. $\mathrm{O}$ efeito do treinamento físico na pressão arterial já foi amplamente estudado e o exercício é parte do tratamento não medicamentoso para hipertensos. Pescatello et. al., (2004) ${ }^{89}$ verificaram que a maior redução na pressão arterial ocorre quanto maiores forem os níveis iniciais. Neste sentido, uma revisão sistemática dos estudos randomizados e controlados até 2004 sobre exercício em mulheres pós-menopausadas ${ }^{57}$ concluiu que as mulheres que mais se beneficiaram da redução da pressão arterial com o treinamento físico foram as hipertensas e obesas, diferente das voluntárias do presente estudo o que pode justificar nossos achados de ausência de redução na pressão arterial.

A FC foi significativamente menor na sessão PÓS quando comparada a sessão PRÉ de todos os grupos, porém, quando os grupos foram analisados separadamente, o grupo TF-TRH foi o único que apresentou 
diferença estatisticamente significante. Dessa forma, pode-se acreditar que a terapia hormonal associada ao treinamento físico reduz a FC. De maneira isolada, alguns estudos observaram redução da FC com o treinamento físico e tal fato parece estar relacionado a uma alteração na modulação autonômica cardiovascular promovida pelo exercício ${ }^{90}$, porém esses resultados não são unânimes ${ }^{79}$. Em mulheres pós-menopausadas, Cardoso Jr. $(2009)^{91}$ não verificou modificações na modulação autonômica de mulheres climatéricas com o treinamento físico. Por outro lado, tanto a terapia hormonal oral como parenteral parecem exercer influência sobre esta variável $^{8}$. Estudos com análise espectral ${ }^{92}$, mostraram que mulheres que utilizaram estrogênio transdérmico por 4 meses obtiveram maior duração do intervalo R-R. Há também evidências da atenuação da variabilidade da freqüência cardíaca após a utilização de estrogênio combinado com progestagênio $^{93}$, assim como a ooforectomia diminuiu a atividade parassimpática e aumentou a predominância simpática com a retirada dos hormônios ovarianos ${ }^{94}$. Portanto, estes estudos sugerem que a terapia hormonal modula a hiperatividade simpática para o coração, reduzindo a FC. Dessa forma, no presente estudo, não se pode atribuir a redução da FC ao treinamento ou estrogênio de maneira isolada, porém, observou-se que a associação das intervenções contribuiu para a redução desta variável, o que implica em um benefício cardiovascular para essa população ${ }^{95}$.

Outro achado importante de nosso estudo foi o fato de o treinamento físico ter propiciado um aumento no FSA. De fato, há evidências que uma única sessão de exercícios promove vasodilatação ${ }^{68,96}$ e diminuição da 
resistência vascular periférica ${ }^{96}$ de mulheres pós-menopausadas, assim como o treinamento físico realizado periodicamente ${ }^{97}$. É possível que o aumento do FSA esteja intimamente relacionado à redução da ANSP observada. Outros fatores que podem estar implicados são a liberação de fatores relaxantes do endotélio como o óxido nítrico, prostaciclina, fator hiperpolarizante do endotélio e prostaglandina E, além da redução da ação de agentes vasoconstritores endoteliais como endotelina-1, angiotensina e tromboxano ${ }^{98}$.

Pelos efeitos vasculares positivos do estrogênio evidenciados em inúmeros estudos ${ }^{99-101}$, esperava-se que a terapia hormonal exercesse alguma influência no FSA das pacientes, mas isso não ocorreu. Recentemente quando se utilizou a mesma dose do hormônio não foi observada melhora no fluxo sanguíneo de repouso após até 3 meses do uso do hormônio ${ }^{102}$. Aparentemente, os benefícios vasculares do estrogênio são mais bem evidenciados em mulheres que iniciam a terapia mais jovem e, principalmente logo após a menopausa ${ }^{103}$. Em nosso estudo não foi possível determinar com exatidão a época da menopausa, porém, a média de tempo desde a histerectomia foi de $8 \pm 6$ anos, o que pode ter implicado em prejuízo da resposta vasodilatadora nestas mulheres.

Desta forma, os achados do presente estudo demonstraram que a terapia hormonal associada ao treinamento físico reduziu a FC de repouso, e o treinamento físico aeróbio reduziu a ANSP e aumentou o FSA. Todos esses fatores contribuem para redução do risco cardiovascular de mulheres pos-menopausadas, histerectomizadas e saudáveis. 


\subsection{Exercícios estático e dinâmico com "handgrip"}

A execução do exercício estático com "handgrip" provocou aumento das seguintes variáveis nos 4 grupos:

- ANSP, PAS, PAM que se mantiveram elevadas até a oclusão;

- $\mathrm{PAD}, \mathrm{FC}$ e FSA que, no período de oclusão, sofreram reduções

A execução do exercício dinâmico com "handgrip" provocou aumento das seguintes variáveis nos 4 grupos:

- ANSP que se manteve elevada na oclusão e na recuperação;

- PAD e FC que na oclusão diminuíram seus valores;

- PAS e PAM, que se mantiveram elevadas na oclusão;

- FSA que na oclusão retornou a valor igual ao inicial.

O treinamento físico isolado não alterou as respostas de nenhuma das variáveis estudadas.

A terapia estrogênica de maneira isolada diminuiu a FC nos estágios: repouso, exercício estático, oclusão e recuperação do grupo sedentário;

A terapia estrogênica associada ou não ao treinamento aeróbio reduziu a FC nos estágios repouso, exercício dinâmico, oclusão e recuperação, quando comparado ao placebo.

A terapia estrogênica associada ao treinamento aeróbio diminuiu a FC nos estágios repouso e exercício estático. 
Os exercícios estáticos e dinâmicos com "handgrip" são utilizados no intuito de testar a alça eferente simpática do reflexo pressorreceptor, como mencionado anteriormente. Os ajustes autonômicos conseqüentes à realização do exercício estático decorrem de dois mecanismos que interagem entre si: o comando central e aferentes musculares. $O$ circuito neural do comando central parece englobar regiões do córtex motor que interagem com estruturas talâmicas e cerebrais de integração cardiovascular ${ }^{104}$. O comando central é responsável principalmente pelas respostas de FC ao exercício. Ela se eleva imediatamente após o inicio do exercício $^{105,106}$ e essa resposta cronotrópica se relaciona com a redução da atividade parassimpática. No início do exercício, o comando central também inibe a ANSP que vai se elevar apenas após o segundo minuto da manobra excitatória e durante a oclusão da circulação da musculatura exercitada. Neste momento o comando central retira sua ação e diminui a FC e a ANSP permanece aumentada principalmente pela ação metabólica ${ }^{105}$. A percepção de metabólitos por receptores sensoriais sensíveis promove o segundo mecanismo de ajuste autonômico: a resposta de pressão arterial reflexa decorrente da contração muscular sustentada. Essa ativação das aferências musculares quimiossensíveis parece ser o principal estímulo para a intensificação da ANSP ${ }^{105,107 .}$

Durante o exercício dinâmico com "handgrip", além da ativação dos quimiorreceptores, há uma ativação dos mecanorreceptores, que também são responsáveis por deflagrar as respostas hemodinâmicas e neurais ${ }^{106}$. 
A oclusão da circulação sanguínea do braço exercitado é realizada após as duas formas de exercício (estático e dinâmico) com "handgrip" no intuito de manter a ativação dos aferentes musculares quimiossensíveis ${ }^{105}$. Com esta manobra, se mantém o estímulo pela ativação do quimiorreflexo na ausência de outra influência excitatória como aquelas presentes durante o exercício (comando central e os reflexos mecânicos musculares) ${ }^{108}$.

No presente estudo, a realização de 3 minutos de contração estática e dinâmica, promoveu elevação de todas as variáveis estudadas (ANSP, PAS, PAD, PAM, FC e FSA), conforme observado em estudos prévios ${ }^{108-110}$

No momento da oclusão da circulação do braço após o exercício estático, as variáveis sofrem uma atenuação, porém se mantêm elevadas quando comparadas ao repouso. Esses achados também estão de acordo com a literatura, quando outras populações foram avaliadas ${ }^{73,111}$.

Após o exercício dinâmico, o que verificamos foi que a oclusão da circulação do braço promoveu a redução de PAD, FC e FSA enquanto que ANSP, PAS e PAM se mantiveram elevadas. Além do estímulo químico liberado pela a realização dos exercícios, acredita-se que a oclusão promove uma dor local, o que pode contribuir para ANSP se manter elevada neste período, uma vez que em outro estudo, houve aumento da ANSP na oclusão, mesmo após intensidades baixas de exercício dinâmico em que a ANSP não se alterou ${ }^{109 .}$

Estudos que compararam as respostas ao exercício estático e dinâmico em indivíduos saudáveis verificou-se um maior aumento de pressão arterial durante o exercício estático e um maior aumento de FC 
durante o exercício dinâmico ${ }^{112,113}$. Isso ocorre uma vez que a estimulação química a partir dos metabólitos liberados no exercício estático promove uma resposta excitatória maior do que a estimulação mecânica gerada pelos exercícios dinâmicos ${ }^{114}$. No presente estudo, quando comparamos os valores das variáveis obtidas nos exercícios estático e dinâmico, observamos que os dois tipos de exercícios promovem resposta semelhante de PAS, PAM, FC e FSA. Apenas a ANSP e PAD foram significativamente maiores durante a realização do exercício estático. Tal fato demonstra que para a população estudada, o exercício estático parece promover respostas exacerbadas relacionadas à vasoconstrição periférica.

Sabe-se que com o envelhecimento, o comportamento da pressão arterial e da ANSP são atenuadas frente a estímulos estáticos ${ }^{115,}{ }^{116}$ e dinâmicos ${ }^{115}$. Acredita-se que com o envelhecimento, as células musculares se tornam mais oxidativas e por isso produzem uma menor resposta de $\mathrm{H}^{+} \mathrm{O}$ que promove menor elevação de pressão arterial e ANSP, comparativamente a músculos glicolíticos que geram uma resposta aumentada.

Um fato que deve ser levado em conta é que no presente estudo a medida da ANSP foi realizada no nervo fibular da perna cuja musculatura não foi exercitada durante a realização das manobras excitatórias. Essa medida reflete a ação do sistema nervoso simpático naquele local que não necessariamente é a mesma em outros leitos. Com relação à musculatura envolvida no exercício, acredita-se que os metabólitos musculares resultantes da contração muscular causem diminuição da resposta 
vasoconstritora $^{117,118}$. Esse mecanismo otimiza o fluxo sanguíneo em face ao aumento da vasoconstrição simpática que ocorre durante o exercício ${ }^{119} \mathrm{e}$ é conhecido como simpatólise funcional ${ }^{120}$. Estudos prévios verificaram diminuição da simpatólise funcional com o avanço da idade em homens ${ }^{121} \mathrm{e}$ então ocorre uma maior vasoconstrição local mediada pelo sistema nervoso simpático. Posteriormente, Fadel el al., $(2004)^{119}$ verificaram responsividade simpática aumentada nos músculos exercitados em mulheres com deficiência estrogênica que foi revertida com 1 mês de reposição hormonal transdérmica em mulheres pós-menopausadas. Os possíveis mecanismos envolvidos na maior simpatólise funcional na presença do estrogênio parecem ser maior expressão de óxido nítrico, ativação dos canais de potássio e diminuição do estresse oxidativo ${ }^{119}$. Não foi nosso objetivo avaliar a resposta local aos exercícios estático e dinâmico, por isso não avaliamos possíveis alterações na simpatólise funcional, porém pode-se especular que apesar de não termos observado respostas atenuadas de ANSP medida na perna, de alguma forma a terapia estrogênica pode ter contribuído para o aumento da simpatólise funcional do antebraço exercitado.

Ao se avaliar e comparar os efeitos isolados e associados da reposição hormonal oral estrogênica e do exercício físico aeróbio nas respostas hemodinâmicas e neurais ao exercício estático e dinâmico com "handgrip" pode-se verificar que o treinamento físico isoladamente não alterou as respostas hemodinâmicas e neurais frente ao exercício estático e nem ao exercício dinâmico, com exceção da FC. Esse achado corrobora com outro estudo $^{110}$ que atribuiu esses resultados ao tipo de exercício realizado que foi 
o aeróbio. É possível que outro tipo de treinamento como exercícios de força pudesse promover adaptações cardiovasculares diferentes que resultariam em respostas ao estresse ${ }^{110}$. Além disso, no presente estudo a massa muscular envolvida no treinamento foi predominantemente dos membros inferiores, enquanto que para a realização dos exercícios com "handgrip" usou-se o braço.

Os achados dos efeitos da terapia estrogênica isolada nas respostas de FC nos exercícios estático e dinâmico reforçam os conceitos já discutidos anteriormente de que o estrogênio possivelmente reverte o déficit vagal e a hiperatividade simpática ${ }^{122,123}$. A partir de nossos achados, acredita-se que essa modulação simpática central promovida pelo estrogênio reduza a FC no repouso e em situações de estresse fisiológico como durante a realização dos exercícios estático e dinâmico com "handgrip".

Quando a terapia estrogênica foi associada ao treinamento físico verificou-se atenuação das respostas de FC nos 4 estágios do exercício dinâmico, e nos estágios repouso e exercício estático. Esses achados nos levam a crer que o treinamento físico aeróbio, pode ter contribuído para menores respostas de FC nos estágios de oclusão e recuperação após exercício dinâmico, que não foram observadas após o exercício estático.

No que diz respeito à ação do estrogênio às respostas de ANSP, PAS e PAM aos exercícios com "handgrip", estudos prévios nos faziam acreditar na hipótese de que o estrogênio reduziria o reflexo pressor. Quando homens e mulheres entre 32 a 43 anos foram avaliados durante o exercício estático, observou-se que as mulheres apresentaram menor elevação de pressão 
arterial e da ANSP do que os homens ${ }^{124}$. Os mesmos autores, posteriormente, avaliaram mulheres em diferentes fases do ciclo menstrual ${ }^{111}$ e verificaram menores respostas quando as taxas de estrogênio estavam aumentadas e dessa forma, acreditou-se que o estrogênio agiria no sistema nervoso autônomo, impedindo aumentos exacerbados de pressão arterial e ANSP frente ao estresse fisiológico. Entretanto, estudos em animais apesar de terem observado influências positivas da ação do estrogênio, possuíam algumas ressalvas que nos ajudam a explicar nossos achados com relação à pressão arterial e ANSP. Um deles observou que injeção intravenosa de estrogênio atenuava o comando central, porém não alterava o reflexo pressor $^{125}$. Outro protocolo verificou que a aplicação de $17 \beta$ estradiol na medula espinhal de gatos atenuou o reflexo pressor apenas quando as concentrações do hormônio excediam as taxas fisiológicas ${ }^{126}$. Da mesma forma, Schmitt \& Kaufman, $2003^{127}$ observaram que estrogênio injetado na medula de gatos de ambos os sexos promovia atenuação do reflexo pressor em todos apenas em doses mais altas. É possível que em nosso estudo a atenuação do reflexo pressor não tenha sido observada durante os exercícios estático e dinâmico principalmente devido a dose de estrogênio empregada no protocolo. Sudhir, et al., (1997) ${ }^{128}$ não realizaram manobras com "handgrip", porém verificaram atenuação as respostas vasoconstritoras à norepinefrina e redução do "spillover" de norepinefrina em mulheres pós-menopausadas que utilizaram $2 \mathrm{mg}$ de valerato de estradiol por 8 semanas, nos levando a crer que $1 \mathrm{mg}$ de valerato de estradiol seja uma dose ineficiente para tal ação. Uma outra explicação para nossos 
achados está relacionada à participação dos ovários na atenuação do reflexo pressor. Um estudo com gatas verificou que o reflexo pressor era atenuado de maneira mais expressiva nos animais que tinham os ovários intactos, quando comparado às ooforectomizadas ${ }^{129}$. Em nosso estudo em mulheres histerectomizadas apesar da grande maioria das voluntárias não serem também ooforectomizadas seus ovários não tinham suas funções normais.

Por fim, acreditava-se que a associação da terapia estrogênica com o treinamento físico promovesse respostas potencializadas de redução de ANSP, pressão arterial e aumento de FSA, que contribuiriam para a prevenção de risco cardiovascular dessas mulheres tanto no período basal, comparável a uma situação de repouso, quanto durante as manobras de com "handgrip" que poderiam representar esforços físicos realizados pelas mulheres em seu dia-a-dia. No entanto esses efeito não foram verificados. Esses resultados podem estar relacionados ao tipo, dose e forma de administração do hormônio utilizado. Por isso novos estudos envolvendo outras formas de terapia se fazem necessários.

\subsection{Dificuldades e limitações do estudo}

A primeira grande dificuldade desse estudo foi encontrar voluntárias que preenchessem os critérios de inclusão, uma vez que é muito comum nessa faixa etária a presença de co-morbidades associadas ao estado pós-menopáusico. 
A segunda dificuldade foi realizar as duas sessões experimentais do estudo. Como esse trabalho é uma alça de um projeto temático, que envolve inúmeros exames, houve voluntárias que, ao chegar ao sexto mês de seguimento, se negaram a realizar mais sessões experimentais e acabaram abandonando o estudo.

Além do elevado número de exames a que as voluntárias eram submetidas, a microneurografia é uma técnica invasiva que pode provocar desconforto nas pacientes, fato que fez com que algumas voluntárias se recusassem a realizar a segunda sessão.

A microneurografia é uma técnica de difícil execução e muitas vezes, apesar de o nervo ser localizado, não se conseguiu registros adequados para analise o que levava à interrupção da sessão experimental e a voluntária era orientada a retornar após 15 dias para uma nova tentativa. Caso na segunda tentativa não fosse possível novamente acessar um bom sinal nervoso, a sessão era realizada sem os dados da microneurografia.

Outra dificuldade que tivemos foi para se coletar registros do fluxo sanguíneo do antebraço. Muitos dados foram perdidos em função de problemas técnicos com o plestismógrafo e também, durante a realização dos exercícios estáticos e dinâmicos com "handgrip", as voluntárias movimentavam o braço em que o registro do fluxo estava sendo realizado, prejudicando a leitura de vários traçados.

Dessa forma, para não prejudicar o andamento do trabalho, muitas sessões foram realizadas e os dados de ANSP e FSA foram desconsiderados. Porém, quando a análise estatística foi realizada, foi 
necessário desconsiderar os dados das duas sessões daquela paciente que possuía algum dado ausente, reduzindo, consideravelmente nossa amostra para análise.

E apesar de inúmeros esforços na tentativa de aumentar o número de voluntárias, como por exemplo: anúncios em jornais (Folha de São Paulo, Diário de São Paulo, Estado de São Paulo, Jornal do Bairro - região do Butantã) e rádios (CBN, Bandeirantes e USP); afixação de cartazes em pontos de ônibus do Campus Universitário da Universidade de São Paulo, na e na Companhia Paulista de Trens Metropolitanos (CPTM), não foi possível um numero mais expressivo de voluntárias que concluíssem o estudo em tempo hábil para a finalização desta tese.

Apesar de o protocolo experimental adotado neste estudo ter sido o mesmo de outros estudos de nosso laboratório, uma limitação refere-se à medida da pressão arterial durante as sessões experimentais que foi realizada na perna. Sabe-se que esta medida aumenta os valores da pressão arterial sistólica em aproximadamente $30 \mathrm{~mm} \mathrm{Hg}^{130}$, o que compromete a comparação dos níveis de pressão arterial sistólica obtidos nesta tese com os níveis observados em outros estudos que realizaram a medida no braço. Porém como todas as medidas foram realizadas em todos os grupos, nas mesmas condições, o local de medição provalemente não influenciou os resultados encontrados.

A pressão arterial foi medida na perna devido ao fato de que durante os experimentos, um dos braços estava sendo utilizado para a 
medida do fluxo sanguíneo do antebraço e com o outro braço a voluntária executava as manobras excitatórias de exercícios com "handgrip". 


\section{CONCLUSÃO}

Nesta tese, verificou-se que, em mulheres histerectomizadas, saudáveis e na pós-menopausa, a terapia oral estrogênica de $1 \mathrm{mg} / \mathrm{dia}$ de valerato de estradiol e o treinamento físico aeróbio em cicloergômetro promovem de maneira isolada ou associada alterações hemodinâmicas e neurais que podem contribuir para redução do risco cardiovascular.

De maneira isolada a terapia estrogênica reduziu a freqüência cardíaca nos estágios do exercício estático e dinâmico com "handgrip".

O treinamento físico isoladamente diminuiu a atividade nervosa simpática periférica e aumentou o fluxo sanguíneo do antebraço no período basal.

A associação da terapia estrogênica com o treinamento físico reduziu a FC no período basal, em todos os estágios do exercício dinâmico e nos estágios de repouso e exercício estático com "handgrip". 
7 ANEXOS

ANEXO A

Aprovações do Comitê de Ética

\author{
HOSPITAL DAS CLINICAS \\ t: :

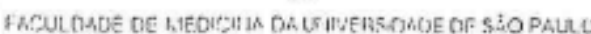 \\ CAIYA POSIAL, gOP? \\ sío ravo- Rans: \\ DIRETORIA CLÍNICA
}

\title{
Comissão de Ética para Análise de Projetos de Pesquisa
}

\section{APROVACẼO}

A Comissão de Ético paro Análise de Projetos de Pesquisa -

CAppesq da Diretoria Clinica do Hospital das Clínicos e da Faculdade de Medicina da Universidade de 50̆ Poulo, em sessão de 14.11.01. APROVOU o Protocolo de Pesquisa $n^{\circ}$ CO2/OI, intitulado: "Sistema nervoso simpático, hipertensão orterial e condicionamento físico em mulheres pós-menopausadas recebenda Teropia de Reposicgão Hormonal", da Departamento de Obstetricia e Ginecologio, bem como o Termo de Consentimento Livre e Esclarecido, Ressaltamos a necessidade de considerar as ressalvas do Departamento de Radiologia mencionodas a seguir:

- Os exomes de mamografia e densitometria óssea executados no Instituto de Radiologia pela sua quantidade näo deveräa interferir na rotina do serviço.

- Considerando os aspectes śticos decorrentes do relação médico-paciente seria necessário o participoção de profissionais nossos, capazes assim, de fazer um elo entre pacientes e pesquisodores, facilitondo o desenvolvimento da pesquiso.

- Os profissionais lembrados para este fim são: Dr. Aurélio Boreli (Densitonetria) e Dr. Nestor de Barros.

Pesquisadora Responsável: Profa, Ora. Ângela Maggio da Fonseca

Pesquisador Executante: Dr. Fabricio Collares Rosas

CAPPesq, 16 de novembro de 2001.

PROF. DR. JORGE KALIL FILHO

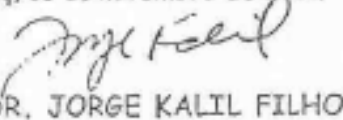

Presidente da Comissão Ética para Análise de Projetos de Pesquisa

OBSERVAÇÃO: Cabe co pesquisodor elaborar e apresentar à CAPPesq, os relatórios parciais e final sobre a pesquiso (Resolução do Conselho Nacional de Saúde $n^{\circ} 196$, de 10.10 .1996 , inciso IX,2, letra " $\mathrm{c}$ )

continua 


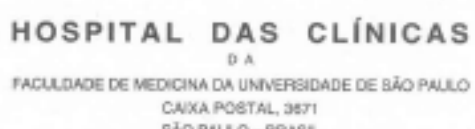

Comissão de Ética para Análise de Projetos de Pesquisa

\section{APROVACẼO}

A Comissão de Ética para Análise de Projetos de Pesquisa - CAPPesq da Diretoria Clínica do Hospital das Clínicas e da Faculdade de Medicina da Universidade de Săo Paulo, em sessão de 12.06.02, APROVOU os documentos abaixo mencionados referente ao Protocolo de Pesquisa $n^{\circ} 002 / 01$, intitulado: "Impacto isolado e associado do exercício físico e da reposição estrogênica em mulheres climatéricas", apresentado pelo Departamento de OBSTETRÍCIA E GINECOLOGIA.

- Emenda versão datada de 19.04.02

- Protocolo de Pesquisa Emendado

- Novo Termo de Consentimento Livre e Esclarecido

- Pesquisadora Responsável: Profa. Dra. Ângela Maggio da Fonseca

- Pesquisador Executante: Dr. Fabrício Collares Rosas

CAPPesq, 12 de Junho de 2002.

PROF. DR. JORGE KALIL FILHO Presidente da Comissäo Ética para Análise de Projetos de Pesquisa

OBSERVAÇÃO: Cabe ao pesquisador elabarar e apresentar d CAPPesq, os relatórios parciais e final sobre a pesquisa (Resoluçäo do Consel/ho Nacional de Saúde $n^{\circ}$ 196, de 10.10.1996, inciso IX.2, letra "c"). 
ANEXO B

Termo de Consentimento Livre e Esclarecido

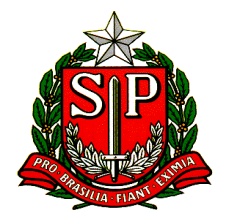

\section{HOSPITAL DAS CLÍNICAS}

da Faculdade de MEdicina da UNIVERSIDADE dE SÃo PaUlo

CaIXa Postal, 8091 - SÃo PaUlo - BRAsil

I - DADOS DE IDENTIFICAÇÃO DO SUJEITO DA PESQUISA OU RESPONSÁVEL LEGAL

1. NOME DO PACIENTE .

DOCUMENTO DE IDENTIDADE $\mathrm{N}^{\circ}$ SEXO : $M \quad F$ DATA NASCIMENTO: $. / \ldots \ldots . . . . . . .$. $\mathrm{N}^{0}$ APTO:

ENDEREÇO CIDADE

BAIRRO: TELEFONE: DDD

CEP: ..)

2.RESPONSÁVEL LEGAL

NATUREZA (grau de parentesco, tutor, curador etc.)

DOCUMENTO DE IDENTIDADE: SEXO: $M \quad F$

DATA NASCIMENTO: I........... APTO:

ENDEREÇO: $\mathrm{N}^{0}$ CIDADE: BAIRRO:

CEP: TELEFONE: DDD

\section{II - DADOS SOBRE A PESQUISA CIENTÍFICA}

1. TÍTULO DO PROTOCOLO DE PESQUISA:

"Impacto isolado e associado do exercício físico e da terapia de reposição estrogênica em mulheres climatéricas".

PESQUISADOR: Profa Dra Ângela Maggio da Fonseca CARGO/FUNÇÃO: Professora Associada INSCRIÇÃO CONSELHO REGIONAL Nº UNIDADE DO HCFMUSP: Departamento de Ginecologia e Obstetrícia 
2. AVALIAÇÃO DO RISCO DA PESQUISA:

$\begin{array}{lll}\text { SEM RISCO } & \text { RISCO MÍNIMO } & \text { RISCO MÉDIO x } \\ \text { RISCO BAIXO } & \text { RISCO MAIOR } & \end{array}$

(probabilidade de que o indivíduo sofra algum dano como consequência imediata ou tardia do estudo)

3. DURAÇÃO DA PESQUISA : 4 anos

\section{III - REGISTRO DAS EXPLICAÇÕES DO PESQUISADOR AO PACIENTE OU SEU REPRESENTANTE LEGAL SOBRE A PESQUISA CONSIGNANDO:}

\section{Justificativa e os objetivos da pesquisa;}

A senhora está sendo convidada a participar de uma pesquisa que irá avaliar os efeitos isolados e combinados do estrogênio, que é o hormônio usado para o tratamento da menopausa, e do exercício físico sobre o coração, os fatores de risco cardiovascular, que aumentam o risco de ataque cardíaco, e a qualidade de vida, que vem a ser como a senhora percebe os benefícios do tratamento sobre o seu dia a dia.

\section{Procedimentos que serão utilizados e propósitos, incluindo a identificação dos procedimentos que são experimentais;}

O medicamento que será utilizado é um hormônio chamado valerato de estradiol e é vendido nas farmácias com o nome de PRIMOGYNA. A senhora será acompanhada no ambulatório da ginecologia pelo prazo de 12 meses, que é o tempo previsto para a duração da pesquisa. Nesse período a senhora deverá comparecer a todas as consultas agendadas e deverá fazer os exames recomendados.

A seqüência da pesquisa será a seguinte:

1) Uma consulta inicial com o ginecologista, quando será feito um check up geral. Ele irá examiná-la, avaliará os sintomas da menopausa e medirá sua pressão. Serão feitos exames de sangue para verificar se a senhora é diabética, se tem colesterol e outras gorduras altas no organismo, como estão os seus hormônios, se o seu fígado e os seus rins funcionam adequadamente. Além disso, serão feitos a densitometria óssea para verificar se a senhora tem osteoporose da menopausa, a mamografia para ver se há algum caroço nos seus seios e uma ultra-sonografia para verificar como estão os seus órgãos internos. Todos esses exames são de rotina no tratamento da menopausa;

2) Um teste com uma bicicleta ergométrica (ergoespirométrico) onde a senhora irá pedalar em uma velocidade cada vez maior, até o máximo que a senhora conseguir. Ao final, é normal sentir-se cansada. Esse teste serve para avaliar a sua capacidade física máxima, além de poder detectar algum problema cardíaco. Ele poderá ser interrompido a qualquer momento, 
bastando avisar que deseja parar. O resultado desse teste será usado para orientar o treinamento físico que algumas das senhoras serão sorteadas para fazer;

3) uma avaliação da sua pressão arterial por 24 horas (MAPA). Nesse exame será colocado um aparelhinho que mede a pressão automaticamente. É semelhante a um walkman, que fica preso a cintura e é ligado a uma bolsa de medir a pressão no braço. Ele é capaz de medir a sua pressão de tempos em tempos, durante o dia e a noite inteiros. Por esse motivo é importante que a senhora mantenha as suas atividades habituais quando estiver com o aparelhinho. Através desse exame será possível verificar o comportamento da sua pressão durante todo um dia. Algumas pessoas queixam-se de dormirem mal com ele;

4) um exame que irá avaliar se a senhora tem tendência para o diabetes (clampeamento euglicêmico). Nesse exame a senhora receberá em uma veia insulina, que é o hormônio que falta aos diabéticos, e soro com glicose pelo período de 2 horas. Enquanto a senhora estiver tomando esse soro serão colhidos alguns exames de sangue para verificar o nível de açúcar. Durante todo o exame será medido o fluxo sangüíneo no outro braço, através de um aparelho igual ao de medir a pressão, que será inflado e desinflado automaticamente de tempos em tempos. Seu braço ficará apoiado em suporte para facilitar as medidas;

5) um exame que irá verificar como o seu sistema nervoso controla a sua circulação (microneurografia). Nesse exame serão colocadas 2 agulhinhas bem fininhas na sua perna, que estará apoiada em uma almofada. Durante esse exame a senhora irá fazer um exercício com a mão, apertando e segurando firme um aparelho semelhante a uma alça de mala. Esse exercício será feito também apertando e soltando essa alça, seguindo um ritmo predeterminado. Além disso, terá que dizer rapidamente qual a cor da tinta que está escrito o nome das cores em uma folha de papel;

6) um questionário onde responderá a umas perguntas, antes e depois do tratamento com o hormônio e do treinamento físico, para saber se, na sua opinião, o seu dia a dia melhorou ou não.

Todas as senhoras serão sorteadas no início da pesquisa. Esse sorteio servirá para separá-las em 2 grupos: um grupo receberá hormônio e o outro receberá um medicamento sem princípio ativo (placebo). Nem a senhora e nem os médicos saberão a que grupo a senhora irá pertencer durante toda a pesquisa. Isso só será revelado no final.

Da mesma forma, serão sorteadas aquelas que farão ou não o treinamento físico. Aquelas que participarem do grupo de treinamento farão três sessões semanais de exercício e as sorteadas para o outro grupo serão solicitadas a repetir os exames de clampeamento e da microneurografia, após uma única sessão de exercício na bicicleta ergométrica.

Todas as senhoras terão consultas com o ginecologista a cada 2 meses para verificar sua pressão arterial e o seu peso, além do exame ginecológico de rotina. Os exames de sangue e os de rotina da menopausa serão repetidos no $6^{\circ}$ mês e no final da pesquisa. 


\section{Desconfortos e riscos esperados;}

Todos os exames desta pesquisa são seguros e bem tolerados. Entretanto, alguns desconfortos podem ocorrer. De maneira geral pode-se esperar:

1) mamografia: os seios serão comprimidos, podendo trazer dor suportável durante o exame;

2) ultra-sonografia e Papanicolau: pode causar algum desconforto, pois serão realizados por via vaginal;

3) medicamento: como todo medicamento, podem ser esperadas algumas reações em algumas das senhoras. Qualquer um dos medicamentos que estarão sendo usados no tratamento poderá provocar tensão nos seios, náuseas, alterações no peso e do desejo sexual, inchaço no corpo e dores de cabeça.

Paramente podem ocorrer reações mais sérias que precisarão ser comunicadas ao seu médico logo que forem observadas. São elas, dores fortes na barriga, dor e inchaço de início abrupto na perna, dor ou sensação de aperto no peito, pele e olhos amarelados, hepatite, coceira no corpo, ataques epiléticos, tonturas e desmaios freqüentes;

4) o teste de esforço na bicicleta ergométrica poderá trazer-Ihe cansaço, mas somente durante o exame. Em algumas pessoas, que sofrem do coração, mas desconhecem o fato, esse exame poderá detectar o problema, mas também pode provocá-lo. Todos esses exames seram sempre acompanhados por médico para sua completa segurança;

5) o treinamento físico para o grupo sorteado será feito com exercícios adequados à condição física de cada uma das senhoras, sem provocar sobrecargas, não apresentando portanto riscos à saúde;

6) no clampeamento, apesar de rara, pode ocorrer uma queda do açúcar no sangue. Isso pode ser rapidamente corrigido com a reposição de açúcar. Para sua maior segurança, esse exame é sempre acompanhado por médico

7) na microneurografia, durante o exame, podem ocorrer alguns sintomas enquanto o médico estará procurando o nervo na sua perna. Esse exame é feito em uma das suas pernas, logo abaixo do joelho. A senhora permanecerá deitada durante todo o exame. Com uma espécie de caneta, o médico fará a localização do seu nervo dando uns choquinhos que farão com que o seu pé mexa-se sozinho. Após localizar o nervo, o médico colocará 2 agulhinhas finas na sua perna. Quando isso ocorrer, a senhora poderá sentir uma leve dor no local, formigamento na perna e no pé, uma sensação de peso na perna, choquinhos ou ainda o pé mexer-se sozinho. Esses sintomas podem ou não aparecer e, se ocorrerem, será somente enquanto o médico estiver procurando o seu nervo. Depois o exame transcorre sem que a senhora sinta qualquer sintoma. Muito raramente, após o exame, pode ocorrer uma certa dormência ou fraqueza na perna onde o exame foi feito. Mesmo quando isso acontece, dura no máximo uma semana, desaparecendo por completo; 
8) durante a entrevista, onde a senhora irá dar a sua opinião sobre o tratamento, poderá haver ansiedade ou mesmo algum constrangimento.

\section{Benefícios que poderão ser obtidos;}

Sem nenhum gasto, as senhoras farão diversas avaliações: clínica, da obesidade, da hipertensão, do colesterol alto, de outra gorduras no sangue, do diabetes, de problemas cardíacos e da condição física, além da menopausa propriamente dita. Todos esses resultados serão informados e, caso seja detectada alguma anormalidade, as senhoras serão encaminhadas para o tratamento dentro do próprio Hospital das Clínicas.

As senhoras receberão, além do tratamento da menopausa, a oportunidade de participar de um programa de treinamento físico adequado às suas condições físicas, que poderá ajudar a prevenir problemas cardíacos.

Nem todos os exames que serão feitos trarão um benefício imediato para a senhora, mas servirão para que os médicos compreendam melhor as alterações que ocorrem no organismo da mulher na menopausa, podendo no futuro melhorar ainda mais o seu tratamento.

\section{Procedimentos alternativos que possam ser vantajosos para o indivíduo.}

Todos os exames realizados e especialmente aqueles das sessões experimentais, podem trazer uma visão detalhada de como está seu organismo. Ao final da pesquisa, se desejado, a senhora poderá requisitar uma prescrição de atividades físicas para seu dia-a-dia.

\section{IV - ESCLARECIMENTOS DADOS PELO PESQUISADOR SOBRE GARANTIAS DO SUJEITO DA PESQUISA CONSIGNANDO:}

1. Acesso, a qualquer tempo, às informações sobre procedimentos, riscos e benefícios relacionados à pesquisa, inclusive para dirimir eventuais dúvidas.

A senhora terá acesso quando quiser às informações constantes nesta declaração ou qualquer outra informação que deseje, sobre seus exames, sobre o medicamento ou sobre este estudo. Esta declaração ficará arquivada em seu prontuário como todas as outras informações, e a senhora pode exigi-las quando quiser. Também pode perguntar o que quiser a qualquer momento que a senhora será esclarecida. 
2. Liberdade de retirar seu consentimento a qualquer momento e de deixar de participar do estudo, sem que isto traga prejuízo à continuidade da assistência.

A senhora não é obrigada a participar deste estudo. A senhora pode se recusar a participar deste estudo e pode também desistir de participar a qualquer momento, sem ter que fornecer razões para sua desistência. Sua recusa não afetará seu direito a um tratamento médico, nem Ihe trará conseqüências negativas.

3. Salvaguarda da confidencialidade, sigilo e privacidade.

Os dados coletados durante este estudo (dados eletrônicos impressos, sua identificação, sua ficha clínica ou prontuário, esta declaração, os resultados de exames, etc.) serão mantidos em sigilo e só serão usados para os fins do tratamento aqui proposto.

Apenas a senhora, os médicos e os membros do Comitê de Ética e autoridades regulatórias terão acesso às informações do estudo, evitando mencionar seu nome ou seus dados pessoais sempre que possível, durante todo o estudo e também na publicação dos resultados.

4. Disponibilidade de assistência no HCFMUSP, por eventuais danos à saúde, decorrentes da pesquisa.

Os médicos envolvidos neste estudo e o Hospital das Clínicas da FMUSP são responsáveis por todo e qualquer problema clínico conseqüente da realização dos exames e do uso da medicação do estudo, e se comprometem a disponibilizar a assistência e não medir esforços na reparação e tratamento de eventuais danos a sua saúde decorrentes deste estudo.

5. Viabilidade de indenização por eventuais danos à saúde decorrentes da pesquisa.

\section{INFORMAÇÕES DE NOMES, ENDEREÇOS E TELEFONES DOS RESPONSÁVEIS PELO ACOMPANHAMENTO DA PESQUISA, PARA CONTATO EM CASO DE INTERCORRÊNCIAS CLÍNICAS E REAÇÕES ADVERSAS.}

Profa. Dra. Angela Maggio da Fonseca

Prof. Dr. Décio Mion Junior

Dra. Taís Tinucci

Dra. Sandra Baliero Abrahão

Dra Claudia Lúcia de Moraes Forjaz

Prof. Crivaldo Gomes Cardoso Júnior 
Prof. Fernanda Rocehi Bernardo

Prof. Bruna Oneda

Prof $^{a}$ Carolina Kimie Moriyama

Enf ${ }^{\mathrm{a}}$ Edna Caetano Ignês

Enf ${ }^{a}$ Josiane Costa de Lima

Dr Fabrício Collares Rosas (Tel: Residência 5031-5160 / Celular 9215-2851)

Av. Dr. Enéas de Carvalho Aguiar, $25510^{\circ}$ andar ICHC - Tel 3069-7223

\section{OBSERVAÇÕES COMPLEMENTARES:}

\section{VII - CONSENTIMENTO PÓS-ESCLARECIDO}

Declaro que, após convenientemente esclarecido pelo pesquisador e ter entendido o que me foi explicado, consinto em participar do presente Protocolo de Pesquisa.

São Paulo, de. de $200 \ldots$

Assinatura do sujeito da pesquisa ou responsável legal
Assinatura do pesquisador (carimbo ou nome legível) 
ANEXO C

Índice Menopausal de Kupperman (IMK)

\begin{tabular}{|l|c|c|c|}
\hline \multicolumn{1}{|c|}{ SINTOMAS } & $\mathbf{L}^{*}$ & $\mathbf{M}^{*}$ & $\mathbf{I}^{*}$ \\
\hline FOGACHOS & 4 & 8 & 12 \\
\hline PARESTESIA & 2 & 4 & 6 \\
\hline INSÔNIA & 2 & 4 & 6 \\
\hline NERVOSISMO & 2 & 4 & 6 \\
\hline MELANCOLIA & 1 & 2 & 3 \\
\hline VERTIGEM & 1 & 2 & 3 \\
\hline FRAQUEZA & 1 & 2 & 3 \\
\hline ARTRALGIA OU MIALGIA & 1 & 2 & 3 \\
\hline CEFALÉIA & 1 & 2 & 3 \\
\hline PALPITAÇÃO & 1 & 2 & 3 \\
\hline FORMIGAMENTO & 1 & 2 & 3 \\
\hline
\end{tabular}

\section{Legenda:}

$\mathbf{L}=$ leve (até 19 pontos)

$\mathbf{M}=$ moderado (de 20 a 35 pontos)

$\mathbf{I}=$ Intenso (acima de 35 pontos) 
ANEXO D - Características da amostra nas sessões PRÉ e PÓS

Características antropométricas

\begin{tabular}{|c|c|c|c|c|c|c|c|c|c|}
\hline \multirow[t]{2}{*}{ Voluntárias } & \multirow[t]{2}{*}{ Grupos } & \multicolumn{2}{|c|}{ Peso } & \multicolumn{2}{|c|}{ Estat. } & \multicolumn{2}{|c|}{ IMC } & \multicolumn{2}{|c|}{ IK } \\
\hline & & PRE & POS & PRE & POS & PRE & POS & PRE & POS \\
\hline MMC & SED-PLA & 56,5 & 52,8 & 1,50 & 1,49 & 25,1 & 23,8 & 31 & 26 \\
\hline MARO & SED-PLA & 67,9 & 71,1 & 1,55 & 1,56 & 28,3 & 29,2 & 12 & 15 \\
\hline RCA & SED-PLA & 67,3 & 74,3 & 1,51 & 1,51 & 29,5 & 32,6 & 34 & 17 \\
\hline MYI & SED-PLA & 62,1 & 64,6 & 1,59 & 1,58 & 24,6 & 25,9 & 19 & 13 \\
\hline MRS & SED-PLA & 46,9 & 46,5 & 1,51 & 1,52 & 20,6 & 20,1 & 31 & 11 \\
\hline FSP & SED-PLA & 51,3 & 51,9 & 1,42 & 1,42 & 25,4 & 25,7 & 4 & 0 \\
\hline BFR & SED-PLA & 58,8 & 59,0 & 1,56 & 1,56 & 24,2 & 24,2 & 12 & 19 \\
\hline VLO & SED-PLA & 57,2 & 54,4 & 1,53 & 1,52 & 24,4 & 23,5 & 8 & 11 \\
\hline LMLM & SED-PLA & 57,3 & 56,8 & 1,55 & 1,55 & 23,9 & 23,6 & 25 & 19 \\
\hline MDB & SED-PLA & 64,3 & 63,5 & 1,63 & 1,63 & 24,2 & 23,9 & 13 & 12 \\
\hline MVL & SED-PLA & 58 & - & 1,61 & - & 22,3 & - & - & - \\
\hline POS & SED-TRH & 59,1 & 58,9 & 1,56 & 1,56 & 24,3 & 24,2 & 20 & 14 \\
\hline EJGV & SED-TRH & 54,7 & 54,5 & 1,61 & 1,61 & 21,1 & 21,0 & 31 & 32 \\
\hline LMS & SED-TRH & 80,7 & 80,4 & 1,66 & 1,65 & 29,3 & 29,5 & 30 & 15 \\
\hline AAR & SED-TRH & 55,5 & 57,7 & 1,54 & 1,55 & 23,4 & 24,0 & 16 & 14 \\
\hline MUNO & SED-TRH & 79,6 & 80,5 & 1,64 & 1,63 & 29,6 & 30,3 & 18 & 8 \\
\hline SRGP & SED-TRH & 70,4 & 70,5 & 1,55 & 1,56 & 29,3 & 29,0 & 25 & 2 \\
\hline JJS & SED-TRH & 59,5 & 53,6 & 1,60 & 1,61 & 23,2 & 20,7 & 34 & 23 \\
\hline NPB & SED-TRH & 83,5 & 83,9 & 1,67 & 1,66 & 29,9 & 30,4 & 13 & 6 \\
\hline RFS & SED-TRH & 69,0 & 71,0 & 1,68 & 1,67 & 24,4 & 25,5 & 15 & 5 \\
\hline SMD & SED-TRH & 55,5 & 55,9 & 1,55 & 1,55 & 23,1 & 23,3 & - & - \\
\hline GG & SED-TRH & 62,3 & 61,5 & 1,62 & 1,62 & 23,7 & 23,4 & 26 & 5 \\
\hline MESS & SED-TRH & 49,3 & 50,4 & 1,47 & 1,47 & 22,8 & 23,3 & 15 & 8 \\
\hline VLCO & SED-TRH & 66,9 & 68,0 & 1,59 & 1,59 & 26,5 & 26,9 & - & - \\
\hline VFS & SED-TRH & 48,05 & 48 & 1,49 & 1,49 & 21,6 & - & - & - \\
\hline SM & TF-PLA & 74,9 & 69,7 & 1,67 & 1,67 & 26,9 & 25,0 & 12 & 43 \\
\hline ECB & TF-PLA & 67,1 & 67,1 & 1,58 & 1,57 & 26,9 & 27,2 & 45 & 31 \\
\hline SRS & TF-PLA & 82,3 & 87,8 & 1,66 & 1,64 & 29,9 & 32,6 & 4 & 3 \\
\hline MCSA & TF-PLA & 62,6 & 61,7 & 1,66 & 1,66 & 22,7 & 22,4 & 38 & 11 \\
\hline AAOS & TF-PLA & 64,8 & 64,2 & 1,59 & 1,58 & 25,6 & 25,7 & 14 & 3 \\
\hline SMMS & TF-PLA & 55,4 & 56,0 & 1,54 & 1,55 & 23,4 & 23,3 & 39 & 17 \\
\hline ITS & TF-PLA & 53,1 & 53,7 & 1,51 & 1,51 & 23,3 & 23,6 & 13 & 4 \\
\hline MACS & TF-PLA & 69,8 & 71,2 & 1,54 & 1,53 & 29,4 & 30,4 & 38 & 12 \\
\hline SCS & TF-PLA & 64,0 & 63,8 & 1,65 & 1,65 & 23,5 & 23,4 & 10 & 3 \\
\hline WGF & TF-PLA & 75,0 & 73,0 & 1,59 & 1,59 & 29,7 & 28,9 & 7 & 1 \\
\hline JBP & TF-PLA & 69,9 & 69,6 & 1,59 & 1,58 & 27,6 & 27,9 & 5 & 4 \\
\hline DMB & TF-PLA & 59,4 & 59,4 & 1,58 & 1,57 & 23,8 & 24,1 & 11 & 7 \\
\hline EACB & TF-TRH & 75,6 & 77,9 & 1,64 & 1,64 & 28,1 & 29,0 & 5 & 11 \\
\hline SMS & TF-TRH & 72,3 & 78,6 & 1,57 & 1,57 & 29,3 & 31,9 & 1 & 0 \\
\hline EMPS & TF-TRH & 57,8 & 56,4 & 1,50 & 1,51 & 25,7 & 24,7 & 15 & 4 \\
\hline ALCLM & TF-TRH & 78,8 & 79,1 & 1,64 & 1,64 & 29,3 & 29,4 & 27 & 9 \\
\hline ORS & TF-TRH & 59,4 & 56,0 & 1,54 & 1,53 & 25,0 & 23,9 & 39 & 35 \\
\hline MAO & TF-TRH & 71,5 & 72,7 & 1,55 & 1,55 & 29,8 & 30,3 & 7 & 0 \\
\hline SSP & TF-TRH & 57,9 & 56,9 & 1,63 & 1,62 & 21,8 & 21,7 & 17 & 0 \\
\hline NVM & TF-TRH & 57,0 & 57,4 & 1,57 & 1,55 & 23,1 & 23,9 & 27 & 6 \\
\hline
\end{tabular}

Peso em Kg; Est=estatura em m; IMC= Índice de massa corporal em kg/m²; $\mathrm{IK}=$ indice menopausal de Kupperman 
Pressão arterial sistólica (PAS), pressão arterial diastólica (PAD) e pressão arterial media (PAM) em $\mathrm{mm} \mathrm{Hg}$

\begin{tabular}{|c|c|c|c|c|c|c|c|}
\hline \multirow[t]{2}{*}{ Voluntárias } & \multirow[t]{2}{*}{ Grupos } & \multicolumn{2}{|c|}{ PAS } & \multicolumn{2}{|c|}{ PAD } & \multicolumn{2}{|c|}{ PAM } \\
\hline & & PRÉ & PÓS & PRÉ & PÓS & PRÉ & PÓS \\
\hline MMC & SED-PLA & 111 & 97 & 71 & 68 & 84 & 78 \\
\hline MARO & SED-PLA & 126 & 120 & 79 & 82 & 95 & 95 \\
\hline RCA & SED-PLA & 114 & 130 & 78 & 82 & 90 & 98 \\
\hline MYI & SED-PLA & 125 & 132 & 85 & 90 & 98 & 104 \\
\hline MRS & SED-PLA & 115 & 118 & 75 & 70 & 88 & 86 \\
\hline FSP & SED-PLA & 131 & 120 & 72 & 68 & 92 & 85 \\
\hline BFR & SED-PLA & 134 & 123 & 83 & 76 & 100 & 92 \\
\hline VLO & SED-PLA & 126 & 118 & 86 & 72 & 99 & 87 \\
\hline LMLM & SED-PLA & 113 & 140 & 70 & 80 & 84 & 100 \\
\hline MDB & SED-PLA & 110 & 107 & 80 & 63 & 90 & 78 \\
\hline MVL & SED-PLA & 130 & - & 70 & - & 90 & - \\
\hline POS & SED-TRH & 123 & 119 & 68 & 81 & 86 & 94 \\
\hline EJGV & SED-TRH & 107 & 106 & 70 & 72 & 82 & 83 \\
\hline LMS & SED-TRH & 107 & 129 & 71 & 74 & 83 & 92 \\
\hline AAR & SED-TRH & 122 & 142 & 77 & 79 & 92 & 100 \\
\hline MUNO & SED-TRH & 125 & 127 & 71 & 78 & 89 & 94 \\
\hline SRGP & SED-TRH & 115 & 111 & 65 & 85 & 82 & 94 \\
\hline JJS & SED-TRH & 127 & 135 & 64 & 64 & 85 & 88 \\
\hline NPB & SED-TRH & 121 & 105 & 75 & 63 & 90 & 77 \\
\hline RFS & SED-TRH & 110 & 97 & 76 & 73 & 87 & 81 \\
\hline SMD & SED-TRH & 126 & 112 & 65 & 66 & 85 & 81 \\
\hline GG & SED-TRH & 114 & 110 & 68 & 72 & 83 & 85 \\
\hline MESS & SED-TRH & 138 & 145 & 86 & 85 & 103 & 105 \\
\hline VLCO & SED-TRH & 100 & 105 & 65 & 67 & 77 & 80 \\
\hline VFS & SED-TRH & 123 & 111 & 77 & 68 & 92 & 84 \\
\hline SM & TF-PLA & 102 & 124 & 68 & 80 & 79 & 95 \\
\hline ECB & TF-PLA & 131 & 125 & 79 & 75 & 96 & 92 \\
\hline SRS & TF-PLA & 122 & 112 & 84 & 77 & 97 & 89 \\
\hline MCSA & TF-PLA & 117 & 124 & 81 & 73 & 93 & 90 \\
\hline AAOS & TF-PLA & 130 & 113 & 80 & 68 & 97 & 83 \\
\hline SMMS & TF-PLA & 112 & 109 & 78 & 76 & 89 & 87 \\
\hline ITS & TF-PLA & 121 & 119 & 75 & 74 & 90 & 89 \\
\hline MACS & TF-PLA & 119 & 129 & 80 & 90 & 93 & 103 \\
\hline SCS & TF-PLA & 115 & 97 & 77 & 74 & 90 & 82 \\
\hline WGF & TF-PLA & 119 & 121 & 87 & 83 & 98 & 96 \\
\hline JBP & TF-PLA & 124 & 120 & 82 & 70 & 96 & 87 \\
\hline DMB & TF-PLA & 104 & 116 & 72 & 68 & 83 & 84 \\
\hline EACB & TF-TRH & 120 & 115 & 76 & 68 & 91 & 84 \\
\hline SMS & TF-TRH & 130 & 135 & 86 & 85 & 101 & 102 \\
\hline EMPS & TF-TRH & 135 & 122 & 86 & 71 & 102 & 88 \\
\hline ALCLM & TF-TRH & 138 & 148 & 85 & 85 & 103 & 106 \\
\hline ORS & TF-TRH & 133 & 104 & 71 & 70 & 92 & 81 \\
\hline MAO & TF-TRH & 100 & 102 & 74 & 71 & 83 & 81 \\
\hline SSP & TF-TRH & 106 & 118 & 63 & 71 & 77 & 87 \\
\hline NVM & TF-TRH & 116 & 121 & 72 & 78 & 87 & 94 \\
\hline
\end{tabular}


Taxas hormonais

\begin{tabular}{|c|c|c|c|c|c|c|c|}
\hline Voluntárias & Grupos & $\mathbf{E}$ & $E$ & LH & LH & FSH & FSH \\
\hline & & PRÉ & PÓS & PRÉ & PÓS & PRÉ & PÓS \\
\hline MMC & SED-PLA & 13 & 13 & 39,1 & 35,8 & 108 & 112,3 \\
\hline MARO & SED-PLA & 30,5 & 13 & 13,9 & 16,1 & 51 & 61,1 \\
\hline RCA & SED-PLA & 13 & 13 & 22,1 & 25,7 & 57,8 & 57,2 \\
\hline MYI & SED-PLA & 13 & 13 & 41,3 & 29,8 & 65,3 & 65,7 \\
\hline MRS & SED-PLA & 12,9 & 45,6 & 15,2 & 39,5 & 46,2 & 74,9 \\
\hline FSP & SED-PLA & 13 & 18,5 & 63,9 & 60,1 & 131 & 138,7 \\
\hline BFR & SED-PLA & 13 & 13 & 55,6 & 38,8 & 103,4 & 97,3 \\
\hline VLO & SED-PLA & 13 & 13 & 42,2 & 55,1 & 109,3 & 115 \\
\hline LMLM & SED-PLA & 13 & 13 & 37,6 & 39,9 & 94,3 & 82 \\
\hline MDB & SED-PLA & 13 & 13 & 22,9 & 33,8 & 61,1 & 74,4 \\
\hline MVL & SED-PLA & 35 & - & 47 & - & 111 & \\
\hline POS & SED-TRH & 13 & 49,5 & 35,4 & 30,1 & 41,2 & 40,3 \\
\hline EJGV & SED-TRH & 18,4 & 42,4 & 19,6 & 18,7 & 51 & 47,6 \\
\hline LMS & SED-TRH & 13 & 17,8 & 64,5 & 62,6 & 125 & 126,2 \\
\hline AAR & SED-TRH & 13,8 & 94,6 & 25 & 10,2 & 63,2 & 21,5 \\
\hline MUNO & SED-TRH & 13 & 53,5 & 30,4 & 26,9 & 64,3 & 42.2 \\
\hline SRGP & SED-TRH & 13 & 29,3 & 38,9 & 43 & 77,4 & 52 \\
\hline JJS & SED-TRH & 13,9 & 115,4 & 39,6 & 53 & 41,1 & 91 \\
\hline NPB & SED-TRH & 13 & 31,8 & 22,1 & 13,9 & 52,9 & 40,3 \\
\hline RFS & SED-TRH & 13 & 21 & 71,1 & 58,9 & 138,3 & 90,1 \\
\hline SMD & SED-TRH & 13 & 39 & 63,9 & 52,5 & 144,9 & 130,2 \\
\hline GG & SED-TRH & 13 & 44 & 28,7 & 31,8 & 78 & 66,3 \\
\hline MESS & SED-TRH & 13 & 56 & 53 & 35,2 & 97,8 & 37,9 \\
\hline VLCO & SED-TRH & 17,9 & 15 & 55,3 & 49,3 & 82,3 & 83,4 \\
\hline VFS & SED-TRH & 13 & 57 & 44,7 & 30,8 & 113,1 & 54 \\
\hline SM & TF-PLA & 13 & 13 & 41,9 & 50,4 & 98,8 & 133,6 \\
\hline ECB & TF-PLA & 13 & 19,1 & 22,6 & 19,8 & 64,5 & 58,7 \\
\hline SRS & TF-PLA & 13 & 32,1 & 10 & 13,1 & 38,7 & 11,7 \\
\hline MCSA & TF-PLA & 14,9 & 13 & 58,6 & 36,6 & 99,5 & 103,6 \\
\hline AAOS & TF-PLA & 13 & 16,7 & 29,5 & 36,1 & 70,1 & 69,8 \\
\hline SMMS & TF-PLA & 13 & 13 & 67,1 & 68,1 & 151,4 & 163,2 \\
\hline ITS & TF-PLA & 13 & 13 & 22,5 & 33,5 & 86,5 & 95,1 \\
\hline MACS & TF-PLA & 13 & 13,6 & 34,8 & 27,3 & 62,8 & 55,3 \\
\hline SCS & TF-PLA & 24 & 18 & 34,5 & 26 & 63,7 & 52,7 \\
\hline WGF & TF-PLA & 13,5 & 19 & 25,2 & 22,5 & 62,1 & 52,3 \\
\hline JBP & TF-PLA & 14,3 & 13 & 43,8 & 31,9 & 83,4 & 69 \\
\hline DMB & TF-PLA & 93 & 39 & 37,7 & 22,1 & 40,3 & 28,9 \\
\hline EACB & TF-TRH & 13 & 45,9 & 19 & 15,8 & 52 & 39,3 \\
\hline SMS & TF-TRH & 13 & 43,7 & 39,7 & 25,3 & 113 & 55,8 \\
\hline EMPS & TF-TRH & 13 & 109,7 & 38,2 & 23 & 73,8 & 48,2 \\
\hline ALCLM & TF-TRH & 14,3 & 36 & 44,8 & 50,9 & 92 & 72 \\
\hline ORS & TF-TRH & 19,3 & 13 & 27,8 & 19,4 & 79,2 & 55,7 \\
\hline MAO & TF-TRH & 14,5 & 69,5 & 53,1 & 57,4 & 85,7 & 59,7 \\
\hline SSP & TF-TRH & 15 & 13 & 44,3 & 48,8 & 111 & 98,6 \\
\hline NVM & TF-TRH & 47 & 53 & 27,2 & 52,8 & 43,7 & 79,2 \\
\hline
\end{tabular}

$\mathrm{E}=$ estradiol em $\mathrm{pg} / \mathrm{ml}$; $\mathrm{LH}=$ hormônio luteinizante em $\mu \mathrm{m} / \mathrm{ml} ; \mathrm{FSH}=$ hormônio folículo estimulante em $\mu \mathrm{m} / \mathrm{ml}$ 
Dados dos testes ergoespirométricos

\begin{tabular}{|c|c|c|c|c|c|c|c|}
\hline Voluntárias & Grupos & LA & LA & PCR & PCR & $\mathrm{VO}_{2 \text { máx }}$ & $\mathrm{VO}_{2 \text { máx }}$ \\
\hline & & PRÉ & PÓS & PRÉ & PÓS & PRÉ & PÓS \\
\hline MMC & SED-PLA & 10,7 & 9,3 & 16,4 & 14,1 & 22,6 & 19,7 \\
\hline MARO & SED-PLA & 8,4 & 8,8 & 12,7 & 14,7 & 16,9 & 15,7 \\
\hline RCA & SED-PLA & 11,2 & 13 & 17 & 17,3 & 19,6 & 19 \\
\hline MYI & SED-PLA & 9,2 & 4,8 & 13,3 & 14 & 14,5 & 17,7 \\
\hline MRS & SED-PLA & 9,1 & 4,4 & 10,6 & 10,3 & 10,3 & 15,6 \\
\hline FSP & SED-PLA & 12,7 & 11,9 & 14,8 & 20,2 & 16,7 & 21,8 \\
\hline BFR & SED-PLA & 12,4 & 12,9 & 18,2 & 15,8 & 23,5 & 22,9 \\
\hline LMLM & SED-PLA & 10,7 & 5,6 & 18,1 & 15,3 & 19,3 & 18,2 \\
\hline MDB & SED-PLA & 8,1 & 6,2 & 16,2 & 14,8 & 19,8 & - \\
\hline POS & SED-TRH & 6,3 & 6,4 & 12,5 & 12,1 & 13,5 & 14,6 \\
\hline EJGV & SED-TRH & 10,9 & 11,3 & 16,6 & 21,1 & 20,6 & 22,4 \\
\hline LMS & SED-TRH & 5,0 & 5 & 11 & 11 & 15,4 & 15,7 \\
\hline AAR & SED-TRH & 8,8 & 9,6 & 13,1 & 15,8 & 14,4 & 20,7 \\
\hline MUNO & SED-TRH & 8,8 & 9,9 & & & 12,3 & 13,3 \\
\hline SRGP & SED-TRH & 9,1 & 8,3 & 13,1 & 13,4 & 17,9 & 16,2 \\
\hline JJS & SED-TRH & 10,8 & 13,9 & 20,9 & 15,9 & 23,2 & 20,3 \\
\hline NPB & SED-TRH & 11 & 11 & 16 & 19,2 & 18,1 & 22,5 \\
\hline RFS & SED-TRH & 10,6 & 5,6 & 15,6 & 11,7 & 16,9 & 17,8 \\
\hline SMD & SED-TRH & 13,3 & 8,8 & 21,1 & 20 & 21,9 & 20,8 \\
\hline GG & SED-TRH & 7,4 & 8,7 & 16,6 & 15,7 & 18,7 & 19,3 \\
\hline MESS & SED-TRH & 13,3 & 15 & 17,3 & 18,1 & 20,7 & 22,7 \\
\hline VLCO & SED-TRH & 10,0 & 8,20 & 16,0 & 19,2 & - & - \\
\hline VFG & SED-TRH & 13,4 & 9 & 16,2 & 13,5 & 19,5 & 15,6 \\
\hline ECB & TF-PLA & 13,3 & 13,5 & 16,2 & 17,9 & 19 & 20,5 \\
\hline SRS & TF-PLA & 12,5 & 5,9 & 17,2 & 16,4 & 20,4 & 21 \\
\hline MCSA & TF-PLA & 10,9 & 11,7 & 18,7 & - & 20,2 & 22 \\
\hline AAOS & TF-PLA & 8,2 & 7,9 & 15,1 & 19 & 17,3 & 20,2 \\
\hline SMMS & TF-PLA & 10,0 & 17,3 & 16,1 & 22,5 & 17,8 & 28,8 \\
\hline ITS & TF-PLA & 9,0 & 13,3 & 18,6 & 22,1 & 19,3 & 29 \\
\hline MACS & TF-PLA & 10,5 & 16,8 & 14,8 & 26,4 & 18,9 & 31 \\
\hline SCS & TF-PLA & 10,3 & 11,4 & 14,8 & 19,8 & 23,9 & 23,8 \\
\hline WGF & TF-PLA & 12,9 & 16,1 & 23,3 & 26,5 & 25 & 31 \\
\hline JBP & TF-PLA & 10,6 & 15 & 15,6 & 23,9 & 17,8 & 28,3 \\
\hline DMB & TF-PLA & 11,6 & 16,3 & 23,6 & 27,4 & 25,6 & 32,3 \\
\hline EACB & TF-TRH & 6,8 & 10,5 & 12,1 & 18,6 & 16,4 & 20,4 \\
\hline SMS & TF-TRH & 7,5 & 8,1 & 14,4 & 15,6 & 15,3 & 15,6 \\
\hline EMPS & TF-TRH & 9,3 & 10,4 & 12,4 & 13,9 & 13,2 & 13,9 \\
\hline ALCLM & TF-TRH & 11,1 & 13,5 & 17,3 & 23,2 & 21,3 & 24,6 \\
\hline ORS & TF-TRH & 9,1 & 10 & 11,7 & 14,6 & 16,3 & 22,9 \\
\hline MAO & TF-TRH & 12,4 & 13,9 & 16,5 & 18,5 & 19,9 & 24,5 \\
\hline SSP & TF-TRH & 15 & 18,8 & 17,5 & 29,4 & 18,3 & 31,9 \\
\hline NVM & TF-TRH & 16,3 & 19,7 & 22,2 & 24,6 & 26,4 & 28,2 \\
\hline
\end{tabular}

$\mathrm{LA}=$ limiar anaeróbio em $\mathrm{ml} \cdot \mathrm{kg}^{-1} \mathrm{~min}^{-1}$; $\quad \mathrm{PCR}=$ pico de compesação respiratória em $\mathrm{ml} \mathrm{kg}^{-1} \mathrm{~min}^{-1}$; VO2máx= consumo máximo de oxigênio em $\mathrm{ml} . \mathrm{kg}^{-1} \mathrm{~min}^{-1}$ 


\section{ANEXO E - Dados do período basal}

Valores de ANSP (impulsos/min)

\begin{tabular}{|c|c|c|c|c|c|c|c|c|c|c|c|c|c|}
\hline \multirow[b]{3}{*}{ voluntárias } & \multirow[b]{3}{*}{ Grupo } & \multicolumn{6}{|c|}{ SESSÃO PRÉ } & \multicolumn{6}{|c|}{ SESSÃO PÓS } \\
\hline & & \multicolumn{5}{|c|}{ minutos } & \multirow[b]{2}{*}{ média } & \multicolumn{5}{|c|}{ minutos } & \multirow[b]{2}{*}{ média } \\
\hline & & 1 & 2 & 3 & 4 & 5 & & 1 & 2 & 3 & 4 & 5 & \\
\hline MMC & SED-PLA & - & 62 & 57 & 56 & 39 & 54 & 56 & 41 & 65 & 56 & 47 & 53 \\
\hline MARO & SED-PLA & 27 & 26 & 28 & 25 & 31 & 27 & - & 25 & 29 & 33 & - & 29 \\
\hline RCA & SED-PLA & 57 & 47 & 57 & 54 & 51 & 53 & 55 & 50 & - & - & 40 & 48 \\
\hline MYI & SED-PLA & 38 & 35 & 35 & 38 & - & 37 & 58 & 56 & 58 & 54 & 65 & 58 \\
\hline FSP & SED-PLA & 33 & 34 & 36 & 32 & 34 & 34 & 32 & 35 & 36 & 35 & 31 & 34 \\
\hline VLO & SED-PLA & 43 & 38 & 39 & - & 42 & 41 & 43 & 39 & - & 37 & 43 & 41 \\
\hline BFR & SED-PLA & 23 & 26 & 23 & 23 & 25 & 24 & - & - & - & - & - & - \\
\hline MRS & SED-PLA & 38 & 32 & 38 & 42 & 45 & 39 & 39 & 31 & 34 & 38 & 31 & 35 \\
\hline LMLM & SED-PLA & 37 & 40 & 44 & - & 46 & 42 & - & - & - & - & - & - \\
\hline MDTBS & SED-PLA & - & 42 & 40 & 46 & 41 & 42 & 44 & 39 & 41 & 35 & 38 & 39 \\
\hline MVL & SED-PLA & 40 & 35 & 37 & 33 & - & 36 & 37 & 37 & 35 & 35 & - & 36 \\
\hline POS & SED-TRH & 48 & 39 & 41 & 39 & 48 & 43 & 49 & 42 & 45 & 47 & 44 & 45 \\
\hline EJGV & SED-TRH & 57 & 51 & 48 & - & - & 52 & 44 & 45 & 50 & 40 & 36 & 43 \\
\hline LMS & SED-TRH & 33 & 33 & 32 & 30 & 28 & 31 & 35 & 38 & 35 & 37 & 33 & 36 \\
\hline MUN & SED-TRH & 33 & 40 & 34 & 39 & 33 & 36 & 40 & 39 & 37 & 39 & 37 & 38 \\
\hline JJS & SED-TRH & 40 & 40 & 38 & - & - & 39 & 34 & 31 & 28 & 26 & 28 & 29 \\
\hline NPB & SED-TRH & 36 & 41 & 36 & 33 & 34 & 36 & 30 & 32 & 37 & 39 & 30 & 34 \\
\hline RFS & SED-TRH & 39 & 40 & 40 & 39 & 37 & 39 & 38 & 37 & 39 & 36 & 31 & 36 \\
\hline AAR & SED-TRH & 52 & - & 43 & 47 & 49 & 48 & 39 & 39 & 34 & 38 & 34 & 37 \\
\hline SMDR & SED-TRH & 39 & 37 & 40 & 36 & 34 & 37 & 42 & 41 & 34 & 35 & 36 & 38 \\
\hline MESS & SED-TRH & 41 & 37 & 36 & 35 & 36 & 37 & 40 & 39 & 38 & 40 & - & 39 \\
\hline SRGP & SED-TRH & 32 & 30 & 36 & - & 44 & 36 & 50 & 43 & 42 & 40 & 44 & 44 \\
\hline VLCO & SED-TRH & 41 & 40 & 38 & 42 & 40 & - & - & - & - & - & - & - \\
\hline SM & TF-PLA & - & 28 & 37 & 34 & 35 & 34 & 30 & 31 & 29 & - & - & 30 \\
\hline ECB & TF-PLA & 51 & 48 & 48 & - & - & 49 & 36 & 34 & 27 & 35 & - & 33 \\
\hline SRS & TF-PLA & - & - & - & - & - & 37 & 34 & 28 & 31 & 36 & 30 & 32 \\
\hline MCSA & TF-PLA & 40 & 40 & 48 & 37 & - & 41 & 35 & - & 30 & 28 & 30 & 31 \\
\hline AAOS & TF-PLA & - & - & 27 & 28 & 30 & 28 & - & - & - & - & - & 26 \\
\hline ITS & TF-PLA & 37 & 30 & 30 & - & 37 & 35 & 36 & - & 30 & 42 & - & 36 \\
\hline MACS & TF-PLA & - & - & - & - & - & 40 & 41 & 35 & 39 & 31 & 39 & 37 \\
\hline SCS & TF-PLA & 30 & 33 & 39 & 36 & 36 & 35 & 37 & 36 & 35 & 27 & 29 & 33 \\
\hline WGF & TF-PLA & 44 & 38 & 42 & 38 & 39 & 40 & 40 & 40 & 41 & 32 & 36 & 38 \\
\hline JMBP & TF-PLA & 39 & - & 39 & 48 & 47 & 43 & 43 & 45 & 44 & 40 & 42 & 43 \\
\hline DMB & TF-PLA & 42 & 41 & 33 & 37 & 43 & 39 & 33 & 30 & 33 & - & - & 32 \\
\hline EMCB & TF-TRH & - & - & - & - & - & 40 & 36 & 39 & 36 & 38 & 31 & 36 \\
\hline SMS & TF-TRH & 50 & 46 & 55 & - & - & 50 & & 55 & 45 & 48 & 37 & 46 \\
\hline ALCLM & TF-TRH & 47 & 50 & 43 & 48 & 50 & 48 & 44 & 35 & 38 & - & - & 39 \\
\hline ORS & TF-TRH & & & 36 & 32 & 33 & 34 & 33 & 30 & 35 & 36 & 32 & 33 \\
\hline MAO & TF-TRH & 26 & 29 & 29 & 28 & 38 & 30 & 32 & 31 & 31 & 32 & 31 & 31 \\
\hline EMPS & TF-TRH & 39 & 38 & 38 & 38 & - & 38 & 30 & 33 & 28 & 30 & 35 & 31 \\
\hline
\end{tabular}


Valores de PAS ( $\mathrm{mm} \mathrm{Hg}$ )

\begin{tabular}{|c|c|c|c|c|c|c|c|c|c|c|c|c|c|}
\hline \multirow[b]{3}{*}{ voluntárias } & \multirow[b]{3}{*}{ grupo } & \multicolumn{6}{|c|}{ SESSÃO PRÉ } & \multicolumn{6}{|c|}{ SESSÃO PÓS } \\
\hline & & \multicolumn{5}{|c|}{ minutos } & \multirow[b]{2}{*}{ média } & \multicolumn{5}{|c|}{ minutos } & \multirow[b]{2}{*}{ média } \\
\hline & & 1 & 2 & 3 & 4 & 5 & & 1 & 2 & 3 & 4 & 5 & \\
\hline MMC & SED-PLA & & & 127 & 134 & 134 & 132 & 132 & 127 & 128 & 128 & & 129 \\
\hline MARO & SED-PLA & 173 & 178 & 174 & 173 & 163 & 172 & 176 & 180 & 164 & 163 & 163 & 169 \\
\hline RCA & SED-PLA & 150 & 151 & 163 & 164 & 158 & 157 & 175 & 177 & 178 & 164 & 177 & 174 \\
\hline MYI & SED-PLA & 157 & 151 & 144 & 144 & 158 & 151 & 149 & 149 & 156 & 143 & 149 & 149 \\
\hline FSP & SED-PLA & 143 & 128 & 124 & 129 & 134 & 132 & 150 & 163 & 145 & 149 & 155 & 152 \\
\hline VLO & SED-PLA & 165 & 166 & 157 & 159 & 159 & 161 & 150 & 154 & 152 & 150 & 149 & 151 \\
\hline BFR & SED-PLA & 150 & 156 & 143 & 144 & 155 & 150 & 142 & 138 & 135 & 141 & 141 & 139 \\
\hline MRS & SED-PLA & 142 & 143 & 142 & 163 & 149 & 148 & 127 & 135 & 134 & 136 & 134 & 133 \\
\hline LMLM & SED-PLA & 147 & 146 & 142 & 141 & 136 & 142 & 149 & 149 & 142 & 136 & 142 & 144 \\
\hline MDTBS & SED-PLA & 141 & 135 & 133 & 129 & 134 & 134 & 137 & 133 & 133 & 120 & 127 & 130 \\
\hline$\overline{M V L}$ & SED-PLA & 136 & 130 & 132 & 133 & 146 & 135 & 154 & 147 & 145 & 147 & 149 & 148 \\
\hline POS & SED-TRH & - & - & 146 & 153 & 160 & 153 & 145 & 152 & 147 & 146 & - & 148 \\
\hline EJGV & SED-TRH & 153 & 160 & - & - & - & 157 & 134 & 149 & 148 & 141 & 143 & 143 \\
\hline LMS & SED-TRH & - & 168 & 156 & 162 & - & 162 & 159 & 160 & 175 & 169 & 169 & 166 \\
\hline MUN & SED-TRH & 143 & 152 & 134 & 149 & 142 & 144 & 176 & 162 & 147 & 156 & 149 & 158 \\
\hline JJS & SED-TRH & 172 & 165 & 171 & 172 & - & 170 & 142 & 148 & 144 & 143 & 150 & 145 \\
\hline NPB & SED-TRH & 157 & 162 & 171 & 164 & 170 & 165 & 184 & 170 & 163 & 164 & 163 & 169 \\
\hline RFS & SED-TRH & 115 & 103 & 111 & 91 & 119 & 108 & 133 & 119 & 119 & 132 & 119 & 124 \\
\hline AAR & SED-TRH & 166 & 164 & 161 & 154 & 142 & 157 & 166 & 167 & 158 & 158 & 151 & 160 \\
\hline SMDR & SED-TRH & - & 149 & 146 & 124 & - & 140 & 136 & 159 & 149 & 140 & 142 & 145 \\
\hline GG & SED-TRH & 144 & 140 & 135 & 147 & 142 & 142 & 144 & 146 & 146 & 139 & 142 & 143 \\
\hline MESS & SED-TRH & 162 & 162 & 164 & 155 & 162 & 161 & 155 & 160 & 154 & 157 & 157 & 157 \\
\hline SRGP & SED-TRH & 136 & 143 & 144 & 137 & 149 & 142 & 124 & 137 & 144 & 136 & 137 & 136 \\
\hline VFG & SED-TRH & 138 & 119 & 135 & 140 & 141 & 135 & 143 & 136 & 132 & 140 & 133 & 137 \\
\hline ECB & TF-PLA & 161 & 149 & 156 & - & - & 155 & 163 & 154 & - & 163 & 168 & 162 \\
\hline SRS & TF-PLA & 161 & 161 & - & - & - & 161 & 134 & 123 & 134 & 135 & 135 & 132 \\
\hline MCSA & TF-PLA & 167 & 167 & 162 & - & - & 165 & 160 & 155 & 148 & 149 & 156 & 154 \\
\hline AAOS & TF-PLA & - & 161 & 161 & 177 & 156 & 164 & 152 & 157 & 156 & 156 & 151 & 154 \\
\hline ITS & TF-PLA & 163 & - & 160 & 162 & 164 & 162 & 137 & 145 & 139 & 132 & 146 & 140 \\
\hline MACS & TF-PLA & - & - & - & - & - & - & 159 & 162 & 155 & 154 & 161 & \\
\hline SCS & TF-PLA & 132 & - & 142 & 141 & 135 & 138 & 133 & 126 & 135 & 128 & 121 & 129 \\
\hline WGF & TF-PLA & 167 & 170 & 175 & 178 & 179 & - & 151 & 147 & 153 & 153 & 153 & \\
\hline JMBP & TF-PLA & 152 & 152 & 154 & 162 & 149 & 154 & 153 & 155 & - & 155 & 157 & 155 \\
\hline DMB & TF-PLA & 124 & 126 & 128 & 127 & 125 & 126 & 124 & 127 & 128 & 136 & 116 & 126 \\
\hline EMCB & TF-TRH & - & - & - & - & - & - & 169 & 163 & 169 & 171 & 185 & - \\
\hline SMS & TF-TRH & 163 & 162 & 155 & - & - & 160 & 161 & - & 163 & 164 & 156 & 161 \\
\hline ALCLM & TF-TRH & 194 & 196 & 192 & 195 & 197 & & 185 & 192 & 187 & - & - & \\
\hline ORS & TF-TRH & 177 & 178 & 172 & 180 & 180 & 177 & 164 & 172 & 157 & 157 & 162 & 162 \\
\hline MAO & TF-TRH & 131 & 130 & 130 & 126 & 138 & 131 & 115 & 107 & 114 & 114 & 96 & 109 \\
\hline SSP & TF-TRH & 126 & 136 & 132 & & 135 & 132 & 148 & - & 148 & 150 & 151 & 149 \\
\hline EMPS & TF-TRH & 153 & 140 & 149 & 155 & 148 & 149 & 141 & 141 & 146 & 140 & 155 & 145 \\
\hline NVM & TF-TRH & 147 & 161 & 152 & 141 & 147 & 150 & 148 & 141 & 142 & 156 & 150 & 147 \\
\hline
\end{tabular}


Valores de PAD ( $\mathrm{mm} \mathrm{Hg})$

\begin{tabular}{|c|c|c|c|c|c|c|c|c|c|c|c|c|c|}
\hline \multirow[b]{3}{*}{ voluntárias } & \multirow[b]{3}{*}{ grupo } & \multicolumn{6}{|c|}{ SESSÃO PRÉ } & \multicolumn{6}{|c|}{ SESSÃO PÓS } \\
\hline & & \multicolumn{5}{|c|}{ minutos } & \multirow[b]{2}{*}{ média } & \multicolumn{5}{|c|}{ minutos } & \multirow[b]{2}{*}{ média } \\
\hline & & 1 & 2 & 3 & 4 & 5 & & 1 & 2 & 3 & 4 & 5 & \\
\hline MMC & SED-PLA & - & - & 59 & 62 & 61 & 61 & 66 & 66 & 67 & 67 & - & 67 \\
\hline MARO & SED-PLA & 92 & 74 & 82 & 78 & 78 & 81 & 82 & 79 & 80 & 79 & 77 & 79 \\
\hline RCA & SED-PLA & 82 & 82 & 81 & 82 & 79 & 81 & 89 & 84 & 86 & 84 & 85 & 86 \\
\hline MYI & SED-PLA & 83 & 86 & 85 & 80 & 90 & 85 & 76 & 77 & 79 & 75 & 85 & 78 \\
\hline FSP & SED-PLA & 68 & - & 61 & 72 & - & 67 & 74 & 72 & 70 & 71 & 74 & 72 \\
\hline VLO & SED-PLA & 79 & 83 & 86 & 87 & 85 & 84 & 83 & 88 & 84 & 80 & 85 & 84 \\
\hline BFR & SED-PLA & 66 & 78 & 66 & 68 & 67 & 69 & 61 & 67 & 68 & 71 & - & 67 \\
\hline MRS & SED-PLA & 78 & 75 & 74 & - & 82 & 77 & 70 & 72 & 71 & 71 & - & 71 \\
\hline LMLM & SED-PLA & 72 & - & 70 & 73 & 72 & 72 & 63 & 61 & 67 & 62 & 63 & 63 \\
\hline MDTBS & SED-PLA & 64 & 66 & 62 & 65 & 58 & 63 & 65 & 61 & 69 & 64 & - & 65 \\
\hline MVL & SED-PLA & - & 68 & 71 & 70 & 75 & 71 & 89 & 71 & 67 & 70 & 63 & 72 \\
\hline POS & SED-TRH & - & - & - & - & - & - & 71 & 93 & 65 & 69 & - & 74 \\
\hline EJGV & SED-TRH & 79 & 72 & - & - & - & 76 & 77 & 69 & 72 & 67 & 69 & 71 \\
\hline LMS & SED-TRH & 77 & 84 & 83 & - & - & 81 & 84 & 73 & 72 & 78 & 78 & 77 \\
\hline MUN & SED-TRH & 69 & 73 & 72 & 68 & 72 & 71 & 73 & 74 & 74 & 72 & 78 & 74 \\
\hline JJS & SED-TRH & 84 & 74 & 81 & 109 & - & 87 & 70 & 67 & - & 65 & 65 & 67 \\
\hline NPB & SED-TRH & 78 & 77 & 93 & 95 & 76 & 84 & 75 & 78 & 81 & 74 & 109 & 83 \\
\hline RFS & SED-TRH & 59 & 54 & 57 & 59 & 58 & 57 & 69 & 67 & 66 & 66 & 62 & 66 \\
\hline AAR & SED-TRH & 78 & 77 & 75 & 74 & 73 & 75 & 83 & 79 & 80 & 83 & 79 & 81 \\
\hline SMDR & SED-TRH & - & 71 & 63 & 59 & - & 64 & 76 & 77 & 71 & 61 & 69 & 71 \\
\hline GG & SED-TRH & 65 & 62 & 66 & 63 & 61 & 63 & 67 & 61 & 62 & 64 & 65 & 64 \\
\hline MESS & SED-TRH & 84 & 82 & 91 & 90 & 91 & 88 & 87 & 91 & 83 & 84 & - & 86 \\
\hline SRGP & SED-TRH & 69 & 62 & 63 & 67 & 61 & 64 & 71 & 75 & 65 & 68 & 67 & 69 \\
\hline VLCO & SED-TRH & 71 & 78 & 74 & 73 & 73 & 74 & - & - & - & - & - & - \\
\hline VFG & SED-TRH & 79 & 68 & 70 & 67 & 67 & 70 & 69 & 71 & 72 & - & 66 & 70 \\
\hline ECB & TF-PLA & 82 & 80 & 77 & - & - & 80 & 80 & 81 & 75 & 72 & 77 & 77 \\
\hline SRS & TF-PLA & 96 & 91 & - & - & - & 94 & 78 & 80 & 81 & 77 & 84 & 80 \\
\hline MCSA & TF-PLA & - & 98 & 96 & - & - & 97 & 88 & 93 & - & 89 & 89 & 90 \\
\hline AAOS & TF-PLA & 81 & 77 & 81 & 82 & 79 & 80 & 80 & 81 & 83 & 78 & 80 & 80 \\
\hline ITS & TF-PLA & 68 & 74 & 75 & 80 & 83 & 76 & 64 & 65 & 70 & 69 & 68 & 67 \\
\hline SCS & TF-PLA & 76 & 70 & 78 & 79 & 65 & 74 & 75 & 77 & - & 72 & 70 & 74 \\
\hline WGF & TF-PLA & 92 & 91 & 90 & 88 & 92 & 91 & 85 & 83 & 79 & 86 & 83 & 83 \\
\hline JMBP & TF-PLA & 83 & 75 & 77 & 75 & 77 & 77 & - & 75 & 83 & 85 & 78 & 80 \\
\hline DMB & TF-PLA & 65 & 58 & 59 & 64 & 58 & 61 & - & 64 & 64 & 63 & 63 & 64 \\
\hline EMCB & TF-TRH & - & - & - & - & - & 90 & 84 & 82 & 81 & 88 & 85 & 84 \\
\hline SMS & TF-TRH & 89 & 88 & 81 & - & - & 86 & 86 & & 82 & 85 & 80 & 83 \\
\hline ALCLM & TF-TRH & - & 108 & 108 & 108 & - & 108 & 88 & 90 & 82 & - & - & 87 \\
\hline ORS & TF-TRH & 83 & 99 & 86 & 82 & 83 & 87 & 76 & 78 & 73 & 73 & 72 & 74 \\
\hline MAO & TF-TRH & 74 & 79 & 75 & 73 & 76 & 75 & 52 & 64 & 58 & 56 & 56 & 57 \\
\hline SSP & TF-TRH & - & 72 & 64 & - & 52 & 63 & 66 & 68 & 66 & - & 65 & 66 \\
\hline EMPS & TF-TRH & 84 & 74 & 72 & 73 & 71 & 75 & 69 & 69 & 71 & 70 & 68 & 69 \\
\hline NVM & TF-TRH & - & 72 & 69 & 70 & 75 & 72 & 74 & - & - & 73 & 75 & 74 \\
\hline
\end{tabular}


Valores de PAM (mm Hg)

\begin{tabular}{|c|c|c|c|c|c|c|c|c|c|c|c|c|c|}
\hline \multirow[b]{3}{*}{ voluntárias } & \multirow[b]{3}{*}{ grupo } & \multicolumn{6}{|c|}{ SESSÃO PRÉ } & \multicolumn{6}{|c|}{ SESSÃO PÓS } \\
\hline & & \multicolumn{5}{|c|}{ minutos } & \multirow[b]{2}{*}{ média } & \multicolumn{5}{|c|}{ minutos } & \multirow[b]{2}{*}{ média } \\
\hline & & 1 & 2 & 3 & 4 & 5 & & 1 & 2 & 3 & 4 & 5 & \\
\hline MMC & SED-PLA & - & - & 79 & 72 & 81 & 77 & 75 & & 72 & 78 & - & 75 \\
\hline MARO & SED-PLA & 125 & 90 & 93 & 91 & 96 & 99 & 100 & 97 & 101 & 94 & 101 & 99 \\
\hline RCA & SED-PLA & 103 & 101 & 91 & 104 & 96 & 99 & 113 & 109 & 101 & 103 & 100 & 105 \\
\hline MYI & SED-PLA & 97 & 100 & 100 & 94 & 104 & 99 & 88 & 100 & 92 & 96 & 99 & 95 \\
\hline FSP & SED-PLA & 90 & 83 & 82 & 98 & 77 & 86 & 92 & 83 & 90 & 93 & 95 & 91 \\
\hline VLO & SED-PLA & 106 & 106 & 108 & 102 & 103 & 105 & 97 & 103 & 94 & 91 & 96 & 96 \\
\hline BFR & SED-PLA & 83 & 104 & 86 & 90 & 90 & 91 & 86 & 77 & 94 & 98 & 75 & 86 \\
\hline MRS & SED-PLA & 92 & 88 & 92 & 90 & 113 & 95 & 84 & 89 & 82 & 84 & 76 & 83 \\
\hline LMLM & SED-PLA & 86 & 81 & 88 & 84 & 87 & 85 & 72 & 76 & 82 & 75 & 80 & 77 \\
\hline MDTBS & SED-PLA & 81 & 84 & 86 & 83 & 85 & - & - & - & - & - & - & - \\
\hline MVL & SED-PLA & 81 & 81 & 84 & 81 & 51 & 76 & 116 & 89 & 76 & 84 & 80 & 89 \\
\hline POS & SED-TRH & - & - & 112 & 110 & 117 & 113 & 83 & 81 & 78 & - & - & 81 \\
\hline LMS & SED-TRH & - & 89 & 96 & 92 & - & 92 & 116 & 92 & 96 & 95 & 98 & 99 \\
\hline MUN & SED-TRH & 79 & & 81 & 90 & 73 & 81 & 92 & 97 & 91 & 99 & 91 & 94 \\
\hline JJS & SED-TRH & 112 & 95 & 97 & 139 & - & 111 & 83 & 93 & 84 & 89 & 84 & 87 \\
\hline NPB & SED-TRH & 103 & 106 & 119 & 120 & 102 & 110 & 95 & 94 & 93 & 87 & 128 & 99 \\
\hline RFS & SED-TRH & 91 & 75 & 76 & 73 & 88 & 81 & 92 & 79 & 80 & 98 & 88 & 87 \\
\hline AAR & SED-TRH & - & - & 88 & 96 & 87 & 90 & 99 & 111 & 95 & 101 & 97 & 101 \\
\hline SMDR & SED-TRH & - & 96 & 78 & 89 & - & 88 & 100 & 100 & 88 & 83 & 82 & 91 \\
\hline GG & SED-TRH & 92 & 86 & 84 & 85 & 82 & 86 & 85 & 74 & 73 & 81 & 85 & 80 \\
\hline MESS & SED-TRH & 113 & 110 & 111 & 112 & 121 & 113 & 101 & 124 & 118 & 105 & 105 & 111 \\
\hline SRGP & SED-TRH & 76 & 83 & 83 & 84 & 76 & 80 & 82 & 88 & 83 & 88 & 84 & 85 \\
\hline VLCO & SED-TRH & 89 & 94 & 89 & 89 & 86 & - & - & - & - & - & - & - \\
\hline VFG & SED-TRH & 84 & 87 & 82 & 90 & 86 & 86 & 93 & 85 & 87 & 81 & 84 & 86 \\
\hline ECB & TF-PLA & 83 & 90 & 98 & - & - & 90 & 97 & - & 102 & 90 & - & 96 \\
\hline SRS & TF-PLA & 108 & - & 116 & - & - & - & - & 126 & - & 136 & - & - \\
\hline MCSA & TF-PLA & - & 115 & 110 & - & - & 113 & - & 102 & 104 & - & 112 & 106 \\
\hline AAOS & TF-PLA & 98 & 101 & 89 & 101 & 96 & 97 & 100 & 92 & - & 99 & 100 & 98 \\
\hline ITS & TF-PLA & 92 & 104 & 104 & 101 & 107 & 102 & 84 & 93 & 86 & 83 & 77 & 85 \\
\hline MACS & TF-PLA & - & - & - & - & - & - & 93 & 105 & 103 & 95 & 96 & \\
\hline SCS & TF-PLA & 88 & 92 & 104 & 98 & & 96 & 94 & 83 & 108 & 83 & 83 & 90 \\
\hline WGF & TF-PLA & 106 & 113 & 109 & 107 & 107 & 108 & 96 & 107 & 102 & 117 & 113 & 107 \\
\hline JMBP & TF-PLA & 106 & 102 & 92 & 93 & - & 98 & 108 & 96 & 93 & - & 99 & 99 \\
\hline DMB & TF-PLA & 83 & 82 & 80 & 84 & - & 82 & - & 80 & 81 & 79 & 73 & 78 \\
\hline SMS & TF-TRH & 100 & 100 & 101 & - & - & 100 & 100 & - & 99 & 100 & 95 & 99 \\
\hline ALCLM & TF-TRH & & 126 & 144 & 136 & - & 135 & 116 & 108 & 99 & - & - & 108 \\
\hline ORS & TF-TRH & 102 & 126 & 114 & 106 & 110 & 112 & 100 & 103 & 96 & 88 & 93 & 96 \\
\hline MAO & TF-TRH & 87 & 96 & 88 & 83 & 83 & 87 & 64 & 70 & 67 & 81 & 74 & 71 \\
\hline SSP & TF-TRH & 77 & 99 & 90 & - & - & 88,67 & 88 & 87 & 87 & - & 98 & 90 \\
\hline EMPS & TF-TRH & - & - & - & - & - & - & 83 & 83 & 86 & 91 & 96 & 88 \\
\hline NVM & TF-TRH & 69 & 72 & 69 & 70 & 75 & 71 & - & 84 & 90 & - & 87 & 87 \\
\hline VLMS & TF-TRH & 76 & 86 & 92 & 81 & - & 84 & - & - & - & - & - & - \\
\hline
\end{tabular}


Valores de FC (bpm)

\begin{tabular}{|c|c|c|c|c|c|c|c|c|c|c|c|c|c|}
\hline \multirow[b]{3}{*}{ voluntárias } & \multirow[b]{3}{*}{ grupo } & \multicolumn{6}{|c|}{ SESSÃO PRÉ } & \multicolumn{6}{|c|}{ SESSÃO PÓS } \\
\hline & & \multicolumn{5}{|c|}{ minutos } & \multirow[b]{2}{*}{ média } & \multicolumn{5}{|c|}{ minutos } & \multirow[b]{2}{*}{ média } \\
\hline & & 1 & 2 & 3 & 4 & 5 & & 1 & 2 & 3 & 4 & 5 & \\
\hline MMC & SED-PLA & - & 77 & 78 & - & 79 & 77 & 75 & 73 & 71 & - & - & 73 \\
\hline MARO & SED-PLA & 68 & 68 & 68 & 68 & 66 & 68 & 70 & 66 & 70 & 70 & 75 & 70 \\
\hline RCA & SED-PLA & 75 & 78 & 78 & 78 & 77 & 77 & 77 & 78 & 75 & 75 & 79 & 77 \\
\hline MYI & SED-PLA & 67 & 65 & 64 & 65 & 68 & 66 & 72 & 72 & 70 & 75 & 72 & 72 \\
\hline FSP & SED-PLA & - & 89 & 93 & 93 & 92 & 92 & 78 & 82 & 78 & 82 & 87 & 81 \\
\hline VLO & SED-PLA & 65 & 67 & 68 & 69 & 67 & 67 & 66 & 68 & 66 & 66 & 67 & 67 \\
\hline BFR & SED-PLA & 60 & 64 & 58 & 58 & 60 & 60 & 55 & 55 & 57 & 57 & 55 & 56 \\
\hline MRS & SED-PLA & 73 & 73 & 75 & - & - & 74 & 63 & 60 & 61 & 63 & 66 & 63 \\
\hline LMLM & SED-PLA & 66 & 67 & 67 & 70 & 70 & 68 & 75 & 76 & 75 & 75 & 75 & 75 \\
\hline MDTBS & SED-PLA & 63 & 63 & 64 & 61 & 63 & 63 & 61 & 60 & 60 & 61 & 60 & 60 \\
\hline MVL & SED-PLA & 54 & 53 & 54 & 54 & 51 & 53 & 55 & 63 & 59 & 60 & 58 & 59 \\
\hline POS & SED-TRH & - & - & 60 & 61 & 61 & 61 & 54 & 53 & 54 & 55 & - & 54 \\
\hline LMS & SED-TRH & - & 55 & 55 & 57 & - & 56 & 56 & 57 & 56 & 55 & 55 & 56 \\
\hline MUN & SED-TRH & 76 & 75 & 73 & - & - & 75 & 70 & 70 & 69 & 70 & 77 & 71 \\
\hline JJS & SED-TRH & 56 & 58 & 56 & 59 & - & 57 & 61 & 63 & 61 & 60 & 65 & 62 \\
\hline NPB & SED-TRH & 55 & 60 & 55 & - & 57 & 57 & 66 & 65 & 64 & 63 & 65 & 65 \\
\hline RFS & SED-TRH & 100 & 94 & 98 & 101 & 101 & 99 & 104 & 92 & 100 & 91 & 100 & 97 \\
\hline AAR & SED-TRH & - & - & 63 & 62 & 62 & 62 & 61 & 59 & 58 & 58 & 58 & 59 \\
\hline SMDR & SED-TRH & 48 & 46 & 43 & 44 & - & 45 & 47 & 46 & 51 & - & 46 & 48 \\
\hline GG & SED-TRH & 92 & 86 & 84 & 85 & 82 & 86 & 49 & 50 & 50 & 51 & 50 & 50 \\
\hline MESS & SED-TRH & 77 & 73 & 82 & 76 & 74 & 76 & 61 & 70 & 59 & 60 & 65 & 63 \\
\hline SRGP & SED-TRH & - & 66 & 60 & 61 & 59 & 62 & 66 & 65 & 68 & 66 & 65 & 66 \\
\hline VLCO & SED-TRH & 84 & 85 & 87 & 82 & 84 & & - & - & - & - & - & - \\
\hline VFG & SED-TRH & 66 & 68 & 66 & 68 & 66 & 67 & - & 72 & 70 & 68 & 71 & 70 \\
\hline ECB & TF-PLA & 62 & 60 & 64 & - & - & 62 & 63 & 60 & 63 & - & 66 & 63 \\
\hline SRS & TF-PLA & - & 79 & 82 & 85 & - & 82 & 82 & 83 & 84 & 84 & - & 83 \\
\hline MCSA & TF-PLA & 67 & 70 & 79 & - & - & 72 & 72 & 73 & 75 & 75 & 74 & 74 \\
\hline AAOS & TF-PLA & 76 & 73 & 76 & 77 & 74 & 75 & 66 & 65 & & 65 & 66 & 66 \\
\hline ITS & TF-PLA & 69 & 69 & 72 & 68 & 67 & 69 & 66 & 67 & 67 & 66 & 67 & 67 \\
\hline MACS & TF-PLA & - & - & - & - & - & - & 60 & 60 & 60 & 61 & 63 & - \\
\hline SCS & TF-PLA & 63 & 63 & 63 & 64 & 63 & 63 & 59 & 58 & 56 & 58 & 59 & 58 \\
\hline WGF & TF-PLA & 59 & 62 & 63 & 63 & 66 & 63 & 65 & 68 & - & - & 68 & 67 \\
\hline JMBP & TF-PLA & - & - & - & - & - & 66 & 63 & 58 & 60 & 61 & 58 & 60 \\
\hline DMB & TF-PLA & 60 & 59 & 61 & 60 & 58 & 60 & 55 & 60 & 59 & 59 & 56 & 58 \\
\hline EMCB & TF-TRH & - & 78 & 78 & - & - & 78 & 72 & - & 75 & 75 & 75 & 74 \\
\hline SMS & TF-TRH & 49 & 57 & 48 & 58 & - & 53 & 52 & - & 52 & 53 & 53 & 53 \\
\hline ALCLM & TF-TRH & 68 & 67 & - & 66 & 69 & 67,5 & 60 & 59 & 59 & - & - & 59 \\
\hline ORS & TF-TRH & 62 & 64 & 61 & 62 & 63 & 62 & 62 & 61 & 61 & - & 63 & 62 \\
\hline MAO & TF-TRH & 74 & 73 & 73 & 75 & 73 & 74 & 70 & 70 & 71 & 69 & 72 & 70 \\
\hline SSP & TF-TRH & - & 66 & 65 & 66 & - & 66 & 57 & 56 & 58 & 59 & 60 & 58 \\
\hline NVM & TF-TRH & 55 & 64 & 59 & 56 & 58 & 58 & 59 & 55 & 60 & - & - & 58 \\
\hline VLMS & TF-TRH & 66 & 63 & 63 & 62 & - & 64 & - & - & - & - & - & 60 \\
\hline
\end{tabular}


Valores de FSA [ml.(min.100ml)]

\begin{tabular}{|c|c|c|c|c|c|c|c|c|c|c|c|c|c|}
\hline \multirow[b]{3}{*}{ voluntárias } & \multirow[b]{3}{*}{ grupo } & \multicolumn{6}{|c|}{ SESSÃO PRÉ } & \multicolumn{6}{|c|}{ SESSÃO PÓS } \\
\hline & & \multicolumn{5}{|c|}{ Minutos } & \multirow[b]{2}{*}{ média } & \multicolumn{5}{|c|}{ minutos } & \multirow[b]{2}{*}{ média } \\
\hline & & 1 & 2 & 3 & 4 & 5 & & 1 & 2 & 3 & 4 & 5 & \\
\hline MMC & SED-PLA & 1,6 & 1,5 & 1,7 & 2,0 & 1,4 & 1,6 & 1,1 & 1,1 & 1,0 & 1,1 & 0,9 & 1,0 \\
\hline MARO & SED-PLA & - & 2,0 & 2,0 & 1,6 & 1,7 & 1,7 & 1,3 & 1,5 & 1,2 & 1,6 & 1,6 & 1,4 \\
\hline RCA & SED-PLA & 2,0 & 2,3 & 2,0 & 2,0 & 1,9 & 1,6 & 1,4 & - & - & - & - & 1,4 \\
\hline MYI & SED-PLA & 1,5 & 1,8 & 1,8 & 1,2 & 1,1 & 1,5 & 1,2 & 1,0 & 0,9 & 1,0 & 1,0 & 1,0 \\
\hline FSP & SED-PLA & 4,9 & 4,2 & 4,5 & 5,5 & 5,1 & 4,5 & 3,1 & 3,1 & 2,5 & 3,6 & 3,8 & 3,2 \\
\hline VLO & SED-PLA & 0,4 & 0,5 & 0,3 & 0,4 & - & 0,4 & 1,3 & 1,3 & 0,7 & 1,2 & 1,1 & 1,1 \\
\hline LMLM & SED-PLA & 1,1 & 1,1 & 1,3 & 2,0 & 2,0 & 1,5 & 3,5 & 3,7 & 4,1 & 3,1 & 3,4 & 3,6 \\
\hline MDTBS & SED-PLA & - & - & - & - & - & - & 3,3 & 3,1 & 3,8 & 3,5 & 3,5 & 3,4 \\
\hline MVL & SED-PLA & 2,0 & 1,5 & 1,6 & 1,7 & 1,8 & 1,7 & 1,4 & 1,3 & 1,5 & 1,3 & 1,4 & 1,4 \\
\hline EJGV & SED-TRH & 1,0 & 1,0 & - & - & - & 1,0 & - & - & - & - & - & - \\
\hline LMS & SED-TRH & - & 1,5 & 1,4 & 1,5 & - & 1,5 & - & - & - & - & - & - \\
\hline JJS & SED-TRH & 2,0 & 2,0 & 1,2 & 1,5 & 2,0 & 1,7 & - & - & - & - & - & - \\
\hline RFS & SED-TRH & - & - & - & - & - & - & 3,3 & 1,7 & 1,9 & 2,5 & 2,2 & 2,3 \\
\hline AAR & SED-TRH & 1,6 & 1,7 & 2,5 & 2,5 & - & 1,7 & 1,7 & 1,7 & 1,6 & 1,7 & 1,7 & 1,7 \\
\hline SMDR & SED-TRH & 1,4 & 1,5 & 1,2 & 0,7 & 0,7 & 1,1 & 1,8 & 2,0 & 1,6 & 2,4 & - & 1,7 \\
\hline GG & SED-TRH & 1,0 & 1,0 & 1,4 & 1,0 & 0,9 & 1,1 & 1,3 & 1,4 & 1,6 & 1,3 & - & 1,4 \\
\hline MESS & SED-TRH & 2,9 & 3,0 & 3,3 & 2,9 & 2,7 & 3,0 & 2,0 & 2,2 & 2,3 & 2,3 & - & 2,2 \\
\hline VLCO & SED-TRH & 2,2 & 1,9 & 2,3 & 2,3 & 2,6 & 2,3 & - & - & - & - & - & - \\
\hline VFG & SED-TRH & 4,8 & 5,4 & 5,8 & 5,6 & 6,5 & 5,6 & 5,3 & 5,6 & 4,7 & 4,8 & - & 5,1 \\
\hline SM & TF-PLA & 2,1 & 2,0 & 2,5 & 2,2 & 1,9 & 2,1 & - & - & - & - & - & - \\
\hline SRS & TF-PLA & 2,0 & 2,2 & 2,3 & 2,2 & 2,0 & 2,1 & - & 2,6 & 2,8 & 2,3 & - & 2,6 \\
\hline AAOS & TF-PLA & 2,6 & 2,4 & 2,5 & 2,5 & - & 2,5 & 2,8 & 3,1 & 2,9 & 2,9 & 2,9 & 2,9 \\
\hline ITS & TF-PLA & 3,0 & 3,0 & 2,7 & 2,6 & 2,5 & 2,8 & 3,3 & 3,3 & 3,1 & 2,8 & 3,4 & 3,2 \\
\hline MACS & TF-PLA & - & - & - & - & - & - & 1,4 & 1,6 & 1,8 & 2,0 & 1,6 & 1,7 \\
\hline SCS & TF-PLA & 4,4 & 4,9 & 4,5 & 2,8 & 2,3 & 3,8 & 6,4 & 5,6 & 4,7 & 5,8 & - & 5,6 \\
\hline WGF & TF-PLA & 1,4 & 1,9 & 1,6 & 1,8 & - & 1,5 & 5,7 & 5,4 & 5,8 & - & - & 5,6 \\
\hline JMBP & TF-PLA & - & - & - & - & - & - & 2,0 & 2,0 & 2,5 & 2,1 & - & 2,1 \\
\hline DMB & TF-PLA & 0,9 & 1,4 & 0,7 & - & - & 1,0 & 1,4 & 1,6 & 1,7 & 1,9 & - & 1,6 \\
\hline
\end{tabular}


ANEXO F - Dados dos estágios dos exercícios estáticos com "handgrip"

Valores de ANSP (impulsos/min)

\begin{tabular}{|c|c|c|c|c|c|c|c|c|c|}
\hline & & \multicolumn{4}{|c|}{ SESSÃO PRÉ } & \multicolumn{4}{|c|}{ SESSÃO PÓS } \\
\hline Voluntárias & Grupo & REP & EE & OCLU & REC & REP & EE & OCLU & REC \\
\hline $\mathrm{MMC}$ & SED-PLA & 55 & 52 & 53 & 47 & 58 & 54 & 59 & 47 \\
\hline MARO & SED-PLA & 26 & 35 & 42 & 26 & 30 & 35 & 43 & 33 \\
\hline RCA & SED-PLA & 53 & 61 & 65 & 57 & 53 & 51 & 57 & 50 \\
\hline MYI & SED-PLA & 41 & 43 & 42 & 40 & 42 & 46 & 44 & 49 \\
\hline FSP & SED-PLA & 35 & 46 & 50 & 54 & 35 & 56 & 53 & 45 \\
\hline VLO & SED-PLA & 42 & 50 & 43 & 45 & 35 & 43 & 41 & 37 \\
\hline MRS & SED-PLA & 42 & 50 & 56 & 50 & 40 & 44 & 43 & 42 \\
\hline MDTBS & SED-PLA & 47 & 55 & 54 & 44 & 46 & 55 & 50 & 37 \\
\hline MVL & SED-PLA & 35 & 40 & 32 & 34 & 29 & 36 & 37 & 33 \\
\hline POS & SED-TRH & 43 & 49 & 50 & 46 & 43 & 52 & 50 & 47 \\
\hline EJGV & SED-TRH & 50 & 50 & 47 & 51 & 35 & 42 & 42 & 40 \\
\hline LMS & SED-TRH & 29 & 37 & 34 & 25 & 41 & 46 & 55 & 46 \\
\hline JJS & SED-TRH & 32 & 39 & 36 & 36 & 34 & 35 & 39 & 33 \\
\hline NPB & SED-TRH & 38 & 44 & 41 & 41 & 29 & 32 & 33 & 31 \\
\hline RFS & SED-TRH & 40 & 41 & 47 & 35 & 35 & 44 & 44 & 39 \\
\hline AAR & SED-TRH & 57 & 56 & 58 & 59 & 43 & 38 & 43 & 43 \\
\hline MESS & SED-TRH & 37 & 34 & 43 & 41 & 42 & 48 & 45 & 32 \\
\hline SRGP & SED-TRH & 55 & 65 & 55 & 48 & 49 & 57 & 53 & 44 \\
\hline ECB & TF-PLA & 47 & 58 & 57 & 60 & 27 & 35 & 38 & 29 \\
\hline SRS & TF-PLA & 25 & 36 & 43 & 26 & 33 & 38 & 47 & 31 \\
\hline ITS & TF-PLA & 35 & 34 & 36 & 35 & 40 & 54 & 46 & 42 \\
\hline WGF & TF-PLA & 38 & 45 & 48 & 34 & 41 & 50 & 40 & 38 \\
\hline JMBP & TF-PLA & 46 & 57 & 53 & 45 & 40 & 42 & 45 & 43 \\
\hline SMS & TF-TRH & 49 & 42 & 55 & 48 & 55 & 53 & 56 & 50 \\
\hline ALCLM & TF-TRH & 44 & 48 & 46 & 43 & 37 & 44 & 42 & 37 \\
\hline ORS & TF-TRH & 36 & 32 & 33 & 32 & 31 & 33 & 35 & 36 \\
\hline MAO & TF-TRH & 30 & 34 & 40 & 34 & 36 & 32 & 28 & 31 \\
\hline EMPS & TF-TRH & 42 & 51 & 45 & 38 & 33 & 43 & 40 & 34 \\
\hline
\end{tabular}


Valores de PAS ( $\mathrm{mm} \mathrm{Hg}$ )

\begin{tabular}{|c|c|c|c|c|c|c|c|c|c|}
\hline & & \multicolumn{4}{|c|}{ SESSÃO PRÉ } & \multicolumn{4}{|c|}{ SESSÃO PÓS } \\
\hline Voluntárias & Grupo & REP & $\mathrm{EE}$ & OCLU & REC & REP & $\mathrm{EE}$ & OCLU & REC \\
\hline MMC & SED-PLA & 132 & 152 & 140 & 125 & 128 & 158 & 155 & 122 \\
\hline MARO & SED-PLA & 171 & 190 & 167 & 156 & 164 & 177 & 162 & 156 \\
\hline RCA & SED-PLA & 156 & 167 & 136 & 156 & 161 & 178 & 188 & 175 \\
\hline MYI & SED-PLA & 175 & 169 & 164 & 160 & 159 & 168 & 157 & 155 \\
\hline FSP & SED-PLA & 132 & 146 & 141 & 136 & 156 & 177 & 177 & 150 \\
\hline VLO & SED-PLA & 166 & 173 & 187 & 175 & 146 & 161 & 163 & 153 \\
\hline MRS & SED-PLA & 134 & 155 & 154 & 134 & 131 & 149 & 138 & 129 \\
\hline LMLM & SED-PLA & 137 & 146 & 137 & 142 & 148 & 167 & 145 & 116 \\
\hline MDTBS & SED-PLA & 138 & 152 & 168 & 143 & 137 & 149 & 167 & 132 \\
\hline MVL & SED-PLA & 142 & 134 & 142 & 137 & 151 & 156 & 148 & 135 \\
\hline POS & SED-TRH & 150 & 171 & 164 & 153 & 153 & 166 & 169 & 150 \\
\hline EJGV & SED-TRH & 154 & 165 & 161 & 154 & 145 & 167 & 154 & 149 \\
\hline LMS & SED-TRH & 157 & 173 & 155 & 173 & 168 & 178 & 164 & 168 \\
\hline JJS & SED-TRH & 171 & 176 & 183 & 178 & 140 & 154 & 158 & 140 \\
\hline NPB & SED-TRH & 164 & 179 & 187 & 157 & 169 & 174 & 177 & 164 \\
\hline RFS & SED-TRH & 111 & 116 & 120 & 112 & 118 & 140 & 126 & 103 \\
\hline AAR & SED-TRH & 150 & 165 & 171 & 144 & 160 & 161 & 157 & 159 \\
\hline SMDR & SED-TRH & 141 & 141 & 137 & 136 & 142 & 164 & 146 & 147 \\
\hline GG & SED-TRH & 148 & 161 & 149 & 142 & 141 & 149 & 156 & 137 \\
\hline MESS & SED-TRH & 170 & 167 & 172 & 172 & 161 & 161 & 154 & 157 \\
\hline SRGP & SED-TRH & 137 & 179 & 170 & 160 & 148 & 158 & 155 & 147 \\
\hline ECB & TF-PLA & 151 & 163 & 164 & 145 & 156 & 176 & 169 & 158 \\
\hline SRS & TF-PLA & 172 & 170 & 177 & 164 & 134 & 162 & 176 & 146 \\
\hline ITS & TF-PLA & 179 & 183 & 186 & 180 & 159 & 160 & 163 & 161 \\
\hline SCS & TF-PLA & 129 & 141 & 142 & 134 & 134 & 139 & 143 & 135 \\
\hline WGF & TF-PLA & 176 & 185 & 191 & 185 & 160 & 175 & 182 & 162 \\
\hline JMBP & TF-PLA & 152 & 178 & 190 & 154 & 145 & 160 & 184 & 150 \\
\hline DMB & TF-PLA & 124 & 162 & 154 & 135 & 125 & 141 & 131 & 136 \\
\hline SMS & TF-TRH & 164 & 158 & 168 & 168 & 159 & 168 & 171 & 153 \\
\hline ALCLM & TF-TRH & 194 & 190 & 194 & 194 & 186 & 193 & 188 & 183 \\
\hline ORS & TF-TRH & 176 & 177 & 186 & 179 & 174 & 184 & 178 & 174 \\
\hline MAO & TF-TRH & 134 & 138 & 134 & 137 & 110 & 111 & 102 & 99 \\
\hline SSP & TF-TRH & 132 & 152 & 167 & 124 & 152 & 171 & 167 & 156 \\
\hline EMPS & TF-TRH & 140 & 167 & 160 & 150 & 151 & 175 & 165 & 153 \\
\hline NVM & TF-TRH & 131 & 145 & 145 & 131 & 145 & 165 & 176 & 148 \\
\hline
\end{tabular}


Valores de PAD $(\mathrm{mm} \mathrm{Hg})$

\begin{tabular}{|c|c|c|c|c|c|c|c|c|c|}
\hline & & \multicolumn{4}{|c|}{ SESSÃO PRÉ } & \multicolumn{4}{|c|}{ SESSÃO PÓS } \\
\hline Voluntárias & Grupo & REP & EE & OCLU & REC & REP & EE & OCLU & REC \\
\hline MMC & SED-PLA & 59 & 78 & 63 & 60 & 67 & 120 & 81 & 69 \\
\hline MARO & SED-PLA & 76 & 88 & 79 & 72 & 80 & 93 & 75 & 76 \\
\hline RCA & SED-PLA & 84 & 105 & 88 & 90 & 88 & 93 & 109 & 96 \\
\hline MYI & SED-PLA & 95 & 87 & 90 & 84 & 82 & 85 & 87 & 82 \\
\hline FSP & SED-PLA & 68 & 74 & 76 & 64 & 70 & 89 & 84 & 75 \\
\hline VLO & SED-PLA & 83 & 91 & 88 & 85 & 81 & 86 & 92 & 82 \\
\hline MRS & SED-PLA & 77 & 95 & 93 & 73 & 67 & 79 & 76 & 74 \\
\hline LMLM & SED-PLA & 63 & 75 & 69 & 64 & 64 & 81 & 72 & 63 \\
\hline MDTBS & SED-PLA & 66 & 81 & 77 & 68 & 62 & 69 & 70 & 61 \\
\hline MVL & SED-PLA & 76 & 82 & 81 & 82 & 77 & 74 & 76 & 72 \\
\hline POS & SED-TRH & 77 & 88 & 93 & 79 & 68 & 85 & 81 & 72 \\
\hline EJGV & SED-TRH & 76 & 82 & 80 & 75 & 78 & 96 & 84 & 73 \\
\hline LMS & SED-TRH & 95 & 106 & 101 & 92 & 76 & 88 & 100 & 74 \\
\hline JJS & SED-TRH & 96 & 116 & 129 & 108 & 72 & 82 & 76 & 74 \\
\hline NPB & SED-TRH & 85 & 108 & 116 & 97 & 81 & 97 & 88 & 81 \\
\hline SMDR & SED-TRH & 64 & 71 & 68 & 67 & 66 & 88 & 71 & 68 \\
\hline GG & SED-TRH & 66 & 71 & 66 & 66 & 82 & 84 & 79 & 81 \\
\hline MESS & SED-TRH & 95 & 99 & 100 & 94 & 71 & 88 & 80 & 77 \\
\hline SRGP & SED-TRH & 67 & 107 & 87 & 72 & 63 & 71 & 71 & 63 \\
\hline ECB & TF-PLA & 76 & 92 & 84 & 69 & 79 & 103 & 88 & 80 \\
\hline SRS & TF-PLA & 95 & 103 & 94 & 93 & 78 & 94 & 97 & 80 \\
\hline ITS & TF-PLA & 84 & 91 & 95 & 89 & 78 & 84 & 86 & 82 \\
\hline SCS & TF-PLA & 71 & 77 & 75 & 69 & 76 & 76 & 70 & 72 \\
\hline WGF & TF-PLA & 94 & 118 & 116 & 99 & 83 & 102 & 100 & 87 \\
\hline JMBP & TF-PLA & 79 & 109 & 115 & 79 & 75 & 92 & 104 & 76 \\
\hline DMB & TF-PLA & 67 & 107 & 76 & 73 & 66 & 79 & 74 & 69 \\
\hline SMS & TF-TRH & 85 & 92 & 88 & 83 & 82 & 91 & 88 & 82 \\
\hline ALCLM & TF-TRH & 110 & 120 & 127 & 113 & 87 & 109 & 98 & 89 \\
\hline ORS & TF-TRH & 96 & 105 & 106 & 89 & 77 & 98 & 83 & 75 \\
\hline MAO & TF-TRH & 71 & 75 & 70 & 66 & 60 & 62 & 55 & 59 \\
\hline SSP & TF-TRH & 62 & 79 & 67 & 61 & 66 & 83 & 81 & 66 \\
\hline EMPS & TF-TRH & 73 & 80 & 75 & 73 & 74 & 101 & 80 & 70 \\
\hline
\end{tabular}


Valores de PAM (mm Hg)

\begin{tabular}{|c|c|c|c|c|c|c|c|c|c|}
\hline & & \multicolumn{4}{|c|}{ SESSÃO PRÉ } & \multicolumn{4}{|c|}{ SESSÃO PÓS } \\
\hline Voluntárias & Grupo & REP & $\mathrm{EE}$ & OCLU & REC & REP & EE & OCLU & REC \\
\hline MMC & SED-PLA & 79 & 96 & 84 & 75 & 79 & 131 & 102 & 85 \\
\hline MARO & SED-PLA & 96 & 109 & 97 & 90 & 101 & 112 & 94 & 90 \\
\hline MYI & SED-PLA & 116 & 104 & 123 & 104 & 99 & 102 & 99 & 96 \\
\hline FSP & SED-PLA & 91 & 95 & 94 & 85 & 91 & 109 & 100 & 90 \\
\hline VLO & SED-PLA & 106 & 110 & 109 & 108 & 100 & 105 & 107 & 103 \\
\hline MRS & SED-PLA & 96 & 107 & 111 & 91 & 84 & 94 & 90 & 88 \\
\hline LMLM & SED-PLA & 84 & 89 & 83 & 85 & 81 & 103 & 90 & 81 \\
\hline MDTBS & SED-PLA & 85 & 105 & 93 & 89 & 80 & 92 & 96 & 75 \\
\hline MVL & SED-PLA & 96 & 99 & 99 & 99 & 99 & 89 & 92 & 85 \\
\hline POS & SED-TRH & 105 & 114 & 121 & 102 & 86 & 109 & 97 & 87 \\
\hline LMS & SED-TRH & 99 & 117 & 112 & 107 & 101 & 110 & 100 & 98 \\
\hline JJS & SED-TRH & 125 & 134 & 152 & 128 & 82 & 97 & 98 & 93 \\
\hline NPB & SED-TRH & 102 & 134 & 142 & 110 & 104 & 114 & 108 & 96 \\
\hline RFS & SED-TRH & 82 & 87 & 89 & 86 & 87 & 106 & 86 & 83 \\
\hline AAR & SED-TRH & 91 & 106 & 106 & 98 & 94 & 99 & 98 & 102 \\
\hline SMDR & SED-TRH & 88 & 87 & 86 & 81 & 93 & 108 & 90 & 96 \\
\hline GG & SED-TRH & 88 & 93 & 84 & 87 & 90 & 99 & 98 & 86 \\
\hline MESS & SED-TRH & 112 & 117 & 117 & 115 & 105 & 112 & 110 & 106 \\
\hline SRGP & SED-TRH & 84 & 126 & 102 & 86 & 82 & 100 & 94 & 91 \\
\hline VFG & SED-TRH & 94 & 113 & 99 & 88 & 84 & 117 & 106 & 89 \\
\hline SRS & TF-PLA & 117 & 119 & 108 & 105 & 99 & 109 & 112 & 96 \\
\hline ITS & TF-PLA & 106 & 112 & 121 & 108 & 97 & 108 & 101 & 102 \\
\hline SCS & TF-PLA & 82 & 99 & 101 & 91 & 89 & 98 & 85 & 87 \\
\hline WGF & TF-PLA & 116 & 144 & 128 & 114 & 105 & 122 & 123 & 107 \\
\hline JMBP & TF-PLA & 98 & 125 & 134 & 103 & 99 & 111 & 119 & 100 \\
\hline DMB & TF-PLA & 77 & 130 & 99 & 92 & 88 & 102 & 93 & 89 \\
\hline SMS & TF-TRH & 96 & 107 & 107 & 99 & 103 & 111 & 104 & 98 \\
\hline SLCLM & TF-TRH & 129 & 158 & 156 & 132 & 107 & 129 & 112 & 102 \\
\hline ORS & TF-TRH & 120 & 129 & 133 & 117 & 97 & 116 & 110 & 97 \\
\hline MAO & TF-TRH & 83 & 91 & 76 & 86 & 74 & 75 & 69 & 72 \\
\hline SSP & TF-TRH & 84 & 54 & 86 & 85 & 87 & 96 & 104 & 89 \\
\hline NVM & TF-TRH & 81 & 97 & 89 & 85 & 96 & 116 & 112 & 91 \\
\hline
\end{tabular}


Valores de FC (bpm)

\begin{tabular}{|c|c|c|c|c|c|c|c|c|c|}
\hline & & \multicolumn{4}{|c|}{ SESSÃO PRÉ } & \multicolumn{4}{|c|}{ SESSÃO PÓS } \\
\hline Voluntárias & Grupos & REP & EE & OCLU & REC & REP & EE & OCLU & REC \\
\hline MMC & SED-PLA & 76 & 80 & 72 & 74 & 71 & 75 & 65 & 69 \\
\hline MARO & SED-PLA & 65 & 75 & 72 & 65 & 69 & 76 & 66 & 68 \\
\hline RCA & SED-PLA & 74 & 80 & 80 & 76 & 74 & 80 & 85 & 76 \\
\hline MYI & SED-PLA & 67 & 69 & 70 & 69 & 75 & 76 & 78 & 77 \\
\hline FSP & SED-PLA & 89 & 94 & 92 & 85 & 77 & 90 & 80 & 80 \\
\hline VLO & SED-PLA & 67 & 68 & 73 & 68 & 67 & 69 & 69 & 69 \\
\hline MRS & SED-PLA & 61 & 78 & 75 & 69 & 57 & 73 & 65 & 60 \\
\hline LMLM & SED-PLA & 65 & 68 & 67 & 66 & 69 & 79 & 77 & 69 \\
\hline MDTBS & SED-PLA & 60 & 65 & 63 & 59 & 71 & 79 & 76 & 70 \\
\hline MVL & SED-PLA & 65 & 70 & 69 & 70 & 62 & 68 & 65 & 64 \\
\hline POS & SED-TRH & 55 & 58 & 61 & 57 & 56 & 63 & 61 & 58 \\
\hline LMS & SED-TRH & 64 & 76 & 63 & 52 & 54 & 63 & 57 & 54 \\
\hline JJS & SED-TRH & 58 & 81 & 57 & 60 & 59 & 66 & 57 & 56 \\
\hline NPB & SED-TRH & 57 & 61 & 63 & 58 & 65 & 73 & 68 & 67 \\
\hline RFS & SED-TRH & 89 & 94 & 93 & 90 & 89 & 104 & 88 & 90 \\
\hline AAR & SED-TRH & 66 & 80 & 77 & 70 & 60 & 63 & 62 & 61 \\
\hline SMDR & SED-TRH & 45 & 47 & 43 & 45 & 49 & 69 & 46 & 67 \\
\hline GG & SED-TRH & 56 & 66 & 60 & 58 & 50 & 57 & 51 & 49 \\
\hline MESS & SED-TRH & 67 & 75 & 75 & 73 & 58 & 65 & 60 & 66 \\
\hline SRGP & SED-TRH & 57 & 83 & 72 & 62 & 64 & 72 & 63 & 64 \\
\hline VFG & SED-TRH & 64 & 74 & 66 & 63 & 65 & 74 & 65 & 66 \\
\hline SRS & TF-PLA & 76 & 81 & 73 & 75 & 79 & 87 & 75 & 81 \\
\hline ITS & TF-PLA & 69 & 73 & 72 & 70 & 67 & 68 & 69 & 68 \\
\hline SCS & TF-PLA & 63 & 65 & 60 & 63 & 56 & 63 & 59 & 59 \\
\hline WGF & TF-PLA & 65 & 90 & 62 & 64 & 67 & 71 & 63 & 65 \\
\hline DMB & TF-PLA & 60 & 86 & 72 & 66 & 53 & 66 & 59 & 55 \\
\hline SMS & TF-TRH & 47 & 57 & 50 & 48 & 53 & 59 & 57 & 57 \\
\hline ALCLM & TF-TRH & 61 & 76 & 73 & 64 & 57 & 61 & 61 & 57 \\
\hline ORS & TF-TRH & 63 & 71 & 72 & 65 & 60 & 73 & 62 & 62 \\
\hline MAO & TF-TRH & 71 & 76 & 74 & 73 & 72 & 75 & 71 & 71 \\
\hline NVM & TF-TRH & 57 & 69 & 63 & 60 & 54 & 66 & 63 & 55 \\
\hline
\end{tabular}


Valores de FSA [ml(min.100ml)] nas sessões PRÉ e PÓS durante estágios dos exercícios estáticos com "handgrip"

\begin{tabular}{c|c|c|c|c|c|c|c|c|c}
\hline & & \multicolumn{3}{|c|}{ SESSÃO PRÉ } & \multicolumn{3}{c}{ SESSÃO PÓS } \\
\hline Voluntárias & Grupos & REP & EE & OCLU & REC & REP & EE & OCLU & REC \\
\hline MMC & SED-PLA & 1,6 & 1,5 & 1,4 & 1,3 & 1,1 & 1,3 & 1,3 & 1,0 \\
\hline MARO & SED-PLA & 1,7 & 2,5 & 3,5 & 3,5 & 1,1 & 1,0 & 0,8 & 1,0 \\
\hline RCA & SED-PLA & 1,4 & 1,9 & 1,9 & 1,9 & 2,2 & 1,3 & 1,8 & 1,6 \\
\hline MYI & SED-PLA & 3,4 & 2,3 & 2,6 & 2,9 & 1,5 & 2,0 & 0,9 & 0,9 \\
\hline FSP & SED-PLA & 5,6 & 6,7 & 5,1 & 3,7 & 2,8 & 3,8 & 4,3 & 3,8 \\
\hline LMLM & SED-PLA & 2,0 & 1,4 & 1,3 & 0,9 & 3,8 & 4,6 & 3,3 & 2,5 \\
\hline MVL & SED-PLA & 5,3 & 5,0 & 4,7 & 4,5 & 1,6 & 4,6 & 3,4 & 4,3 \\
\hline POS & SED-TRH & 3,5 & 3,6 & 3,8 & 3,9 & 1,7 & 2,3 & 2,2 & 1,6 \\
\hline EJGV & SED-TRH & 1,0 & 1,1 & 1,2 & 1,1 & 1,4 & 1,5 & 1,7 & 1,5 \\
\hline LMS & SED-TRH & 1,5 & 1,7 & 1,6 & 1,3 & - & - & - & - \\
\hline JJS & SED-TRH & 1,6 & 1,8 & 1,7 & 1,8 & - & - & - & - \\
\hline AAR & SED-TRH & 2,4 & 3,4 & 3,4 & 2,4 & 2,5 & 2,1 & 1,8 & 1,8 \\
\hline SMDR & SED-TRH & 1,7 & 0,8 & 0,7 & 0,9 & 2,4 & 4,5 & 1,3 & 2,2 \\
\hline GG & SED-TRH & 1,9 & 1,8 & 1,3 & 1,1 & 3 & 1,9 & 1,9 & 1,7 \\
\hline MESS & SED-TRH & 2,4 & 2,5 & 1,7 & 1,2 & 4,2 & 3,6 & 3,5 & 3,1 \\
\hline ECB & TF-PLA & 2,0 & 2,4 & 1,9 & 1,8 & 2,4 & 3,1 & 2,4 & 2,5 \\
\hline SRS & TF-PLA & 2,3 & 3,1 & 2,9 & 1,9 & 3,5 & 4,0 & 2,6 & 2,5 \\
\hline ITS & TF-PLA & 3,3 & 3,2 & 2,8 & 2,9 & 3,5 & 3,8 & 3,8 & 3,6 \\
\hline SMS & TF-TRH & 0,6 & 0,8 & 0,7 & 1,1 & 0,8 & 1,0 & 1,3 & 0,7 \\
\hline ALCLM & TF-TRH & 1,8 & 1,9 & 2,1 & 2,0 & 2,3 & 1,9 & 1,7 & 1,8 \\
\hline EMPS & TF-TRH & 1,4 & 2,5 & 1,6 & 1,1 & 2,8 & 5,0 & 2,8 & 2,0 \\
\hline NVM & TF-TRH & 3,2 & 3,0 & 3,8 & 3,2 & 1,9 & 2,8 & 3,0 & 1,6 \\
\hline & & & & & & & & \\
\hline & & & & & & \\
\hline
\end{tabular}


ANEXO G - Dados dos estágios do exercício dinâmico com "handgrip"

Valores de ANSP (impulsos/min)

\begin{tabular}{|c|c|c|c|c|c|c|c|c|c|}
\hline & & \multicolumn{4}{|c|}{ SESSÃO PRÉ } & \multicolumn{4}{|c|}{ SESSÃO PÓS } \\
\hline Voluntárias & Grupos & REP & ED & OCLU & REC & REP & ED & OCLU & REC \\
\hline MMC & SED-PLA & 57 & 63 & 61 & 69 & 46 & 56 & 59 & 58 \\
\hline MARO & SED-PLA & 21 & 30 & 28 & 25 & 26 & 40 & 36 & 25 \\
\hline FSP & SED-PLA & 41 & 48 & 45 & 48 & 39 & 46 & 42 & 37 \\
\hline MRS & SED-PLA & 47 & 42 & 44 & 47 & 39 & 39 & 45 & 38 \\
\hline MDTBS & SED-PLA & 41 & 47 & 44 & 48 & 37 & 37 & 45 & 35 \\
\hline MVL & SED-PLA & 40 & 40 & 42 & 43 & 37 & 37 & 39 & 38 \\
\hline POS & SED-TRH & 43 & 47 & 35 & 39 & 47 & 56 & 46 & 45 \\
\hline EJGV & SED-TRH & 39 & 40 & 39 & 38 & 36 & 41 & 44 & 39 \\
\hline LMS & SED-TRH & 29 & 36 & 33 & 26 & 39 & 39 & 49 & 40 \\
\hline JJS & SED-TRH & 32 & 35 & 39 & 29 & 37 & 38 & 31 & 31 \\
\hline NPB & SED-TRH & 35 & 36 & 34 & 32 & 32 & 33 & 37 & 32 \\
\hline MLC & SED-TRH & 24 & 25 & 27 & 24 & 33 & 36 & 36 & 35 \\
\hline RFS & SED-TRH & 40 & 39 & 50 & 41 & 31 & 36 & 31 & 34 \\
\hline AAR & SED-TRH & 57 & 61 & 69 & 56 & 35 & 39 & 43 & 42 \\
\hline MESS & SED-TRH & 34 & 36 & 32 & 32 & 36 & 42 & 41 & 37 \\
\hline SRGP & SED-TRH & 55 & 63 & 66 & 61 & 37 & 51 & 42 & 52 \\
\hline VFG & SED-TRH & 20 & 20 & 20 & 20 & 20 & 20 & 20 & 20 \\
\hline SRS & TF-PLA & 26 & 36 & 32 & 26 & 28 & 34 & 33 & 40 \\
\hline ITS & TF-PLA & 37 & 36 & 42 & 32 & 36 & 43 & 41 & 42 \\
\hline WGF & TF-PLA & 42 & 46 & 40 & 37 & 38 & 43 & 43 & 39 \\
\hline JMBP & TF-PLA & 38 & 41 & 51 & 47 & 48 & 53 & 53 & 48 \\
\hline SMS & TF-TRH & 50 & 53 & 53 & 52 & 61 & 54 & 53 & 46 \\
\hline ALCLM & TF-TRH & 46 & 52 & 48 & 45 & 42 & 42 & 39 & 39 \\
\hline ORS & TF-TRH & 32 & 29 & 29 & 32 & 27 & 27 & 33 & 32 \\
\hline MAO & TF-TRH & 30 & 37 & 43 & 33 & 35 & 39 & 34 & 43 \\
\hline EMPS & TF-TRH & 37 & 42 & 40 & 39 & 28 & 38 & 36 & 34 \\
\hline
\end{tabular}


Valores de PAS (impulsos/min)

\begin{tabular}{|c|c|c|c|c|c|c|c|c|c|}
\hline & & \multicolumn{4}{|c|}{ SESSÃO PRÉ } & \multicolumn{4}{|c|}{ SESSÃO PÓS } \\
\hline Voluntárias & & REP & ED & OCLU & REC & REP & ED & OCLU & REC \\
\hline MMC & SED-PLA & 135 & 149 & 137 & 132 & 133 & 143 & 139 & 128 \\
\hline MARO & SED-PLA & 162 & 170 & 180 & 163 & 160 & 179 & 158 & 158 \\
\hline RCA & SED-PLA & 146 & 162 & 164 & 148 & 167 & 173 & 187 & 178 \\
\hline MYI & SED-PLA & 163 & 161 & 152 & 167 & 157 & 161 & 164 & 166 \\
\hline FSP & SED-PLA & 149 & 149 & 145 & 141 & 159 & 165 & 162 & 160 \\
\hline VLO & SED-PLA & 165 & 175 & 175 & 173 & 151 & 159 & 162 & 156 \\
\hline MRS & SED-PLA & 140 & 149 & 138 & 134 & 132 & 138 & 124 & 126 \\
\hline LMLM & SED-PLA & 128 & 154 & 142 & 136 & 147 & 151 & 139 & 144 \\
\hline MDTBS & SED-PLA & 136 & 148 & 150 & 138 & 145 & 147 & 153 & 142 \\
\hline MVL & SED-PLA & 149 & 153 & 149 & 151 & 149 & 152 & 147 & 155 \\
\hline POS & SED-TRH & 153 & 169 & 159 & 166 & 150 & 171 & 166 & 147 \\
\hline EJGV & SED-TRH & 153 & 158 & 171 & 167 & 135 & 156 & 150 & 137 \\
\hline LMS & SED-TRH & 164 & 171 & 178 & 172 & 168 & 184 & 164 & 169 \\
\hline JJS & SED-TRH & 171 & 179 & 179 & 181 & 142 & 155 & 156 & 147 \\
\hline NPB & SED-TRH & 160 & 172 & 175 & 161 & 157 & 173 & 164 & 167 \\
\hline RFS & SED-TRH & 104 & 113 & 119 & 113 & 120 & 126 & 117 & 111 \\
\hline AAR & SED-TRH & 152 & 165 & 162 & 157 & 160 & 163 & 165 & 158 \\
\hline SMDR & SED-TRH & 141 & 139 & 154 & 146 & 145 & 154 & 164 & 138 \\
\hline GG & SED-TRH & 143 & 148 & 144 & 142 & 145 & 150 & 141 & 142 \\
\hline MESS & SED-TRH & 168 & 171 & 173 & 162 & 161 & 155 & 160 & 154 \\
\hline SRGP & SED-TRH & 138 & 163 & 134 & 141 & 135 & 169 & 158 & 142 \\
\hline VFG & SED-TRH & 143 & 147 & 157 & 144 & 137 & 161 & 161 & 125 \\
\hline ECB & TF-PLA & 160 & 167 & 169 & 144 & 162 & 167 & 162 & 154 \\
\hline SRS & TF-PLA & 150 & 174 & 172 & 162 & 131 & 166 & 176 & 143 \\
\hline MCSA & TF-PLA & 178 & 179 & 176 & 174 & 166 & 161 & 153 & 146 \\
\hline ITS & TF-PLA & 175 & 168 & 175 & 172 & 150 & 155 & 155 & 148 \\
\hline SCS & TF-PLA & 127 & 135 & 125 & 122 & 135 & 135 & 144 & 133 \\
\hline WGF & TF-PLA & 178 & 188 & 187 & 180 & 171 & 178 & 185 & 177 \\
\hline JMBP & TF-PLA & 158 & 171 & 174 & 149 & 153 & 162 & 165 & 152 \\
\hline DMB & TF-PLA & 132 & 161 & 137 & 133 & 129 & 142 & 136 & 127 \\
\hline SMS & TF-TRH & 158 & 158 & 162 & 162 & 159 & 165 & 177 & 166 \\
\hline ORS & TF-TRH & 179 & 178 & 176 & 180 & 166 & 177 & 177 & 175 \\
\hline SSP & TF-TRH & 126 & 140 & 133 & 123 & 157 & 168 & 177 & 152 \\
\hline EMPS & TF-TRH & 148 & 177 & 179 & 138 & 154 & 160 & 171 & 155 \\
\hline NVM & TF-TRH & 132 & 153 & 154 & 141 & 149 & 173 & 154 & 151 \\
\hline
\end{tabular}


Valores de PAD (mm Hg)

\begin{tabular}{|c|c|c|c|c|c|c|c|c|c|}
\hline & & \multicolumn{4}{|c|}{ SESSÃO PRÉ } & \multicolumn{4}{|c|}{ SESSÃO PÓS } \\
\hline Voluntárias & & REP & ED & OCLU & REC & REP & ED & OCLU & REC \\
\hline MMC & SED-PLA & 64 & 74 & 70 & 63 & 71 & 87 & 83 & 68 \\
\hline MARO & SED-PLA & 78 & 90 & 82 & 76 & 75 & 92 & 75 & 76 \\
\hline RCA & SED-PLA & 82 & 92 & 91 & 77 & 90 & 93 & 95 & 90 \\
\hline MYI & SED-PLA & 85 & 88 & 87 & 85 & 84 & 84 & 88 & 89 \\
\hline FSP & SED-PLA & 67 & 80 & 76 & 79 & 72 & 82 & 74 & 75 \\
\hline VLO & SED-PLA & 87 & 86 & 92 & 83 & 83 & 92 & 86 & 80 \\
\hline MRS & SED-PLA & 77 & 81 & 79 & 78 & 69 & 74 & 76 & 68 \\
\hline LMLM & SED-PLA & 65 & 78 & 63 & 61 & 65 & 74 & 66 & 63 \\
\hline MDTBS & SED-PLA & 69 & 80 & 74 & 68 & 77 & 75 & 68 & 72 \\
\hline MVL & SED-PLA & 76 & 79 & 80 & 79 & 71 & 75 & 86 & 73 \\
\hline POS & SED-TRH & 76 & 82 & 82 & 75 & 71 & 88 & 76 & 71 \\
\hline EJGV & SED-TRH & 80 & 79 & 78 & 77 & 70 & 83 & 74 & 69 \\
\hline LMS & SED-TRH & 80 & 101 & 101 & 109 & 75 & 103 & 82 & 72 \\
\hline JJS & SED-TRH & 92 & 105 & 115 & 110 & 80 & 84 & 80 & 78 \\
\hline NPB & SED-TRH & 95 & 85 & 85 & 82 & 76 & 90 & 85 & 85 \\
\hline RFS & SED-TRH & 55 & 57 & 66 & 60 & 69 & 75 & 68 & 60 \\
\hline AAR & SED-TRH & 81 & 82 & 81 & 77 & 82 & 83 & 85 & 81 \\
\hline SMDR & SED-TRH & 62 & 67 & 74 & 66 & 70 & 90 & 81 & 70 \\
\hline GG & SED-TRH & 63 & 71 & 67 & 63 & 66 & 73 & 68 & 66 \\
\hline MESS & SED-TRH & 97 & 99 & 94 & 90 & 81 & 86 & 87 & 89 \\
\hline SRGP & SED-TRH & 68 & 85 & 76 & 65 & 76 & 83 & 70 & 68 \\
\hline VFG & SED-TRH & 70 & 83 & 84 & 71 & 72 & 95 & 92 & 65 \\
\hline ECB & TF-PLA & 84 & 88 & 94 & 73 & 82 & 85 & 78 & 76 \\
\hline RSR & TF-PLA & 90 & 99 & 88 & 94 & 78 & 89 & 88 & 79 \\
\hline MCSA & TF-PLA & 100 & 103 & 98 & 97 & 97 & 102 & 104 & 94 \\
\hline ITS & TF-PLA & 82 & 85 & 85 & 87 & 74 & 78 & 79 & 74 \\
\hline SCS & TF-PLA & 66 & 70 & 69 & 68 & 71 & 77 & 74 & 70 \\
\hline WGF & TF-PLA & 98 & 105 & 98 & 94 & 90 & 106 & 101 & 91 \\
\hline JMBP & TF-PLA & 83 & 101 & 86 & 80 & 81 & 86 & 84 & 78 \\
\hline DMB & TF-PLA & 76 & 88 & 80 & 69 & 69 & 74 & 71 & 71 \\
\hline SMS & TF-TRH & 84 & 91 & 85 & 85 & 84 & 89 & 90 & 81 \\
\hline ALCLM & TF-TRH & 114 & 118 & 113 & 104 & 84 & 90 & 84 & 82 \\
\hline ORS & TF-TRH & 89 & 97 & 103 & 97 & 72 & 89 & 81 & 74 \\
\hline SSP & TF-TRH & 62 & 63 & 65 & 58 & 72 & 89 & 79 & 64 \\
\hline EMPS & TF-TRH & 72 & 84 & 82 & 73 & 73 & 86 & 75 & 75 \\
\hline NVM & TF-TRH & 70 & 82 & 74 & 67 & 74 & 89 & 72 & 71 \\
\hline
\end{tabular}


Valores de PAM (mm Hg)

\begin{tabular}{|c|c|c|c|c|c|c|c|c|c|}
\hline & & \multicolumn{4}{|c|}{ SESSÃO PRÉ } & \multicolumn{4}{|c|}{ SESSÃO PÓS } \\
\hline Voluntárias & & REP & ED & OCLU & REC & REP & ED & OCLU & REC \\
\hline MARO & SED-PLA & 96 & 107 & 109 & 92 & 92 & 114 & 97 & 93 \\
\hline RCA & SED-PLA & 102 & 118 & 111 & 92 & 112 & 111 & 112 & 116 \\
\hline MYI & SED-PLA & 102 & 106 & 102 & 96 & 104 & 98 & 103 & 109 \\
\hline FSP & SED-PLA & 88 & 94 & 91 & 94 & 91 & 103 & 92 & 96 \\
\hline VLO & SED-PLA & 100 & 105 & 108 & 105 & 99 & 105 & 104 & 100 \\
\hline MRS & SED-PLA & 92 & 96 & 92 & 92 & 84 & 92 & 86 & 83 \\
\hline LMLM & SED-PLA & 80 & 95 & 81 & 80 & 78 & 93 & 84 & 85 \\
\hline MDTBS & SED-PLA & 86 & 97 & 88 & 83 & 101 & 92 & 92 & 85 \\
\hline MVL & SED-PLA & 86 & 92 & 92 & 89 & 95 & 91 & 101 & 90 \\
\hline POS & SED-TRH & 98 & 96 & 89 & 90 & 88 & 113 & 99 & 85 \\
\hline LMS & SED-TRH & 99 & 123 & 116 & 109 & 98 & 129 & 103 & 95 \\
\hline JJS & SED-TRH & 122 & 128 & 145 & 134 & 105 & 102 & 107 & 104 \\
\hline NPB & SED-TRH & 117 & 104 & 105 & 101 & 95 & 107 & 107 & 105 \\
\hline MLC & SED-TRH & 105 & 111 & 108 & 102 & 102 & 102 & 94 & 99 \\
\hline AAR & SED-TRH & 94 & 95 & 105 & 95 & 100 & 105 & 101 & 96 \\
\hline GG & SED-TRH & 84 & 91 & 87 & 85 & 91 & 90 & 83 & 95 \\
\hline MESS & SED-TRH & 115 & 118 & 117 & 108 & 100 & 114 & 113 & 105 \\
\hline SRGP & SED-TRH & 85 & 102 & 94 & 82 & 91 & 95 & 76 & 90 \\
\hline SRS & TF-PLA & 104 & 112 & 106 & 117 & 92 & 111 & 104 & 97 \\
\hline MCSA & TF-PLA & 125 & 126 & 113 & 114 & 118 & 114 & 117 & 108 \\
\hline ITS & TF-PLA & 105 & 96 & 107 & 105 & 95 & 98 & 106 & 95 \\
\hline SCS & TF-PLA & 84 & 88 & 82 & 86 & 90 & 93 & 85 & 86 \\
\hline WGF & TF-PLA & 114 & 121 & 116 & 113 & 106 & 121 & 113 & 113 \\
\hline JMBP & TF-PLA & 104 & 112 & 111 & 106 & 102 & 108 & 103 & 96 \\
\hline DMB & TF-PLA & 92 & 111 & 99 & 90 & 86 & 99 & 88 & 90 \\
\hline SMS & TF-TRH & 99 & 102 & 65 & 82 & 99 & 101 & 108 & 105 \\
\hline ALCLM & TF-TRH & 125 & 138 & 136 & 123 & 106 & 113 & 100 & 98 \\
\hline ORS & TF-TRH & 117 & 120 & 129 & 122 & 95 & 107 & 100 & 95 \\
\hline MAO & TF-TRH & 82 & 91 & 87 & 83 & 92 & 77 & 71 & 77 \\
\hline SSP & TF-TRH & 81 & 81 & 85 & 73 & 90 & 109 & 100 & 87 \\
\hline
\end{tabular}


Valores de FC (bpm)

\begin{tabular}{|c|c|c|c|c|c|c|c|c|c|}
\hline & & \multicolumn{4}{|c|}{ SESSÃO PRÉ } & \multicolumn{4}{|c|}{ SESSÃO PÓS } \\
\hline Voluntárias & & REP & ED & OCLU & REC & REP & ED & OCLU & REC \\
\hline MMC & SED-PLA & 73 & 80 & 71 & 74 & 74 & 78 & 69 & 72 \\
\hline MARO & SED-PLA & 64 & 76 & 73 & 72 & 68 & 79 & 69 & 69 \\
\hline RCA & SED-PLA & 70 & 80 & 75 & 74 & 75 & 81 & 78 & 73 \\
\hline MYI & SED-PLA & 66 & 69 & 65 & 65 & 72 & 75 & 74 & 79 \\
\hline FSP & SED-PLA & 78 & 86 & 81 & 78 & 71 & 78 & 77 & 74 \\
\hline VLO & SED-PLA & 67 & 69 & 68 & 68 & 66 & 67 & 68 & 68 \\
\hline MRS & SED-PLA & 67 & 76 & 74 & 69 & 60 & 66 & 63 & 61 \\
\hline LMLM & SED-PLA & 65 & 73 & 65 & 67 & 66 & 73 & 66 & 67 \\
\hline MDTBS & SED-PLA & 59 & 65 & 60 & 59 & 73 & 72 & 67 & 66 \\
\hline MVL & SED-PLA & 65 & 66 & 71 & 68 & 63 & 67 & 68 & 70 \\
\hline POS & SED-TRH & 55 & 60 & 57 & 56 & 54 & 63 & 57 & 55 \\
\hline LMS & SED-TRH & 50 & 63 & 55 & 50 & 54 & 68 & 56 & 52 \\
\hline JJS & SED-TRH & 55 & 70 & 58 & 65 & 70 & 71 & 58 & 58 \\
\hline NPB & SED-TRH & 54 & 58 & 58 & 60 & 59 & 67 & 63 & 61 \\
\hline RFS & SED-TRH & 99 & 95 & 97 & 97 & 105 & 101 & 97 & 92 \\
\hline AAR & SED-TRH & 65 & 74 & 69 & 66 & 62 & 65 & 61 & 61 \\
\hline SMDR & SED-TRH & 44 & 48 & 46 & 45 & 46 & 69 & 47 & 47 \\
\hline GG & SED-TRH & 56 & 62 & 58 & 57 & 51 & 59 & 49 & 51 \\
\hline MESS & SED-TRH & 78 & 82 & 78 & 73 & 63 & 68 & 59 & 63 \\
\hline SRGP & SED-TRH & 58 & 71 & 65 & 62 & 65 & 76 & 67 & 64 \\
\hline VFG & SED-TRH & 67 & 74 & 68 & 66 & 63 & 79 & 65 & 62 \\
\hline SRS & TF-PLA & 70 & 79 & 70 & 75 & 80 & 87 & 78 & 82 \\
\hline MCSA & TF-PLA & 69 & 73 & 69 & 72 & 73 & 77 & 82 & 77 \\
\hline ITS & TF-PLA & 77 & 79 & 73 & 74 & 66 & 73 & 69 & 69 \\
\hline SCS & TF-PLA & 59 & 62 & 63 & 61 & 57 & 65 & 61 & 59 \\
\hline WGF & TF-PLA & 62 & 74 & 58 & 63 & 64 & 72 & 54 & 60 \\
\hline DMB & TF-PLA & 61 & 83 & 67 & 60 & 55 & 63 & 55 & 56 \\
\hline SMS & TF-TRH & 48 & 56 & 49 & 47 & 54 & 59 & 59 & 55 \\
\hline ALCLM & TF-TRH & 63 & 76 & 69 & 65 & 63 & 68 & 65 & 63 \\
\hline ORS & TF-TRH & 65 & 72 & 67 & 66 & 64 & 74 & 63 & 63 \\
\hline NVM & TF-TRH & 58 & 75 & 61 & 59 & 57 & 74 & 56 & 55 \\
\hline
\end{tabular}


Valores de FSA [ml(min.100ml)]

\begin{tabular}{c|c|c|c|c|c|c|c|c|c}
\hline & & \multicolumn{3}{|c|}{ SESSÃO PRÉ } & \multicolumn{4}{c}{ SESSÃO PÓS } \\
\hline Voluntárias & Grupos & REP & ED & OCLU & REC & REP & ED & OCLU & REC \\
\hline MMC & SED-PLA & 1,5 & 1,6 & 1,4 & 1,3 & 0,9 & 1,2 & 1,8 & 0,8 \\
\hline MARO & SED-PLA & 2,5 & 3,8 & 3,9 & 2,8 & 1,0 & 1,4 & 1,1 & 1,2 \\
\hline RCA & SED-PLA & 1,7 & 2,0 & 1,8 & 1,8 & 2,6 & 2,4 & 1,6 & 2,2 \\
\hline MYI & SED-PLA & 1,4 & 1,4 & 1,5 & 1,1 & 1,3 & 1,2 & 0,7 & 0,0 \\
\hline FSP & SED-PLA & 3,5 & 5,2 & 5,8 & 4,4 & 2,6 & 3,4 & 2,6 & 2,1 \\
\hline VLO & SED-PLA & 0,8 & 0,5 & 0,4 & 0,4 & 2,5 & 2,1 & 1,1 & 0,9 \\
\hline LMLM & SED-PLA & 3,1 & 3,5 & 3,2 & 2,3 & 3,3 & 3,3 & 2,2 & 2,0 \\
\hline MVL & SED-PLA & 4,9 & 5,4 & 5,2 & 4,6 & 1,6 & 6,1 & 5,0 & 2,8 \\
\hline POS & SED-TRH & 4,0 & 4,4 & 4,3 & 4,5 & 1,5 & 2,2 & 2,4 & 1,4 \\
\hline AAR & SED-TRH & 2,2 & 3,1 & 3,1 & 2,6 & 2,7 & 2,8 & 2,2 & 1,8 \\
\hline SMDR & SED-TRH & 0,8 & 0,6 & 0,7 & 0,6 & 2,8 & 2,3 & 1,6 & 1,9 \\
\hline GG & SED-TRH & 1,4 & 1,9 & 1,3 & 1,1 & 2,7 & 2,7 & 2,0 & 1,7 \\
\hline SRS & TF-PLA & 2,6 & 2,6 & 3,0 & 2,8 & 2,4 & 2,9 & 1,8 & 2,1 \\
\hline MCSA & TF-PLA & 3,3 & 3,1 & 2,0 & 2,9 & 2,7 & 2,1 & 2,2 & 2,0 \\
\hline ITS & TF-PLA & 4,2 & 4,3 & 4,0 & 2,9 & 3,6 & 4,8 & 4,7 & 3,8 \\
\hline WGF & TF-PLA & 2,1 & 4,5 & 1,9 & 1,7 & 2,9 & 3,9 & 3,1 & 3,0 \\
\hline JMBP & TF-PLA & 1,5 & 2,5 & 1,9 & 1,4 & 1,8 & 4,2 & 2,6 & 5,0 \\
\hline SMS & TF-TRH & 1,1 & 1,6 & 1,3 & 0,9 & 0,7 & 0,9 & 0,8 & 0,7 \\
\hline ALCLM & TF-TRH & 1,6 & 1,8 & 2,0 & 1,7 & 2,3 & 2,6 & 2,3 & 2,1 \\
\hline SSP & TF-TRH & 5,0 & 5,5 & 5,0 & 4,2 & 3,2 & 5,0 & 4,2 & 5,9 \\
\hline EMPS & TF-TRH & 2,0 & 1,8 & 1,9 & 1,6 & 2,3 & 3,2 & 2,0 & 1,9 \\
\hline NVM & TF-TRH & 3,0 & 3,1 & 2,7 & 2,2 & 1,9 & 1,9 & 1,2 & 1,2 \\
\hline & & & & & & & & \\
\hline & & & & \\
\hline
\end{tabular}




\section{REFERÊNCIAS}

1. Guyton AC, Hall JE. Text Book of Medical Physilology. 10th ed. Philadelphia: Saunders Company; 2000.

2. Fonseca AM, Sauerbronn AVD, Bagnoli VR. Terapia de Reposição Hormonal. In Tratado de Ginecologia. 2ed. São Paulo; Medicas; 2002. p.626-40.

3. Rapkin AJ. Vasomotor symptoms in menopause: physiologic condition and central nervous system approaches to treatment. Am J Obstet Gynecol. 2007;196(2):97-106.

4. Lima GRB. Ginecologia Endócrina. In Síndrome do Climatério. Atheneu editora. São Paulo; 1995. p. 253-294.

5. Zarate A, Saucedo R, Basurto L, Martinez C. Cardiovascular disease as a current threat of older women. Relation to estrogens. Ginecol Obstet Mex. 2007;75(5):286-92.

6. Bentley-Lewis R, Koruda K, Seely EW. The metabolic syndrome in women. Nat Clin Pract Endocrinol Metab. 2007;3(10):696-704.

7. Brunette MG, Leclerc M. Effect of estrogen on calcium and sodium transport by the nephron luminal membranes. J Endocrinol . 2001;170(2):441-50.

8. Vongpatanasin $W$, Tuncel $M$, Mansour $Y$, Arbique $D$, Victor RG. Transdermal estrogen replacement therapy decreases sympathetic activity in postmenopausal women. Circulation. 2001;103(24):2903-8. 
9. Zanchetti A, Facchetti R, Cesana GC, Modena MG, Pirrelli A, Sega R. Menopause-related blood pressure increase and its relationship to age and body mass index: the SIMONA epidemiological study. $J$ Hypertens. 2005;23(12):2269-76.

10. Bagnoli VR, Fonseca AM; Assis JS; Rosas FC; Pinotti, JA. Terapia de reposição hormonal: esquemas. In: Bagnoli VR, Fonseca AM, Assis JA. Terapia de reposição hormonal em situações especiais. 1ed. São Paulo: Editora Revinter; 2001.

11. Shanafelt TD, Barton DL, Adjei AA, Loprinzi CL. Pathophysiology and treatment of hot flashes. Mayo Clin Proc. 2002;77(11):1207-18.

12. Rodstrom K, Bengtsson C, Lissner L, Milsom I, Sundh V, Bjorkelund C. A longitudinal study of the treatment of hot flushes: the population study of women in Gothenburg during a quarter of a century. Menopause. 2002;9(3):156-61.

13. The Board of Trustees of The North American Menopause Society (NAMS). Estrogen and progestogen use in peri-and postmenopausal women: March 2007 position statement of The North American Menopause Society. Menopause. 2007;14(2):168-182.

14. Utian WH, Shoupe D, Bachmann G, Pinkerton JV, Pickar JH. Relief of vasomotor symptoms and vaginal atrophy with lower doses of conjugated equine estrogens and medroxyprogesterone acetate. Fertil Steril. 2001;75(6):1065-79.

15. Stampfer MJ, Colditz GA, Willett WC, Manson JE, Rosner B, Speizer FE, Hennekens $\mathrm{CH}$. Postmenopausal estrogen therapy and cardiovascular disease. Ten-year follow-up from the nurses' health study. N Engl J Med. 1991;325(11):756-62. 
16. Hulley S, Grady D, Bush T, Furberg C, Herrington D, Riggs B, Vittinghoff E. Randomized trial of estrogen plus progestin for secondary prevention of coronary heart disease in postmenopausal women. Heart and Estrogen/progestin Replacement Study (HERS) Research Group. JAMA. 1998;280(7):605-13.

17. Skouby S. Consequenses for HRT following the HERS II and WHI reports: the primum non nocere is important, but translation into quo vadis is even more essential. Acta Obstet Gynecol Scand. 2002;81(9):793-8.

18. Khan MA, Heagerty AM, Kitchener $H$, McNamee $R$, Cherry NM, Hannaford P. Oestrogen and women's heart disease: ESPRIT-UK. QJM. 2000;93(10):699-700.

19. Viscoli CM, Brass LM, Kernan WN, Sarrel PM, Suissa S, Horwitz RI. A clinical trial of estrogen-replacement therapy after ischemic stroke. $\mathrm{N}$ Engl J Med. 2001;345(17):1243-9.

20. The Women's Health Initiative Investigators. Risks and benefits of estrogen plus progestins in healthy poostmenopausal women: principal results from the Women's Health Initiative randomized controlled trial. JAMA. 2002;288:321-333.

21. The Women's Health Initiative Investigators Steering Comitee. Effects of Conjugated Equine Estrogen in Postmenopausal Women with Histerectomy - The Women's Health Initiative Randomized Controlled Trial. JAMA 2004;291:1701-1712.

22. Mosca L, Appel LJ, Benjamin EJ, Berra K, Chandra-Strobos N, Fabunmi RP, et al. Evidence-based guidelines for cardiovascular disease prevention in women. American Heart Association scientific statement. Arterioscler Thromb Vasc Biol. 2004;24(3):e29-50. 
23. Million Women Study Collaborators. Breast cancer and hormonereplacement therapy in the Million Women Study. Lancet. 2003;362:419-427.

24. European Agency for Evaluation of Medical Products. EMEA Public Statmenton Recent Publications Regarding Hormone Replacement Therapy. EMEA;33065;03. Disponivel em: http://www.emea.europa.eu/pdfs/human/press/pus/3306503en.pdf.

25. Modena MG, Sismondi $\mathrm{P}$, Mueck AO, Kuttenn F, Lignieres $B$, Verhaeghe J, Foidart JM, Caufriez A, Genazzani AR. New evidence regarding hormone replacement therapies is urgently required transdermal postmenopausal hormone therapy differs from oral hormone therapy in risks and benefits. Maturitas 2005;52(1):1-10.

26. Jones PP, Snitker S, Skinner JS, Ravussin E. Gender differences in muscle sympathetic nerve activity: effect of body fat distribution. $A m \mathrm{~J}$ Physiol. 1996;270(2 Pt 1):E363-6.

27. Mark AL. The sympathetic nervous system in hypertension: a potential long-term regulator of arterial pressure. J Hypertens Suppl. 1996;14(5):S159-65.

28. Brook RD, Julius S. Autonomic imbalance, hypertension, and cardiovascular risk. Am J Hypertens. 2000;13(6 Pt 2):112S-122S.

29. Tinucci T, Abrahão SB, Mion Jr D. Sistema Nervoso Simpático na Hipertensão Arterial - Microneurografia. In: Cruz J, Barros RT, Sesso RCCC, Neto ED, Suassuna JHR, Heilberg IP, Gouveia Filho WL. Atualidades em Nefrologia. São Paulo: Sarvier; 1994. p. 289-298

30. Matsukawa T, Mano T, Gotoh E, Ishii M. Elevated sympathetic nerve activity in patients with accelerated essential hypertension. J Clin Invest. 1993;92(1):25-8. 
31. Floras JS, Hara K. Sympathoneural and haemodynamic characteristics of young subjects with mild essential hypertension. $J$ Hypertens. 1993;11(6):647-55.

32. Ng AV, Callister R, Johnson DG, Seals DR. Age and gender influence muscle sympathetic nerve activity at rest in healthy humans. Hypertension. 1993;21(4):498-503.

33. Rowe JW, Troen BR. Sympathetic nervous system and aging in man. Endocr Rev. 1980;1(2):167-79.

34. Matsukawa T, Sugiyama Y, Watanabe T, Kobayashi F, Mano T. Gender difference in age-related changes in muscle sympathetic nerve activity in healthy subjects. Am J Physiol. 1998;275(5 Pt 2):R1600-4.

35. Hunt BE, Taylor JA, Hamner JW, Gagnon M, Lipsitz LA. Estrogen replacement therapy improves baroreflex regulation of vascular sympathetic outflow in postmenopausal women. Circulation. 2001;103(24):2909-14.

36. Weitz G, Elam M, Born J, Fehm HL, Dodt C. Postmenopausal estrogen administration suppresses muscle sympathetic nerve activity. J Clin Endocrinol Metab. 2001;86(1):344-8.

37. With Chartbook on Trends in the Heath of Americans. National Center of Health Statistics. Disponível em http;//www.cdc.gov/nchs/hus.htm.

38. Akahoshi M, Soda M, Nakashima E, Shimaoka K, Seto S, Yano K. Effects of menopause on trends of serum cholesterol, blood pressure, and body mass index. Circulation. 1996;94(1):61-6.

39. Mikkola TS, Clarkson TB. Estrogen replacement therapy, atherosclerosis, and vascular function. Cardiovasc Res. 2002;53(3):605-19. 
40. Butkevich A, Abraham C, Phillips RA. Hormone replacement therapy and 24-hour blood pressure profile of postmenopausal women. Am J Hypertens. 2000;13(9):1039-41.

41. Cacciatore B, Paakkari I, Hasselblatt R, Nieminen MS, Toivonen J, Tikkanen MI, Ylikorkala O. Randomized comparison between orally and transdermally administered hormone replacement therapy regimens of long-term effects on 24-hour ambulatory blood pressure in postmenopausal women. Am J Obstet Gynecol. 2001;184(5):904-9.

42. Utian WH. Effect of postmenopausal estrogen therapy on diastolic blood pressure and bodyweight. Maturitas. 1978;1(1):3-8.

43. Wren BG, Routledge DA. Blood pressure changes: oestrogens in climacteric women. Med J Aust. 1981;2(10):528-31.

44. Pripp U, Hall G, Csemiczky G, Eksborg S, Landgren BM, SchenckGustafsson K. A randomized trial on effects of hormone therapy on ambulatory blood pressure and lipoprotein levels in women with coronary artery disease. J Hypertens. 1999;17(10):1379-86.

45. The Writing Group for the PEPI Trial. Effects of Estrogen or Estrogen/Progestin Regimens on Heart Disease Risk factors in Postmenopausal Women - The Postmenopausal Estrogen/Progestin Interventions (PEPI) Trial. JAMA. 1995;273:199-208.

46. Schunkert H, Danser AH, Hense HW, Derkx FH, Kurzinger S, Riegger GA. Effects of estrogen replacement therapy on the renin-angiotensin system in postmenopausal women. Circulation. 1997;95(1):39-45.

47. Maturana MA, Irigoyen MC, Spritzer PM. Menopause, estrogens, and endothelial dysfunction: current concepts. Clinics. 2007;62(1):77-86.

48. Khalil RA. Sex hormones as potential modulators of vascular function in hypertension. Hypertension. 2005;46(2):249-54. 
49. Hermenegildo C, Oviedo PJ, Cano A. Cyclooxygenases regulation by estradiol on endothelium. Curr Pharm Des. 2006;12(2):205-15.

50. Kesim MD, Aydin Y, Erdemir M, Atis A. Nitric oxide in postmenopausal women taking three different HRT regimens. Maturitas. 2005;50(1):527.

51. Nilsson S, Gustafsson JA. Estrogen receptor transcription and transactivation: Basic aspects of estrogen action. Breast Cancer Res. 2000;2(5):360-6.

52. Pate RR, Pratt M, Blair SN, Haskell WL, Macera CA, Bouchard C, Ettinger W, Heath GW, King AC et al. Physical activity and public health. A recommendation from the Centers for Disease Control and Prevention and the American College of Sports Medicine. JAMA. 1995;273(5):402-7.

53. American College of Sports Medicine Position Stand. The Recommended Quantity and Quality of Exercise for Developing and Maintaining Cardiorespiratory and Muscular Fitness, and Flexibility in Healthy Adults. Med. Sci. Sports Exerc. 1998;30(6):975-991.

54. Forjaz CL, Cardoso CG, Jr., Rezk CC, Santaella DF, Tinucci T. Postexercise hypotension and hemodynamics: the role of exercise intensity. J Sports Med Phys Fitness. 2004;44(1):54-62.

55. Hamer M. The anti-hypertensive effects of exercise: integrating acute and chronic mechanisms. Sports Med. 2006;36(2):109-16.

56. Ray CA, Hume KM. Sympathetic neural adaptations to exercise training in humans: insights from microneurography. Med Sci Sports Exerc. 1998;30(3):387-91.

57. Asikainen TM, Kukkonen-Harjula K, Miilunpalo S. Exercise for health for early postmenopausal women: a systematic review of randomised controlled trials. Sports Med. 2004;34(11):753-78. 
58. Moriyama CK, Oneda B, Bernardo FR, Cardoso CG, Jr., Forjaz CL, Abrahao SB, et al. A randomized, placebo-controlled trial of the effects of physical exercises and estrogen therapy on health-related quality of life in postmenopausal women. Menopause. 2008;15(4 Pt 1):613-8.

59. Manson JE, Hu FB, Rich-Edwards JW, Colditz GA, Stampfer MJ, Willett WC, Speizer FE, Hennekens $\mathrm{CH}$. A prospective study of walking as compared with vigorous exercise in the prevention of coronary heart disease in women. N Engl J Med. 1999;341(9):650-8.

60. Lee IM, Rexrode KM, Cook NR, Manson JE, Buring JE. Physical activity and coronary heart disease in women: is "no pain, no gain" passe? JAMA. 2001;285(11):1447-54.

61. Asikainen TM, Miilunpalo S, Kukkonen-Harjula $K$, Nenonen A, Pasanen M, Rinne M, Uusi-rasi K, Oja P, Vuori I. Walking trials in postmenopausal women: effect of low doses of exercise and exercise fractionization on coronary risk factors. Scand J Med Sci Sports. 2003;13(5):284-92.

62. Hamdorf PA, Withers RT, Penhall RK, Haslam MV. Physical training effects on the fitness and habitual activity patterns of elderly women. Arch Phys Med Rehabil. 1992;73(7):603-8.

63. Ready AE, Naimark B, Ducas J, Sawatzky JV, Boreskie SL, Drinkwater DT, Oosterveen S. Influence of walking volume on health benefits in women post-menopause. Med Sci Sports Exerc. 1996;28(9):1097-105.

64. Lindheim SR, Notelovitz M, Feldman EB, Larsen S, Khan FY, Lobo RA. The independent effects of exercise and estrogen on lipids and lipoproteins in postmenopausal women. Obstet Gynecol. 1994;83(2):167-72. 
65. Katyal S, Freeman M, Miller JA, Thomas SG. Short-term aerobic training and circulatory function in women: age and hormonereplacement therapy. Clin Sci. 2003;104(3):267-73.

66. Snabes MC, Herd JA, Schuyler N, Dunn K, Spence DW, Young RL. In normal postmenopausal women physiologic estrogen replacement therapy fails to improve exercise tolerance: a randomized, doubleblind, placebo-controlled, crossover trial. Am J Obstet Gynecol. 1996;175(1):110-4.

67. Kirwan LD, Mertens DJ, Kavanagh T, Thomas SG, Goodman JM. Exercise training in women with heart disease: influence of hormone replacement therapy. Med Sci Sports Exerc. 2003;35(2):185-92.

68. Oneda B, Forjaz CL, Bernardo FR, Araujo TG, Gusmao JL, Labes E, Abrahão SB, Mion Jr D, Fonseca AM, Tinucci T. Low-dose estrogen therapy does not change postexercise hypotension, sympathetic nerve activity reduction, and vasodilation in healthy postmenopausal women. Am J Physiol Heart Circ Physiol. 2008;295(4):H1802-8.

69. Expert Committee on the Diagnosis and Classification of Diabetes Mellitus. Report of the expert committee on the diagnosis and classification of diabetes mellitus. Diabetes Care. 2003;26(Suppl 1 ):S5-20.

70. Executive Summary of The Third Report of The National Cholesterol Education Program (NCEP) Expert Panel on Detection, Evaluation, And Treatment of High Blood Cholesterol In Adults (Adult Treatment Panel III). JAMA. 2001;285:2486-97.

71. Chobanian AV, Bakris GL, Black HR, Cushman WC, Green LA, Izzo Jr JL, Jones DW, Materson BJ, Oparil S, Roccella EJ. The Seventh Report of the Joint National Committee on Prevention, Detection, Evaluation, and Treatment of High Blood Pressure: the JNC 7 report. JAM. 2003;289(19):2560-72. 
72. Bhambhani $\mathrm{Y}$, Singh $\mathrm{M}$. Ventilatory thresholds during a graded exercise test. Respiration. 1985;47(2):120-8.

73. Scherrer U, Pryor SL, Bertocci LA, Victor RG. Arterial baroreflex buffering of sympathetic activation during exercise-induced elevations in arterial pressure. J Clin Invest. 1990;86(6):1855-61.

74. Mano GM, Souza VF, Pierin AM, Lima JC, Ignes EC, Ortega KC, Mion Jr D. Assessment of the DIXTAL DX-2710 automated oscillometric device for blood pressure measurement with the validation protocols of the British Hypertension Society (BHS) and the Association for the Advancement of Medical Instrumentation (AAMI). Arq Bras Cardiol 2002;79(6):606-10, 601-5.

75. Siggaard-Andersen J. Venous occlusion plethysmography on the calf. Evaluation of diagnosis and results in vascular surgery. Dan Med Bull. 1970;17:Suppl I:1-68.

76. Wallin BG, Fagius J. Peripheral sympathetic neural activity in conscious humans. Annu Rev Physiol. 1988;50:565-76.

77. Anderson EA, Mark A.L. . Microneurographic measurements of sympathetic nerve activity in humans. In: Schneiderman N, Weiss SM, Kaufmann PG. Handbook of research methods in cardiovascular behavioral medicine: Plenum Publishing Corporation; 1989. p. 107115.

78. Hagbarth KE. Exteroceptive, proprioceptive, and sympathetic activity recorded with microelectrodes from human peripheral nerves. Mayo Clin Proc. 1979;54(6):353-65.

79. Grassi G, Seravalle G, Calhoun DA, Mancia G. Physical training and baroreceptor control of sympathetic nerve activity in humans. Hypertension. 1994;23(3):294-301. 
80. Ueno LM, Drager LF, Rodrigues AC, Rondon MU, Braga AM, Mathias W, Krieger E, Barreto AC, Middlekauff HR, Lorenzi-Filho G, Negrão CE. Effects of exercise training in patients with chronic heart failure and sleep apnea. Sleep. 2009;32(5):637-47.

81. Ray CA. Sympathetic adaptations to one-legged training. J App/ Physiol. 1999;86(5):1583-7.

82. Ray CA, Carrasco DI. Isometric handgrip training reduces arterial pressure at rest without changes in sympathetic nerve activity. $A m J$ Physiol Heart Circ Physiol. 2000;279(1):H245-9.

83. Duncan JJ, Farr JE, Upton SJ, Hagan RD, Oglesby ME, Blair SN. The effects of aerobic exercise on plasma catecholamines and blood pressure in patients with mild essential hypertension. JAMA. 1985;254(18):2609-13.

84. Pagani M, Somers V, Furlan R, Dell'Orto S, Conway J, Baselli G, Cerutti S, Sleight $\mathrm{P}$, Malliani $\mathrm{A}$. Changes in autonomic regulation induced by physical training in mild hypertension. Hypertension. 1988;12(6):600-10.

85. Wang PH, Horng HC, Cheng MH, Chao HT, Chao KC. Standard and low-dose hormone therapy for postmenopausal women--focus on the breast. Taiwan J Obstet Gynecol. 2007;46(2):127-34.

86. O'Sullivan AJ, Crampton LJ, Freund J, Ho KK. The route of estrogen replacement therapy confers divergent effects on substrate oxidation and body composition in postmenopausal women. J Clin Invest. 1998;102(5):1035-40.

87. Duanmu Z, Lapanowski K, Dunbar JC. Insulin-like growth factor-I decreases sympathetic nerve activity: the effect is modulated by glycemic status. Proc Soc Exp Biol Med. 1997;216(1):93-7. 
88. Sverrisdottir YB, Elam M, Herlitz H, Bengtsson BA, Johannsson G. Intense sympathetic nerve activity in adults with hypopituitarism and untreated growth hormone deficiency. $J$ Clin Endocrinol Metab. $1998 ; 83(6): 1881-5$.

89. Pescatello LS, Franklin BA, Fagard R, Farquhar WB, Kelley GA, Ray CA. American College of Sports Medicine position stand. Exercise and hypertension. Med Sci Sports Exerc. 2004;36(3):533-53.

90. Dickhuth HH, Lehmann M, Auch-Schwelk W, Meinertz T, Keul J. Physical training, vegetative regulation, and cardiac hypertrophy. $J$ Cardiovasc Pharmacol. 1987;10 Suppl 6:S71-8.

91. Cardoso Jr CG. Efeito da terapia hormonal oral de estrogênio e do treinamento aeróbico sobre a sensibilidade à insulina e as respostas hemodinâmicas e autonômicas à hiperinsulinemia aguda em mulheres na pós-menopausa [tese]. São Paulo: Universidade de São Paulo; 2009.

92. Rosano GM, Patrizi R, Leonardo F, Ponikowski P, Collins P, Sarrel PM, Chierchia SL. Effect of estrogen replacement therapy on heart rate variability and heart rate in healthy postmenopausal women. Am J Cardiol. 1997;80(6):815-7.

93. Christ M, Seyffart K, Wehling M. Attenuation of heart-rate variability in postmenopausal women on progestin-containing hormone replacement therapy. Lancet. 1999;353(9168):1939-40.

94. Mercuro G, Zoncu S, Piano D, Pilia I, Lao A, Melis GB,Cherchi A. Estradiol-17beta reduces blood pressure and restores the normal amplitude of the circadian blood pressure rhythm in postmenopausal hypertension. Am J Hypertens. 1998;11(8 Pt 1):909-13. 
95. Seccareccia F, Menotti A. Physical activity, physical fitness and mortality in a sample of middle aged men followed-up 25 years. $J$ Sports Med Phys Fitness. 1992;32(2):206-13.

96. Harvey PJ, Morris BL, Kubo T, Picton PE, Su WS, Notarius CF, Floras JS. Hemodynamic after-effects of acute dynamic exercise in sedentary normotensive postmenopausal women. J Hypertens. 2005;23(2):28592.

97. Amaral SL, Zorn TM, Michelini LC. Exercise training normalizes wallto-lumen ratio of the gracilis muscle arterioles and reduces pressure in spontaneously hypertensive rats. J Hypertens. 2000;18(11):1563-72.

98. Arakawa K. Exercise, a measure to lower blood pressure and reduce other risks. Clin Exp Hypertens. 1999;21(5-6):797-803.

99. Collins P. Vascular effects of hormones. Maturitas 2001;38(1):45-50.

100. Czarnecka D, Kawecka-Jaszcz K, Olszanecka A, Dembinska-Kiec A, Malczewska-Malec M, Zdzienicka A, Guevara I. The effect of hormone replacement therapy on endothelial function in postmenopausal women with hypertension. Med Sci Monit. 2004;10(2):CR55-61.

101. Smolders RG, van der Mooren MJ, Kenemans P, van der Linden PW, Stehouwer CD, Sipkema P. 17 beta-Estradiol induces a rapid, endothelium-dependent, sex-specific vasodilatation in spontaneous constricted rat arterioles. Am J Obstet Gynecol. 2002;187(2):375-81.

102. Kirwan LD, MacLusky NJ, Shapiro HM, Abramson BL, Thomas SG, Goodman JM. Acute and chronic effects of hormone replacement therapy on the cardiovascular system in healthy postmenopausal women. J Clin Endocrinol Metab. 2004;89(4):1618-29.

103. Vitale C, Mercuro G, Cerquetani E, Marazzi G, Patrizi R, Pelliccia F, Volterrani M, Fini M, Collins P, Rosano GM. Time since menopause 
influences the acute and chronic effect of estrogens on endothelial function. Arterioscler Thromb Vasc Biol. 2008;28(2):348-52.

104. Fink GR, Adams L, Watson JD, Innes JA, Wuyam B, Kobayashi I, Corfield DR, Murphy K, Jones T, Frackowiak RS et al. Hyperpnoea during and immediately after exercise in man: evidence of motor cortical involvement. J Physiol. 1995;489 ( Pt 3):663-75.

105. Mark AL, Victor RG, Nerhed C, Wallin BG. Microneurographic studies of the mechanisms of sympathetic nerve responses to static exercise in humans. Circ Res. 1985;57(3):461-9.

106. Victor RG, Seals DR, Mark AL. Differential control of heart rate and sympathetic nerve activity during dynamic exercise. Insight from intraneural recordings in humans. J Clin Invest 1987;79(2):508-16.

107. Seals DR, Chase PB, Taylor JA. Autonomic mediation of the pressor responses to isometric exercise in humans. J Appl Physiol. 1988;64(5):2190-6.

108. Seals DR. Sympathetic neural discharge and vascular resistance during exercise in humans. J Appl Physiol. 1989;66(5):2472-8.

109. Victor RG, Seals DR. Reflex stimulation of sympathetic outflow during rhythmic exercise in humans. Am J Physiol. 1989;257(6 Pt 2):H201724.

110. Seals DR. Sympathetic neural adjustments to stress in physically trained and untrained humans. Hypertension. 1991;17(1):36-43.

111. Ettinger SM, Silber DH, Gray KS, Smith MB, Yang QX, Kunselman $A R$, Sinoway LI. Effects of the ovarian cycle on sympathetic neural outflow during static exercise. J Appl Physiol. 1998;85(6):2075-81. 
112. Sanchez J, Sebert P. Sex differences in cardiac responses to breath holding during dynamic and isometric exercises. Eur J Appl Physiol Occup Physiol. 1983;50(3):429-44.

113. Haissly JC, Messin R, Degre S, Vandermoten P, Demaret B, Denolin $\mathrm{H}$. Comparative response to isometric (static) and dynamic exercise tests in coronary disease. Am J Cardiol. 1974;33(6):791-6.

114. Chapman JH, Elliott PW. Cardiovascular effects of static and dynamic exercise. Eur J Appl Physiol Occup Physiol. 1988;58(1-2):152-7.

115. Markel TA, Daley JC, 3rd, Hogeman CS, Herr MD, Khan MH, Gray KS, Kunselman AR, Sinoway LI. Aging and the exercise pressor reflex in humans. Circulation. 2003;107(5):675-8.

116. Wilson LB, Dyke CK, Parsons D, Wall PT, Pawelczyk JA, Williams RS, Mitchell $\mathrm{JH}$. Effect of skeletal muscle fiber type on the pressor response evoked by static contraction in rabbits. J Appl Physiol. 1995;79(5):1744-52.

117. Thomas GD, Sander M, Lau KS, Huang PL, Stull JT, Victor RG. Impaired metabolic modulation of alpha-adrenergic vasoconstriction in dystrophin-deficient skeletal muscle. Proc Natl Acad Sci U S A. 1998;95(25):15090-5.

118. Thomas GD, Victor RG. Nitric oxide mediates contraction-induced attenuation of sympathetic vasoconstriction in rat skeletal muscle. $J$ Physiol. 1998;506 ( Pt 3):817-26.

119. Fadel PJ, Wang Z, Watanabe H, Arbique D, Vongpatanasin W, Thomas GD. Augmented sympathetic vasoconstriction in exercising forearms of postmenopausal women is reversed by oestrogen therapy. J Physiol. 2004;561(Pt 3):893-901. 
120. Remensnyder JP, Mitchell JH, Sarnoff SJ. Functional sympatholysis during muscular activity. Observations on influence of carotid sinus on oxygen uptake. Circ Res. 1962;11:370-80.

121. Koch DW, Leuenberger UA, Proctor DN. Augmented leg vasoconstriction in dynamically exercising older men during acute sympathetic stimulation. J Physiol. 2003;551(Pt 1):337-44.

122. Du XJ, Dart AM, Riemersma RA. Sex differences in the parasympathetic nerve control of rat heart. Clin Exp Pharmacol Physiol. 1994;21(6):485-93.

123. Liu CC, Kuo TB, Yang CC. Effects of estrogen on gender-related autonomic differences in humans. Am J Physiol Heart Circ Physiol. 2003;285(5):H2188-93.

124. Ettinger SM, Silber DH, Collins BG, Gray KS, Sutliff G, Whisler SK, McClain JM, Smith MB, Yang QX, Sinoway LI. Influences of gender on sympathetic nerve responses to static exercise. J Appl Physiol. 1996;80(1):245-51.

125. Hayes SG, Moya Del Pino NB, Kaufman MP. Estrogen attenuates the cardiovascular and ventilatory responses to central command in cats. J Appl Physio.I 2002;92(4):1635-41.

126. Schmitt PM, Kaufman MP. High concentrations of 17beta -estradiol attenuate the exercise pressor reflex in male cats. J Appl Physiol. 2003;94(4):1431-6.

127. Schmitt PM, Kaufman MP. Estrogen attenuates the exercise pressor reflex in female cats. J Appl Physiol. 2003;95(4):1418-24.

128. Sudhir K, Elser MD, Jennings GL, Komesaroff PA. Estrogen supplementation decreases norepinephrine-induced vasoconstriction and total body norepinephrine spillover in perimenopausal women. Hypertension. 1997;30(6):1538-43. 
129. Schmitt PM, Kaufman MP. Estrogen's attenuating effect on the exercise pressor reflex is more opioid dependent in gonadally intact than in ovariectomized female cats. J Appl Physiol. 2005;98(2):633-9.

130. MCardle WD, Katch F, Katch V. Fisiologia do Exercício - Energia, Nutrição e Desempenho Humano. Rio de Janeiro: 4 ed. Guanabara Koogan; 1996. 